\title{
Minimal Length Scale Scenarios for Quantum Gravity
}

\author{
Sabine Hossenfelder \\ Nordita \\ Roslagstullsbacken 23 \\ 10691 Stockholm \\ Sweden \\ email: hossi@nordita.org
}

Accepted: 11 October 2012

Published: 29 January 2013

\begin{abstract}
We review the question of whether the fundamental laws of nature limit our ability to probe arbitrarily short distances. First, we examine what insights can be gained from thought experiments for probes of shortest distances, and summarize what can be learned from different approaches to a theory of quantum gravity. Then we discuss some models that have been developed to implement a minimal length scale in quantum mechanics and quantum field theory. These models have entered the literature as the generalized uncertainty principle or the modified dispersion relation, and have allowed the study of the effects of a minimal length scale in quantum mechanics, quantum electrodynamics, thermodynamics, black-hole physics and cosmology. Finally, we touch upon the question of ways to circumvent the manifestation of a minimal length scale in short-distance physics.
\end{abstract}

Keywords: minimal length, quantum gravity, generalized uncertainty principle 


\section{Imprint / Terms of Use}

Living Reviews in Relativity is a peer reviewed open access journal published by the Max Planck Institute for Gravitational Physics, Am Mühlenberg 1, 14476 Potsdam, Germany. ISSN 1433-8351.

This review is licensed under a Creative Commons Attribution-Non-Commercial 3.0 Germany License: http://creativecommons.org/licenses/by-nc/3.0/de/. Figures that have been previously published elsewhere may not be reproduced without consent of the original copyright holders.

Because a Living Reviews article can evolve over time, we recommend to cite the article as follows:

Sabine Hossenfelder,

"Minimal Length Scale Scenarios for Quantum Gravity",

Living Rev. Relativity, 16, (2013), 2. URL (accessed < date>):

http://www.livingreviews.org/lrr-2013-2

The date given as $<$ date $>$ then uniquely identifies the version of the article you are referring to.

\section{Article Revisions}

Living Reviews supports two ways of keeping its articles up-to-date:

Fast-track revision A fast-track revision provides the author with the opportunity to add short notices of current research results, trends and developments, or important publications to the article. A fast-track revision is refereed by the responsible subject editor. If an article has undergone a fast-track revision, a summary of changes will be listed here.

Major update A major update will include substantial changes and additions and is subject to full external refereeing. It is published with a new publication number.

For detailed documentation of an article's evolution, please refer to the history document of the article's online version at http://www. livingreviews.org/lrr-2013-2. 


\section{Contents}

1 Introduction $\quad 5$

2 A Minimal History $r$

3 Motivations 11

3.1 Thought experiments . . . . . . . . . . . . . . . . . . . 11

3.1.1 The Heisenberg microscope with Newtonian gravity . . . . . . . . . . . 11

3.1.2 The general relativistic Heisenberg microscope . . . . . . . . . . . . 13

3.1.3 Limit to distance measurements . . . . . . . . . . . . . . . . . . 16

3.1.4 Limit to clock synchronization . . . . . . . . . . . . . . . 17

3.1.5 Limit to the measurement of the black-hole-horizon area . . . . . . . . . . 18

3.1.6 A device-independent limit for non-relativistic particles . . . . . . . . . 19

3.1.7 Limits on the measurement of spacetime volumes . . . . . . . . . . . . . 21

3.2 String theory . . . . . . . . . . . . . . . . . . . . . 22

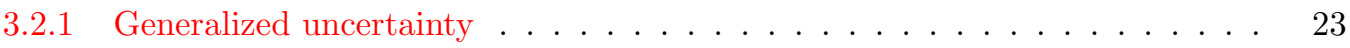

3.2.2 Spacetime uncertainty . . . . . . . . . . . . . . . . . . . . . . . . . . .

3.2.3 Taking into account Dp-Branes . . . . . . . . . . . . . . . . . . . . . . . . . . . . .

3.2 .4 T-duality . . . . . . . . . . . . . . . . . . . 30

3.3 Loop Quantum Gravity and Loop Quantum Cosmology . . . . . . . . . . . . . . . 32

3.4 Quantized conformal fluctuations . . . . . . . . . . . . . . . . . . . . . . . . . . . . . .

3.5 Asymptotically Safe Gravity . . . . . . . . . . . . . . . . . . . . . . . . . 37

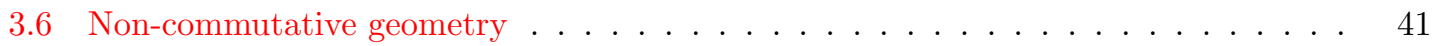

3.7 Miscellaneous . . . . . . . . . . . . . . . . . . . . . . . . . 44

3.8 Summary of motivations . . . . . . . . . . . . . . . . . . . . 45

4 Models and Applications $\quad 46$

4.1 Interpretation of a minimal length scale . . . . . . . . . . . . . . . . . . 46

4.2 Modified commutation relations . . . . . . . . . . . . . . . . 47

4.2.1 Recovering the minimal length from modified commutation relations . . . . 47

4.2 .2 The Snyder basis . . . . . . . . . . . . . . . . . . . 51

4.2 .3 The choice of basis in phase space . . . . . . . . . . . . . 53

4.2 .4 Multi-particle states . . . . . . . . . . . . . . . . . . 55

4.2 .5 Open problems . . . . . . . . . . . . . . . . 57

4.3 Quantum mechanics with a minimal length scale . . . . . . . . . . . . . . 58

4.3.1 Maximal localization states . . . . . . . . . . . . . . . 59

4.3.2 The Schrödinger equation with potential . . . . . . . . . . . . . 59

4.3.3 The Klein-Gordon and Dirac equation . . . . . . . . . . . . . . 60

4.4 Quantum field theory with a minimal length scale . . . . . . . . . . . . . . . . . 60

4.5 Deformed Special Relativity . . . . . . . . . . . . . . . . . . . . 61

4.6 Composite systems and statistical mechanics . . . . . . . . . . . . . . . . 62

4.7 Path-integral duality . . . . . . . . . . . . . . . . . . . . . 63 63

4.8 Direct applications of the uncertainty principle . . . . . . . . . . . . . . . 64

4.9 Miscellaneous . . . . . . . . . . . . . . . . . . . . 64

5 Discussion $\quad \mathbf{6 6}$

5.1 Interrelations . . . . . . . . . . . . . . . . . . . . . . . . . . . . . . . . . . 66

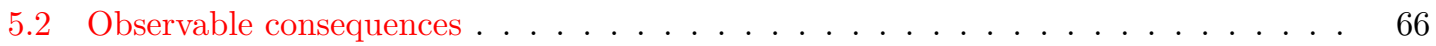

5.3 Is it possible that there is no minimal length? . . . . . . . . . . . . . . . 67 
7 Acknowledgements $\quad \mathbf{7 0}$

$\begin{array}{lr}\text { Index } & 71\end{array}$

$\begin{array}{lr}\text { References } & 72\end{array}$

\section{List of Tables}

$1 \quad$ Analogy between scales involved in D-particle scattering and the hydrogen atom. . 30 


\section{Introduction}

In the 5th century B.C., Democritus postulated the existence of smallest objects that all matter is built from and called them 'atoms'. In Greek, the prefix ' $a$ ' means 'not' and the word 'tomos' means 'cut'. Thus, atomos or atom means uncuttable or indivisible. According to Democritus' theory of atomism, "Nothing exists except atoms and empty space, everything else is opinion." Though variable in shape, Democritus' atoms were the hypothetical fundamental constituents of matter, the elementary building blocks of all that exists, the smallest possible entities. They were conjectured to be of finite size, but homogeneous and without substructure. They were the first envisioned end of reductionism.

2500 years later, we know that Democritus was right in that solids and liquids are composed of smaller entities with universal properties that are called atoms in his honor. But these atoms turned out to be divisible. And stripped of its electrons, the atomic nucleus too was found to be a composite of smaller particles, neutrons and protons. Looking closer still, we have found that even neutrons and protons have a substructure of quarks and gluons. At present, the standard model of particle physics with three generations of quarks and fermions and the vector fields associated to the gauge groups are the most fundamental constituents of matter that we know.

Like a Russian doll, reality has so far revealed one after another layer on smaller and smaller scales. This begs the question: Will we continue to look closer into the structure of matter, and possibly find more layers? Or is there a fundamental limit to this search, a limit beyond which we cannot go? And if so, is this a limit in principle or one in practice?

Any answer to this question has to include not only the structure of matter, but the structure of space and time itself, and therefore it has to include gravity. For one, this is because Democritus' search for the most fundamental constituents carries over to space and time too. Are space and time fundamental, or are they just good approximations that emerge from a more fundamental concept in the limits that we have tested so far? Is spacetime made of something else? Are there 'atoms' of space? And second, testing short distances requires focusing large energies in small volumes, and when energy densities increase, one finally cannot neglect anymore the curvature of the background.

In this review we will study this old question of whether there is a fundamental limit to the resolution of structures beyond which we cannot discover anything more. In Section 3, we will summarize different approaches to this question, and how they connect with our search for a theory of quantum gravity. We will see that almost all such approaches lead us to find that the possible resolution of structures is finite or, more graphically, that nature features a minimal length scale - though we will also see that the expression 'minimal length scale' can have different interpretations. While we will not go into many of the details of the presently pursued candidate theories for quantum gravity, we will learn what some of them have to say about the question. After the motivations, we will in Section 4 briefly review some approaches that investigate the consequences of a minimal length scale in quantum mechanics and quantum field theory, models that have flourished into one of the best motivated and best developed areas of the phenomenology of quantum gravity.

In the following, we use the unit convention $c=\hbar=1$, so that the Planck length $l_{\mathrm{Pl}}$ is the inverse of the Planck mass $m_{\mathrm{Pl}}=1 / l_{\mathrm{Pl}}$, and Newton's constant $G=l_{\mathrm{Pl}}^{2}=1 / m_{\mathrm{Pl}}^{2}$. The signature of the metric is $(1,-1,-1,-1)$. Small Greek indices run from 0 to 3, large Latin indices from 0 to 4, and small Latin indices from 1 to 3, except for Section 3.2, where small Greek indices run from 0 to $D$, and small Latin indices run from 2 to $D$. An arrow denotes the spatial component of a vector, for example $\vec{a}=\left(a_{1}, a_{2}, a_{3}\right)$. Bold-faced quantities are tensors in an index-free notation that will be used in the text for better readability, for example $\mathbf{p}=\left(p_{0}, p_{1}, p_{2}, p_{3}\right)$. Acronyms and abbreviations can be found in the index.

We begin with a brief historical background. 


\section{A Minimal History}

Special relativity and quantum mechanics are characterized by two universal constants, the speed of light, $c$, and Planck's constant, $\hbar$. Yet, from these constants alone one cannot construct either a constant of dimension length or mass. Though, if one had either, they could be converted into each other by use of $\hbar$ and $c$. But in 1899, Max Planck pointed out that adding Newton's constant $G$ to the universal constants $c$ and $\hbar$ allows one to construct units of mass, length and time [265]:

$$
\begin{aligned}
t_{\mathrm{Pl}} & \approx 10^{-43} \mathrm{~s} \\
l_{\mathrm{Pl}} & \approx 10^{-33} \mathrm{~cm} \\
m_{\mathrm{Pl}} & \approx 1.2 \times 10^{19} \mathrm{GeV} .
\end{aligned}
$$

Today these are known as the Planck time, Planck length and Planck mass, respectively. As we will see later, they mark the scale at which quantum effects of the gravitational interaction are expected to become important. But back in Planck's days their relevance was their universality, because they are constructed entirely from fundamental constants.

The idea of a minimal length was predated by that of the "chronon," a smallest unit of time, proposed by Robert Lévi [200] in 1927 in his "Hyphothèse de l'atome de temps" (hypothesis of time atoms), that was further developed by Pokrowski in the years following Lévi's proposal [266]. But that there might be limits to the divisibility of space and time remained a far-fetched speculation on the fringes of a community rapidly pushing forward the development of general relativity and quantum mechanics. It was not until special relativity and quantum mechanics were joined in the framework of quantum field theory that the possible existence of a minimal length scale rose to the awareness of the community.

With the advent of quantum field theory in the 1930s, it was widely believed that a fundamental length was necessary to cure troublesome divergences. The most commonly used regularization was a cut-off or some other dimensionful quantity to render integrals finite. It seemed natural to think of this pragmatic cut-off as having fundamental significance, an interpretation that however inevitably caused problems with Lorentz invariance, since the cut-off would not be independent of the frame of reference. Heisenberg was among the first to consider a fundamentally-discrete spacetime that would yield a cut-off, laid out in his letters to Bohr and Pauli. The idea of a fundamentally finite length or a maximum frequency was in these years studied by many, including Flint [110], March [219], Möglich [234] and Goudsmit [267], just to mention a few. They all had in common that they considered the fundamental length to be in the realm of subatomic physics on the order of the femtometer $\left(10^{-15} \mathrm{~m}\right)$.

The one exception was a young Russian, Matvei Bronstein. Today recognized as the first to comprehend the problem of quantizing gravity [138], Bronstein was decades ahead of his time. Already in 1936, he argued that gravity is in one important way fundamentally different from electrodynamics: Gravity does not allow an arbitrarily high concentration of charge in a small region of spacetime, since the gravitational 'charge' is energy and, if concentrated too much, will collapse to a black hole. Using the weak field approximation of gravity, he concluded that this leads to an inevitable limit to the precision of which one can measure the strength of the gravitational field (in terms of the Christoffel symbols).

In his 1936 article "Quantentheorie schwacher Gravitationsfelder" (Quantum theory of weak gravitational fields), Bronstein wrote [138, 70]:

"[T]he gravitational radius of the test-body $\left(G \rho V / c^{2}\right)$ used for the measurements should by no means be larger than its linear dimensions $\left(V^{1 / 3}\right)$; from this one obtains an upper bound for its density $\left(\rho \lesssim c^{2} / G V^{2 / 3}\right)$. Thus, the possibilities for measurements in this region are even more restricted than one concludes from the quantum-mechanical commutation relations. Without a profound change of the classical notions it therefore 
seems hardly possible to extend the quantum theory of gravitation to this region." ${ }^{1}$ $([70], \text { p. } 150)^{2}$

Few people took note of Bronstein's argument and, unfortunately, the history of this promising young physicist ended in a Leningrad prison in February 1938, where Matvei Bronstein was executed at the age of 31 .

Heisenberg meanwhile continued in his attempt to make sense of the notion of a fundamental minimal length of nuclear dimensions. In 1938, Heisenberg wrote "Über die in der Theorie der Elementarteilchen auftretende universelle Länge" (On the universal length appearing in the theory of elementary particles) [148], in which he argued that this fundamental length, which he denoted $r_{0}$, should appear somewhere not too far beyond the classical electron radius (of the order $100 \mathrm{fm}$ ).

This idea seems curious today, and has to be put into perspective. Heisenberg was very worried about the non-renormalizability of Fermi's theory of $\beta$-decay. He had previously shown [147] that applying Fermi's theory to the high center-of-mass energies of some hundred GeV lead to an 'explosion,' by which he referred to events of very high multiplicity. Heisenberg argued this would explain the observed cosmic ray showers, whose large number of secondary particles we know today are created by cascades (a possibility that was discussed already at the time of Heisenberg's writing, but not agreed upon). We also know today that what Heisenberg actually discovered is that Fermi's theory breaks down at such high energies, and the four-fermion coupling has to be replaced by the exchange of a gauge boson in the electroweak interaction. But in the 1930s neither the strong nor the electroweak force was known. Heisenberg then connected the problem of regularization with the breakdown of the perturbation expansion of Fermi's theory, and argued that the presence of the alleged explosions would prohibit the resolution of finer structures:

"If the explosions actually exist and represent the processes characteristic for the constant $r_{0}$, then they maybe convey a first, still unclear, understanding of the obscure properties connected with the constant $r_{0}$. These should certainly express themselves in difficulties of measurements with a precision better than $r_{0} \ldots$ The explosions would have the effect... that measurements of positions are not possible to a precision better than $r_{0} .{ }^{3}$ ([148], p. 31)

In hindsight we know that Heisenberg was, correctly, arguing that the theory of elementary particles known in the 1930s was incomplete. The strong interaction was missing and Fermi's theory indeed non-renormalizable, but not fundamental. Today we also know that the standard model of particle physics is renormalizable and know techniques to deal with divergent integrals that do not necessitate cut-offs, such as dimensional regularization. But lacking that knowledge, it is understandable that Heisenberg argued that taking into account gravity was irrelevant for the existence of a fundamental length:

"The fact that [the Planck length] is significantly smaller than $r_{0}$ makes it valid to leave aside the obscure properties of the description of nature due to gravity, since they at least in atomic physics - are totally negligible relative to the much coarser obscure

1 “[D]er Gravitationsradius des zur Messung dienenden Probekörpers $\left(G \rho V / c^{2}\right)$ soll keineswegs größer als seine linearen Abmessungen $\left(V^{1 / 3}\right)$ sein; daraus entsteht eine obere Grenze für seine Dichte $\left(\rho \lesssim c^{2} / G V^{2 / 3}\right)$. Die Messungsmöglichkeiten sind also in dem Gebiet noch mehr beschränkt als es sich aus den quantenmechanischen Vertauschungsrelationen schliessen läßt. Ohne eine tiefgreifende Änderung der klassischen Begriffe, scheint es daher kaum möglich, die Quantentheorie der Gravitation auch auf dieses Gebiet auszudehnen."

2 Translations from German to English: SH.

3 "Wenn die Explosionen tatsächlich existieren und die für die Konstante $r_{0}$ eigentlich charakeristischen Prozesse darstellen, so vermitteln sie vielleicht ein erstes, noch unklares Verständnis der unanschaulichen Züge, die mit der Konstanten $r_{0}$ verbunden sind. Diese sollten sich ja wohl zunächst darin äußern, daß die Messung einer den Wert $r_{0}$ unterschreitenden Genauigkeit zu Schwierigkeiten führt... [D]ie Explosionen [würden] dafür sorgen..., daß Ortsmessungen mit einer $r_{0}$ unterschreitenden Genauigkeit unmöglich sind." 
properties that go back to the universal constant $r_{0}$. For this reason, it seems hardly possible to integrate electric and gravitational phenomena into the rest of physics until the problems connected to the length $r_{0}$ are solved." 4 ([148], p. 26)

Heisenberg apparently put great hope in the notion of a fundamental length to move forward the understanding of elementary matter. In 1939 he expressed his belief that a quantum theory with a minimal length scale would be able to account for the discrete mass spectrum of the (then known) elementary particles [149]. However, the theory of quantum electrodynamics was developed to maturity, the 'explosions' were satisfactorily explained and, without being hindered by the appearance of any fundamentally finite resolution, experiments probed shorter and shorter scales. The divergences in quantum field theory became better understood and discrete approaches to space and time remained unappealing due to their problems with Lorentz invariance.

In a 1947 letter to Heisenberg, Pauli commented on the idea of a smallest length that Heisenberg still held dearly and explained his reservations, concluding "Extremely put, I would not be surprised if your 'universal' length turned out to be a mere figment of imagination." [254]. (For more about Heisenberg's historical involvement with the universal length, the interested reader is referred to Kragh's very recommendable article [199].)

In 1930, in a letter to his student Rudolf Peierls [150], Heisenberg mentioned that he was trying to make sense of a minimal length by letting the position operators be non-commuting $\left[\hat{x}^{\nu}, \hat{x}^{\mu}\right] \neq 0$. He expressed his hope that Peierls ask Pauli how to proceed with this idea:

"So far, I have not been able to make mathematical sense of such commutation relations... Do you or Pauli have anything to say about the mathematical meaning of such commutation relations?" 5 ([150], p. 16)

But it took 17 years until Snyder, in 1947, made mathematical sense of Heisenberg's idea. ${ }^{6}$ Snyder, who felt that that the use of a cut-off in momentum space was a "distasteful arbitrary procedure" [288], worked out a modification of the canonical commutation relations of position and momentum operators. In that way, spacetime became Lorentz-covariantly non-commutative, but the modification of commutation relations increased the Heisenberg uncertainty, such that a smallest possible resolution of structures was introduced (a consequence Snyder did not explicitly mention in his paper). Though Snyder's approach was criticized for the difficulties of inclusion of translations [316], it has received a lot of attention as the first to show that a minimal length scale need not be in conflict with Lorentz invariance.

In 1960, Peres and Rosen [262] studied uncertainties in the measurement of the average values of Christoffel symbols due to the impossibility of concentrating a mass to a region smaller than its Schwarzschild radius, and came to the same conclusion as Bronstein already had, in 1936,

"The existence of these quantum uncertainties in the gravitational field is a strong argument for the necessity of quantizing it. It is very likely that a quantum theory of gravitation would then generalize these uncertainty relations to all other Christoffel symbols." ([262], p. 336)

4 "Der Umstand, daß [die Plancklänge] wesentlich kleiner ist als $r_{0}$, gibt uns das Recht, von den durch die Gravitation bedingten unanschaulichen Zügen der Naturbeschreibung zunächst abzusehen, da sie - wenigstens in der Atomphysik - völlig untergehen in den viel gröberen unanschaulichen Zügen, die von der universellen Konstanten $r_{0}$ herrühren. Es dürfte aus diesen Gründen wohl kaum möglich sein, die elektrischen und die Gravitationserscheinungen in die übrige Physik einzuordnen, bevor die mit der Länge $r_{0}$ zusammenhängenden Probleme gelöst sind."

5 "Mir ist es bisher nicht gelungen, solchen Vertauschungs-Relationen einen vernünftigen mathematischen Sinn zuzuordnen...Fällt Ihnen oder Pauli nicht vielleicht etwas über den mathematischen Sinn solcher VertauschungsRelationen ein?"

6 The story has been told [313] that Peierls asked Pauli, Pauli passed the question on to his colleague Oppenheimer, who asked his student Hartland Snyder. However, in a 1946 letter to Pauli [289], Snyder encloses his paper without any mention of it being an answer to a question posed to him by others. 
While they considered the limitations for measuring the gravitational field itself, they did not study the limitations these uncertainties induce on the ability to measure distances in general.

It was not until 1964, that Mead pointed out the peculiar role that gravity plays in our attempts to test physics at short distances [222, 223]. He showed, in a series of thought experiments that we will discuss in Section 3.1, that this influence does have the effect of amplifying Heisenberg's measurement uncertainty, making it impossible to measure distances to a precision better than Planck's length. And, since gravity couples universally, this is, though usually negligible, an inescapable influence on all our experiments.

Mead's work did not originally attain a lot of attention. Decades later, he submitted his recollection [224] that "Planck's proposal that the Planck mass, length, and time should form a fundamental system of units... was still considered heretical well into the 1960s," and that his argument for the fundamental relevance of the Planck length met strong resistance:

"At the time, I read many referee reports on my papers and discussed the matter with every theoretical physicist who was willing to listen; nobody that I contacted recognized the connection with the Planck proposal, and few took seriously the idea of [the Planck length] as a possible fundamental length. The view was nearly unanimous, not just that I had failed to prove my result, but that the Planck length could never play a fundamental role in physics. A minority held that there could be no fundamental length at all, but most were then convinced that a [different] fundamental length..., of the order of the proton Compton wavelength, was the wave of the future. Moreover, the people I contacted seemed to treat this much longer fundamental length as established fact, not speculation, despite the lack of actual evidence for it." ([224], p. 15)

But then in the mid 1970s then Hawking's calculation of a black hole's thermodynamical properties [145] introduced the 'transplanckian problem.' Due to the, in principle infinite, blue shift of photons approaching a black-hole horizon, modes with energies exceeding the Planck scale had to be taken into account to calculate the emission rate. A great many physicists have significantly advanced our understanding of black-hole physics and the Planck scale, too many to be named here. However, the prominent role played by John Wheeler, whose contributions, though not directly on the topic of a minimal length, has connected black-hole physics with spacetime foam and the Planckian limit, and by this inspired much of what followed.

Unruh suggested in 1995 [308] that one use a modified dispersion relation to deal with the difficulty of transplanckian modes, so that a smallest possible wavelength takes care of the contributions beyond the Planck scale. A similar problem exists in inflationary cosmology [220] since tracing back in time small frequencies increases the frequency till it eventually might surpass the Planck scale at which point we no longer know how to make sense of general relativity. Thus, this issue of transplanckian modes in cosmology brought up another reason to reconsider the possibility of a minimal length or a maximal frequency, but this time the maximal frequency was at the Planck scale rather than at the nuclear scale. Therefore, it was proposed $[180,144]$ that this problem too might be cured by implementing a minimum length uncertainty principle into inflationary cosmology.

Almost at the same time, Majid and Ruegg [213] proposed a modification for the commutators of spacetime coordinates, similar to that of Snyder, following from a generalization of the Poincaré algebra to a Hopf algebra, which became known as $\kappa$-Poincaré. Kempf et al. [175, 174, 184, 178] developed the mathematical basis of quantum mechanics that took into account a minimal length scale and ventured towards quantum field theory. There are by now many variants of models employing modifications of the canonical commutation relations in order to accommodate a minimal length scale, not all of which make use of the complete $\kappa$-Poincaré framework, as will be discussed later in Sections 4.2 and 4.5. Some of these approaches were shown to give rise to a modification of the dispersion relation, though the physical interpretation and relevance, as well 
as the phenomenological consequences of this relation are still under debate.

In parallel to this, developments in string theory revealed the impossibility of resolving arbitrarily small structures with an object of finite extension. It had already been shown in the late 1980s $[140,10,9,11,310]$ that string scattering in the super-Planckian regime would result in a generalized uncertainty principle, preventing a localization to better than the string scale (more on this in Section 3.2). In 1996, John Schwarz gave a talk at SLAC about the generalized uncertainty principles resulting from string theory and thereby inspired the 1999 work by Adler and Santiago [3] who almost exactly reproduced Mead's earlier argument, apparently without being aware of Mead's work. This picture was later refined when it became understood that string theory not only contains strings but also higher dimensional objects, known as branes, which will be discussed in Section 3.2.

In the following years, a generalized uncertainty principle and quantum mechanics with the Planck length as a minimal length received an increasing amount of attention as potential cures for the transplanckian problem, a natural UV-regulator, and as possible manifestations of a fundamental property of quantum spacetime. In the late 1990s, it was also noted that it is compatible with string theory to have large or warped extra dimensions that can effectively lower the Planck scale into the $\mathrm{TeV}$ range. With this, the fundamental length scale also moved into the reach of collider physics, resulting in a flurry of activity. ${ }^{7}$

Today, how to resolve the apparent disagreements between the quantum field theories of the standard model and general relativity is one of the big open questions in theoretical physics. It is not that we cannot quantize gravity, but that the attempt to do so leads to a perturbatively nonrenormalizable and thus fundamentally nonsensical theory. The basic reason is that the coupling constant of gravity, Newton's constant, is dimensionful. This leads to the necessity to introduce an infinite number of counter-terms, eventually rendering the theory incapable of prediction.

But the same is true for Fermi's theory that Heisenberg was so worried about that he argued for a finite resolution where the theory breaks down, and mistakenly so, since he was merely pushing an effective theory beyond its limits. So we have to ask then if we might be making the same mistake as Heisenberg, in that we falsely interpret the failure of general relativity to extend beyond the Planck scale as the occurrence of a fundamentally finite resolution of structures, rather than just the limit beyond which we have to look for a new theory that will allow us to resolve smaller distances still?

If it was only the extension of classical gravity, laid out in many thought experiments that will be discussed in Section 3.1, that had us believing the Planck length is of fundamental importance, then the above historical lesson should caution us we might be on the wrong track. Yet, the situation today is different from the one that Heisenberg faced. Rather than pushing a quantum theory beyond its limits, we are pushing a classical theory and conclude that its short-distance behavior is troublesome, which we hope to resolve with quantizing the theory. And, as we will see, several attempts at a UV-completion of gravity, discussed in Sections 3.2-3.7, suggest that the role of the Planck length as a minimal length carries over into the quantum regime as a dimensionful regulator, though in very different ways, feeding our hopes that we are working on unveiling the last and final Russian doll.

For a more exhaustive coverage of the history of the minimal length, the interested reader is referred to [141].

7 Though the hope of a lowered Planck scale pushing quantum gravitational effects into the reach of the Large Hadron Collider seems, at the time of writing, to not have been fulfilled. 


\section{Motivations}

\subsection{Thought experiments}

Thought experiments have played an important role in the history of physics as the poor theoretician's way to test the limits of a theory. This poverty might be an actual one of lacking experimental equipment, or it might be one of practical impossibility. Luckily, technological advances sometimes turn thought experiments into real experiments, as was the case with Einstein, Podolsky and Rosen's 1935 paradox. But even if an experiment is not experimentally realizable in the near future, thought experiments serve two important purposes. First, by allowing the thinker to test ranges of parameter space that are inaccessible to experiment, they may reveal inconsistencies or paradoxes and thereby open doors to an improvement in the fundamentals of the theory. The complete evaporation of a black hole and the question of information loss in that process is a good example for this. Second, thought experiments tie the theory to reality by the necessity to investigate in detail what constitutes a measurable entity. The thought experiments discussed in the following are examples of this.

\subsubsection{The Heisenberg microscope with Newtonian gravity}

Let us first recall Heisenberg's microscope, that lead to the uncertainty principle [146]. Consider a photon with frequency $\omega$ moving in direction $x$, which scatters on a particle whose position on the $x$-axis we want to measure. The scattered photons that reach the lens of the microscope have to lie within an angle $\epsilon$ to produce an image from which we want to infer the position of the particle (see Figure 1). According to classical optics, the wavelength of the photon sets a limit to the possible resolution $\Delta x$

$$
\Delta x \gtrsim \frac{1}{2 \pi \omega \sin \epsilon} .
$$

But the photon used to measure the position of the particle has a recoil when it scatters and transfers a momentum to the particle. Since one does not know the direction of the photon to better than $\epsilon$, this results in an uncertainty for the momentum of the particle in direction $x$

$$
\Delta p_{x} \gtrsim \omega \sin \epsilon .
$$

Taken together one obtains Heisenberg's uncertainty (up to a factor of order one)

$$
\Delta x \Delta p_{x} \gtrsim \frac{1}{2 \pi} .
$$

We know today that Heisenberg's uncertainty is not just a peculiarity of a measurement method but much more than that - it is a fundamental property of the quantum nature of matter. It does not, strictly speaking, even make sense to consider the position and momentum of the particle at the same time. Consequently, instead of speaking about the photon scattering off the particle as if that would happen in one particular point, we should speak of the photon having a strong interaction with the particle in some region of size $R$.

Now we will include gravity in the picture, following the treatment of Mead [222]. For any interaction to take place and subsequent measurement to be possible, the time elapsed between the interaction and measurement has to be at least on the order of the time, $\tau$, the photon needs to travel the distance $R$, so that $\tau \gtrsim R$. The photon carries an energy that, though in general tiny, exerts a gravitational pull on the particle whose position we wish to measure. The gravitational acceleration acting on the particle is at least on the order of

$$
a \approx \frac{G \omega}{R^{2}},
$$




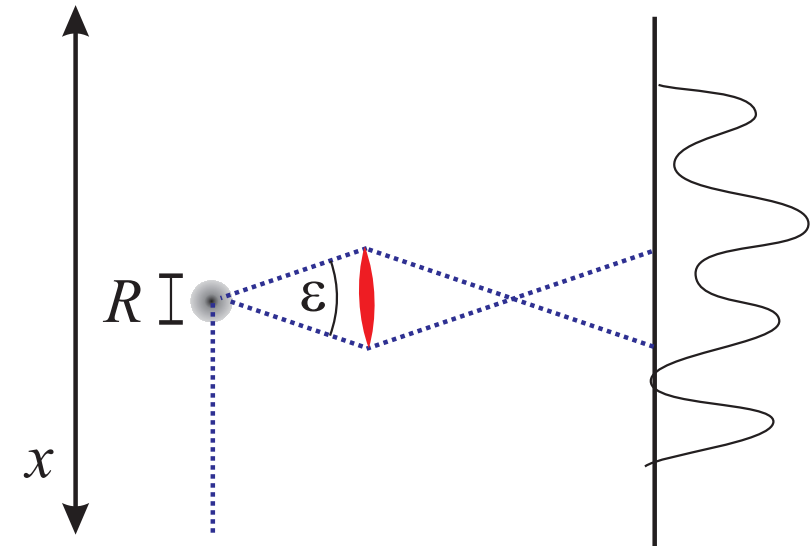

Figure 1: Heisenberg's microscope. A photon moving along the $x$-axis scatters off a probe within an interaction region of radius $R$ and is detected by a microscope (indicated by a lens and screen) with opening angle $\epsilon$.

and, assuming that the particle is non-relativistic and much slower than the photon, the acceleration lasts about the duration the photon is in the region of strong interaction. From this, the particle acquires a velocity of $v \approx a R$, or

$$
v \approx \frac{G \omega}{R} .
$$

Thus, in the time $R$, the acquired velocity allows the particle to travel a distance of

$$
L \approx G \omega .
$$

However, since the direction of the photon was unknown to within the angle $\epsilon$, the direction of the acceleration and the motion of the particle is also unknown. Projection on the $x$-axis then yields the additional uncertainty of

$$
\Delta x \gtrsim G \omega \sin \epsilon
$$

Combining (8) with (2), one obtains

$$
\Delta x \gtrsim \sqrt{G}=l_{\mathrm{Pl}} .
$$

One can refine this argument by taking into account that strictly speaking during the measurement, the momentum of the photon, $\omega$, increases by $G m \omega / R$, where $m$ is the mass of the particle. This increases the uncertainty in the particle's momentum (3) to

$$
\Delta p_{x} \gtrsim \omega\left(1+\frac{G m}{R}\right) \sin \epsilon,
$$

and, for the time the photon is in the interaction region, translates into a position uncertainty $\Delta x \approx R \Delta p / m$

$$
\Delta x \gtrsim \omega\left(\frac{R}{m}+G\right) \sin \epsilon,
$$

which is larger than the previously found uncertainty (8) and thus (9) still follows. 
Adler and Santiago [3] offer pretty much the same argument, but add that the particle's momentum uncertainty $\Delta p$ should be on the order of the photon's momentum $\omega$. Then one finds

$$
\Delta x \gtrsim G \Delta p .
$$

Assuming that the normal uncertainty and the gravitational uncertainties add linearly, one arrives at

$$
\Delta x \gtrsim \frac{1}{\Delta p}+G \Delta p .
$$

Any uncertainty principle with a modification of this or similar form has become known in the literature as 'generalized uncertainty principle' (GUP). Adler and Santiago's work was inspired by the appearance of such an uncertainty principle in string theory, which we will investigate in Section 3.2. Adler and Santiago make the interesting observation that the GUP (13) is invariant under the replacement

$$
l_{\mathrm{Pl}} \Delta p \leftrightarrow \frac{1}{l_{\mathrm{Pl}} \Delta p},
$$

which relates long to short distances and high to low energies.

These limitations, refinements of which we will discuss in the following Sections 3.1.2-3.1.7, apply to the possible spatial resolution in a microscope-like measurement. At the high energies necessary to reach the Planckian limit, the scattering is unlikely to be elastic, but the same considerations apply to inelastic scattering events. Heisenberg's microscope revealed a fundamental limit that is a consequence of the non-commutativity of position and momentum operators in quantum mechanics. The question that the GUP then raises is what modification of quantum mechanics would give rise to the generalized uncertainty, a question we will return to in Section 4.2.

Another related argument has been put forward by Scardigli [275], who employs the idea that once one arrives at energies of about the Planck mass and concentrates them to within a volume of radius of the Planck length, one creates tiny black holes, which subsequently evaporate. This effects scales in the same way as the one discussed here, and one arrives again at (13).

\subsubsection{The general relativistic Heisenberg microscope}

The above result makes use of Newtonian gravity, and has to be refined when one takes into account general relativity. Before we look into the details, let us start with a heuristic but instructive argument. One of the most general features of general relativity is the formation of black holes under certain circumstances, roughly speaking when the energy density in some region of spacetime becomes too high. Once matter becomes very dense, its gravitational pull leads to a total collapse that ends in the formation of a horizon. ${ }^{8}$ It is usually assumed that the Hoop conjecture holds [306]: If an amount of energy $\omega$ is compacted at any time into a region whose circumference in every direction is $R \leq 4 \pi G \omega$, then the region will eventually develop into a black hole. The Hoop conjecture is unproven, but we know from both analytical and numerical studies that it holds to very good precision $[107,168]$.

Consider now that we have a particle of energy $\omega$. Its extension $R$ has to be larger than the Compton wavelength associated to the energy, so $R \geq 1 / \omega$. Thus, the larger the energy, the better the particle can be focused. On the other hand, if the extension drops below $4 \pi G E$, then a black hole is formed with radius $2 \omega G$. The important point to notice here is that the extension of the black hole grows linearly with the energy, and therefore one can achieve a minimal possible extension, which is on the order of $R \sim \sqrt{G}$.

\footnotetext{
8 In the classical theory, inside the horizon lies a singularity. This singularity is expected to be avoided in quantum gravity, but how that works or doesn't work is not relevant in the following.
} 
For the more detailed argument, we follow Mead [222] with the general relativistic version of the Heisenberg microscope that was discussed in Section 3.1.1. Again, we have a particle whose position we want to measure by help of a test particle. The test particle has a momentum vector $(\omega, \vec{k})$, and for completeness we consider a particle with rest mass $\mu$, though we will see later that the tightest constraints come from the limit $\mu \rightarrow 0$.

The velocity $v$ of the test particle is

$$
v=\frac{k}{\sqrt{\mu^{2}+k^{2}}},
$$

where $k^{2}=\omega^{2}-\mu^{2}$, and $k=|\vec{k}|$. As before, the test particle moves in the $x$ direction. The task is now to compute the gravitational field of the test particle and the motion it causes on the measured particle.

To obtain the metric that the test particle creates, we first change into the rest frame of the particle by boosting into $x$-direction. Denoting the new coordinates with primes, the measured particle moves towards the test particle in direction $-x^{\prime}$, and the metric is a Schwarzschild metric. We will only need it on the $x$-axis where we have $y=z=0$, and thus

$$
g_{00}^{\prime}=1+2 \phi^{\prime}, \quad g_{11}^{\prime}=-\frac{1}{g_{00}^{\prime}}, \quad g_{22}^{\prime}=g_{33}^{\prime}=-1
$$

where

$$
\phi^{\prime}=\frac{G \mu}{\left|x^{\prime}\right|},
$$

and the remaining components of the metric vanish. Using the transformation law for tensors

$$
g_{\mu \nu}=\frac{\partial\left(x^{\prime}\right)^{\kappa}}{\partial x^{\mu}} \frac{\partial\left(x^{\prime}\right)^{\alpha}}{\partial x^{\nu}} g_{\kappa \alpha}^{\prime}
$$

with the notation $x^{0}=t, x^{1}=x, x^{2}=y, x^{3}=z$, and the same for the primed coordinates, the Lorentz boost from the primed to unprimed coordinates yields in the rest frame of the measured particle

$$
\begin{aligned}
& g_{00}=\frac{1+2 \phi}{1+2 \phi\left(1-v^{2}\right)}+2 \phi, \quad g_{11}=-\frac{-1+2 \phi v^{2}}{1+2 \phi\left(1-v^{2}\right)}+2 v^{2} \phi \\
& g_{01}=g_{10}=-\frac{2 v \phi}{1+\phi\left(1-v^{2}\right)}-2 v \phi, \quad g_{22}^{\prime}=g_{33}^{\prime}=-1,
\end{aligned}
$$

where

$$
\phi=\frac{\phi^{\prime}}{1-v^{2}}=-\frac{G \omega}{R}
$$

Here, $R=v t-x$ is the mean distance between the test particle and the measured particle. To avoid a horizon in the rest frame, we must have $2 \phi^{\prime}<1$, and thus from Eq. (21)

$$
-2 \phi^{\prime}=2 \frac{G \omega}{R}\left(1-v^{2}\right)<1 .
$$

Because of Eq. (2), $\Delta x \geq 1 / \omega$ but also $\Delta x \geq R$, which is the area in which the particle may scatter, thus

$$
\Delta x^{2} \gtrsim \frac{R}{\omega} \gtrsim 2 G\left(1-v^{2}\right)
$$


We see from this that, as long as $v^{2} \ll 1$, the previously found lower bound on the spatial resolution $\Delta x$ can already be read off here, and we turn our attention towards the case where $1-v^{2} \ll 1$. From (21) we see that this means we work in the limit where $-\phi \gg 1$.

To proceed, we need to estimate now how much the measured particle moves due to the test particle's vicinity. For this, we note that the world line of the measured particle must be timelike. We denote the velocity in the $x$-direction with $u$, then we need

$$
d s^{2}=\left(g_{00}+2 g_{10} u+g_{11} u^{2}\right) \mathrm{d} t^{2} \geq 0 .
$$

Now we insert Eq. (20) and follow Mead [222] by introducing the abbreviation

$$
\alpha=1+2 \phi\left(1-v^{2}\right) .
$$

Because of Eq. (22), $0<\alpha<1$. We simplify the requirement of Eq. (24) by leaving $u^{2}$ alone on the left side of the inequality, subtracting 1 and dividing by $u-1$. Taking into account that $\phi \leq 0$ and $v \leq 1$, one finds after some algebra

$$
u \geq \frac{1+2 \phi(1+\alpha)}{1-2 \phi v^{2}(1+\alpha)},
$$

and

$$
\frac{u}{1-u} \geq-\frac{1}{2}(1+2 \phi) .
$$

One arrives at this estimate with reduced effort if one makes it clear to oneself what we want to estimate. We want to know, as previously, how much the particle, whose position we are trying to measure, will move due to the gravitational attraction of the particle we are using for the measurement. The faster the particles pass by each other, the shorter the interaction time and, all other things being equal, the less the particle we want to measure will move. Thus, if we consider a photon with $v=1$, we are dealing with the case with the least influence, and if we find a minimal length in this case, it should be there for all cases. Setting $v=1$, one obtains the inequality Eq. (27) with greatly reduced work.

Now we can continue as before in the non-relativistic case. The time $\tau$ required for the test particle to move a distance $R$ away from the measured particle is at least $\tau \gtrsim R /(1-u)$, and during this time the measured particle moves a distance

$$
L=u \tau \gtrsim R \frac{u}{1-u} \gtrsim \frac{R}{2}(-1-2 \phi) .
$$

Since we work in the limit $-\phi \gg 1$, this means

$$
L \gtrsim G \omega,
$$

and projection on the $x$-axis yields as before (compare to Eq. (8)) for the uncertainty added to the measured particle because the photon's direction was known only to precision $\epsilon$

$$
\Delta x \gtrsim G \omega \sin \epsilon .
$$

This combines with (2), to again give

$$
\Delta x \gtrsim l_{\mathrm{Pl}} .
$$

Adler and Santiago [3] found the same result by using the linear approximation of Einstein's field equation for a cylindrical source with length $l$ and radius $\rho$ of comparable size, filled by a 
radiation field with total energy $\omega$, and moving in the $x$ direction. With cylindrical coordinates $x, r, \phi$, the line element takes the form [3]

$$
\mathrm{d} s^{2}=\mathrm{d} t^{2}-\mathrm{d} x^{2}-\mathrm{d} y^{2}-\mathrm{d} z^{2}+f(r, x, t)(\mathrm{dt}-\mathrm{d} x)^{2},
$$

where the function $f$ is given by

$$
\begin{aligned}
f(r, x, t) & =\frac{4 G \omega}{l} g(r) \theta(x-t) \theta(t-x-l) \\
g(r) & = \begin{cases}r^{2} / \rho^{2} & \text { for } r<\rho \\
1+\ln \left(r^{2} / \rho^{2}\right) & \text { for } r>\rho\end{cases}
\end{aligned}
$$

In this background, one can then compute the motion of the measured particle by using the Newtonian limit of the geodesic equation, provided the particle remains non-relativistic. In the longitudinal direction, along the motion of the test particle one finds

$$
\frac{\mathrm{d}^{2} x}{\mathrm{~d} t^{2}}=\frac{1}{2} \frac{\partial f}{\partial x} .
$$

The derivative of $f$ gives two delta-functions at the front and back of the cylinder with equal momentum transfer but of opposite direction. The change in velocity to the measured particle is

$$
\Delta \dot{x}=2 G \frac{\omega}{l} g(r) .
$$

Near the cylinder $g(r)$ is of order one, and in the time of passage $\tau \sim l$, the particle thus moves approximately

$$
2 G \omega
$$

which is, up to a factor of 2, the same result as Mead's (29). We note that Adler and Santiago's argument does not make use of the requirement that no black hole should be formed, but that the appropriateness of the non-relativistic and weak-field limit is questionable.

\subsubsection{Limit to distance measurements}

Wigner and Salecker [274] proposed the following thought experiment to show that the precision of length measurements is limited. Consider that we try to measure a length by help of a clock that detects photons, which are reflected by a mirror at distance $D$ and return to the clock. Knowing the speed of light is universal, from the travel-time of the photon we can then extract the distance it has traveled. How precisely can we measure the distance in this way?

Consider that at emission of the photon, we know the position of the (non-relativistic) clock to precision $\Delta x$. This means, according to the Heisenberg uncertainty principle, we cannot know its velocity to better than

$$
\Delta v=\frac{1}{2 M \Delta x}
$$

where $M$ is the mass of the clock. During the time $T=2 D$ that the photon needed to travel towards the mirror and back, the clock moves by $T \Delta v$, and so acquires an uncertainty in position of

$$
\Delta x+\frac{T}{2 M \Delta x},
$$


which bounds the accuracy by which we can determine the distance $D$. The minimal value that this uncertainty can take is found by varying with respect to $\Delta x$ and reads

$$
\Delta x_{\min }=\sqrt{\frac{T}{2 M}} .
$$

Taking into account that our measurement will not be causally connected to the rest of the world if it creates a black hole, we require $D>2 M G$ and thus

$$
\Delta x_{\min } \gtrsim l_{\mathrm{Pl}} .
$$

\subsubsection{Limit to clock synchronization}

From Mead's [222] investigation of the limit for the precision of distance measurements due to the gravitational force also follows a limit on the precision by which clocks can be synchronized.

We will consider the clock synchronization to be performed by the passing of light signals from some standard clock to the clock under question. Since the emission of a photon with energy spread $\Delta \omega$ by the usual Heisenberg uncertainty is uncertain by $\Delta T \sim 1 /(2 \Delta \omega)$, we have to take into account the same uncertainty for the synchronization.

The new ingredient comes again from the gravitational field of the photon, which interacts with the clock in a region $R$ over a time $\tau \gtrsim R$. If the clock (or the part of the clock that interacts with the photon) remains stationary, the (proper) time it records stands in relation to $\tau$ by $T=\tau \sqrt{g_{00}}$ with $g_{00}$ in the rest frame of the clock, given by Eq. (20), thus

$$
T=\tau \sqrt{1-\frac{4 G \omega}{r}} .
$$

Since the metric depends on the energy of the photon and this energy is not known precisely, the error on $\omega$ propagates into $T$ by

$$
(\Delta T)^{2}=\left(\frac{\partial T}{\partial \omega}\right)^{2}(\Delta \omega)^{2},
$$

thus

$$
\Delta T \sim \frac{2 G \tau}{r \sqrt{1-4 G \omega / r}} \Delta \omega .
$$

Since in the interaction region $\tau \gtrsim R \gtrsim r$, we can estimate

$$
\Delta T \gtrsim \frac{2 G}{\sqrt{1-4 G \omega / R}} \Delta \omega \gtrsim 2 G \Delta \omega .
$$

Multiplication of (45) with the normal uncertainty $\Delta T \gtrsim 1 /(2 \Delta \omega)$ yields

$$
\Delta T \gtrsim l_{\mathrm{Pl}} .
$$

So we see that the precision by which clocks can be synchronized is also bound by the Planck scale.

However, strictly speaking the clock does not remain stationary during the interaction, since it moves towards the photon due to the particles' mutual gravitational attraction. If the clock has a velocity $u$, then the proper time it records is more generally given by

$$
T=\int \mathrm{d} s \sim \tau \sqrt{g_{00}+2 g_{01} u+g_{11} u^{2}} .
$$


Using (20) and proceeding as before, one estimates the propagation of the error in the frequency by using $v=1$ and $u \leq 1$

$$
\left|\frac{\mathrm{d} T}{\mathrm{~d} \omega}\right| \gtrsim \tau \frac{8 G}{r} \frac{1}{\sqrt{1+4 G \omega / r}}
$$

and so with $\tau \gtrsim R \gtrsim r$

$$
\Delta T \gtrsim \tau \frac{G}{R} \Delta \omega \gtrsim G \Delta \omega
$$

Therefore, taking into account that the clock does not remain stationary, one still arrives at (46).

\subsubsection{Limit to the measurement of the black-hole-horizon area}

The above microscope experiment investigates how precisely one can measure the location of a particle, and finds the precision bounded by the inevitable formation of a black hole. However, this position uncertainty is for the location of the measured particle however and not for the size of the black hole or its radius. There is a simple argument why one would expect there to also be a limit to the precision by which the size of a black hole can be measured, first put forward in [91]. When the mass of a black-hole approaches the Planck mass, the horizon radius $R \sim G M$ associated to the mass becomes comparable to its Compton wavelength $\lambda=1 / M$. Then, quantum fluctuations in the position of the black hole should affect the definition of the horizon.

A somewhat more elaborate argument has been studied by Maggiore [208] by a thought experiment that makes use once again of Heisenberg's microscope. However, this time one wants to measure not the position of a particle, but the area of a (non-rotating) charged black hole's horizon. In Boyer-Lindquist coordinates, the horizon is located at the radius

$$
R_{H}=G M\left[1+\left(1-\frac{Q^{2}}{G M^{2}}\right)^{\frac{1}{2}}\right]
$$

where $Q$ is the charge and $M$ is the mass of the black hole.

To deduce the area of the black hole, we detect the black hole's Hawking radiation and aim at tracing it back to the emission point with the best possible accuracy. For the case of an extremal black hole $\left(Q^{2}=G M^{2}\right)$ the temperature is zero and we perturb the black hole by sending in photons from asymptotic infinity and wait for re-emission.

If the microscope detects a photon of some frequency $\omega$, it is subject to the usual uncertainty (2) arising from the photon's finite wavelength that limits our knowledge about the photon's origin. However, in addition, during the process of emission the mass of the black hole changes from $M+\omega$ to $M$, and the horizon radius, which we want to measure, has to change accordingly. If the energy of the photon is known only up to an uncertainty $\Delta p$, then the error propagates into the precision by which we can deduce the radius of the black hole

$$
\Delta R_{H} \sim\left|\frac{\partial R_{H}}{\partial M}\right| \Delta p
$$

With use of (50) and assuming that no naked singularities exist in nature $M^{2} G \leq Q^{2}$ one always finds that

$$
\Delta R_{H} \gtrsim 2 G \Delta p
$$

In an argument similar to that of Adler and Santiago discussed in Section 3.1.2, Maggiore then suggests that the two uncertainties, the usual one inversely proportional to the photon's energy 
and the additional one (52), should be linearly added to

$$
\Delta R_{H} \gtrsim \frac{1}{\Delta p}+\alpha G \Delta p,
$$

where the constant $\alpha$ would have to be fixed by using a specific theory. Minimizing the possible position uncertainty, one thus finds again a minimum error of $\approx \alpha l_{\mathrm{Pl}}$.

It is clear that the uncertainty Maggiore considered is of a different kind than the one considered by Mead, though both have the same origin. Maggiore's uncertainty is due to the impossibility of directly measuring a black hole without it emitting a particle that carries energy and thereby changing the black-hole-horizon area. The smaller the wavelength of the emitted particle, the larger the so-caused distortion. Mead's uncertainty is due to the formation of black holes if one uses probes of too high an energy, which limits the possible precision. But both uncertainties go back to the relation between a black hole's area and its mass.

\subsubsection{A device-independent limit for non-relativistic particles}

Even though the Heisenberg microscope is a very general instrument and the above considerations carry over to many other experiments, one may wonder if there is not some possibility to overcome the limitation of the Planck length by use of massive test particles that have smaller Compton wavelengths, or interferometers that allow one to improve on the limitations on measurement precisions set by the test particles' wavelengths. To fill in this gap, Calmet, Graesser and Hsu [72, 73] put forward an elegant device-independent argument. They first consider a discrete spacetime with a sub-Planckian spacing and then show that no experiment is able to rule out this possibility. The point of the argument is not the particular spacetime discreteness they consider, but that it cannot be ruled out in principle.

The setting is a position operator $\hat{x}$ with discrete eigenvalues $\left\{x_{i}\right\}$ that have a separation of order $l_{\mathrm{Pl}}$ or smaller. To exclude the model, one would have to measure position eigenvalues $x$ and $x^{\prime}$, for example, of some test particle of mass $M$, with $\left|x-x^{\prime}\right| \leq l_{\mathrm{Pl}}$. Assuming the non-relativistic Schrödinger equation without potential, the time-evolution of the position operator is given by $\mathrm{d} \hat{x}(t) / \mathrm{d} t=i[\hat{H}, \hat{x}(t)]=\hat{p} / M$, and thus

$$
\hat{x}(t)=\hat{x}(0)+\hat{p}(0) \frac{t}{M} .
$$

We want to measure the expectation value of position at two subsequent times in order to attempt to measure a spacing smaller than the Planck length. The spectra of any two Hermitian operators have to fulfill the inequality

$$
\Delta A \Delta B \geq \frac{1}{2 i}\langle[\hat{A}, \hat{B}]\rangle,
$$

where $\Delta$ denotes, as usual, the variance and $\langle\cdot\rangle$ the expectation value of the operator. From (54) one has

$$
[\hat{x}(0), \hat{x}(t)]=i \frac{t}{M},
$$

and thus

$$
\Delta x(0) \Delta x(t) \geq \frac{t}{2 M} .
$$

Since one needs to measure two positions to determine a distance, the minimal uncertainty to the distance measurement is

$$
\Delta x \geq \sqrt{\frac{t}{2 M}} .
$$


This is the same bound as previously discussed in Section 3.1.3 for the measurement of distances by help of a clock, yet we arrived here at this bound without making assumptions about exactly what is measured and how. If we take into account gravity, the argument can be completed similar to Wigner's and still without making assumptions about the type of measurement, as follows.

We use an apparatus of size $R$. To get the spacing as precise as possible, we would use a test particle of high mass. But then we will run into the, by now familiar, problem of black-hole formation when the mass becomes too large, so we have to require

$$
M<2 \frac{R}{G} .
$$

Thus, we cannot make the detector arbitrarily small. However, we also cannot make it arbitrarily large, since the components of the detector have to at least be in causal contact with the position we want to measure, and so $t>R$. Taken together, one finds

$$
\Delta x \geq \sqrt{\frac{t}{2 M}} \geq \sqrt{\frac{R}{2 M}} \geq \sqrt{G}
$$

and thus once again the possible precision of a position measurement is limited by the Planck length.

A similar argument was made by $\mathrm{Ng}$ and van Dam [238], who also pointed out that with this thought experiment one can obtain a scaling for the uncertainty with the third root of the size of the detector. If one adds the position uncertainty (58) from the non-vanishing commutator to the gravitational one, one finds

$$
\Delta x \gtrsim \sqrt{\frac{R}{2 M}}+G M
$$

Optimizing this expression with respect to the mass that yields a minimal uncertainty, one finds $M \sim\left(R / l_{\mathrm{Pl}}^{4}\right)^{1 / 3}$ (up to factors of order one) and, inserting this value of $M$ in (61), thus

$$
\Delta x \gtrsim\left(R l_{\mathrm{Pl}}^{2}\right)^{\frac{1}{3}} .
$$

Since $R$ too should be larger than the Planck scale this is, of course, consistent with the previouslyfound minimal uncertainty.

$\mathrm{Ng}$ and van Dam further argue that this uncertainty induces a minimum error in measurements of energy and momenta. By noting that the uncertainty $\Delta x$ of a length $R$ is indistinguishable from an uncertainty of the metric components used to measure the length, $\Delta x^{2}=R^{2} \Delta g$, the inequality (62) leads to

$$
\Delta g_{\mu \nu} \gtrsim\left(\frac{l_{\mathrm{Pl}}}{R}\right)^{\frac{2}{3}}
$$

But then again the metric couples to the stress-energy tensor $T_{\mu \nu}$, so this uncertainty for the metric further induces an uncertainty for the entries of $T_{\mu \nu}$

$$
\left(g_{\mu \nu}+\Delta g_{\mu \nu}\right) T^{\mu \nu}=g_{\mu \nu}\left(T^{\mu \nu}+\Delta T^{\mu \nu}\right) .
$$

Consider now using a test particle of momentum $p$ to probe the physics at scale $R$, thus $p \sim 1 / R$. Then its uncertainty would be on the order of

$$
\Delta p \gtrsim p\left(\frac{l_{\mathrm{Pl}}}{R}\right)^{\frac{2}{3}}=p\left(\frac{p}{m_{\mathrm{Pl}}}\right)^{\frac{2}{3}} .
$$


However, note that the scaling found by $\mathrm{Ng}$ and van Dam only follows if one works with the masses that minimize the uncertainty (61). Then, even if one uses a detector of the approximate extension of a cm, the corresponding mass of the 'particle' we have to work with would be about a ton. With such a mass one has to worry about very different uncertainties. For particles with masses below the Planck mass on the other hand, the size of the detector would have to be below the Planck length, which makes no sense since its extension too has to be subject to the minimal position uncertainty.

\subsubsection{Limits on the measurement of spacetime volumes}

The observant reader will have noticed that almost all of the above estimates have explicitly or implicitly made use of spherical symmetry. The one exception is the argument by Adler and Santiago in Section 3.1.2 that employed cylindrical symmetry. However, it was also assumed there that the length and the radius of the cylinder are of comparable size.

In the general case, when the dimensions of the test particle in different directions are very unequal, the Hoop conjecture does not forbid any one direction to be smaller than the Schwarzschild radius to prevent collapse of some matter distribution, as long as at least one other direction is larger than the Schwarzschild radius. The question then arises what limits that rely on black-hole formation can still be derived in the general case.

A heuristic motivation of the following argument can be found in [101], but here we will follow the more detailed argument by Tomassini and Viaggiu [307]. In the absence of spherical symmetry, one may still use Penrose's isoperimetric-type conjecture, according to which the apparent horizon is always smaller than or equal to the event horizon, which in turn is smaller than or equal to $16 \pi G^{2} \omega^{2}$, where $\omega$ is as before the energy of the test particle.

Then, without spherical symmetry the requirement that no black hole ruins our ability to resolve short distances is weakened from the energy distribution having a radius larger than the Schwarzschild radius, to the requirement that the area $A$, which encloses $\omega$ is large enough to prevent Penrose's condition for horizon formation

$$
A \geq 16 \pi G^{2} \omega^{2} .
$$

The test particle interacts during a time $\Delta T$ that, by the normal uncertainty principle, is larger than $1 /(2 \omega)$. Taking into account this uncertainty on the energy, one has

$$
A(\Delta T)^{2} \geq 4 \pi G^{2} .
$$

Now we have to make some assumption for the geometry of the object, which will inevitably be a crude estimate. While an exact bound will depend on the shape of the matter distribution, we will here just be interested in obtaining a bound that depends on the three different spatial extensions, and is qualitatively correct. To that end, we assume the mass distribution fits into some smallest box with side-lengths $\Delta x^{1}, \Delta x^{2}, \Delta x^{3}$, which is similar to the limiting area

$$
A \sim \frac{\Delta x^{1} \Delta x^{2}+\Delta x^{1} \Delta x^{3}+\Delta x^{2} \Delta x^{3}}{\alpha^{2}},
$$

where we added some constant $\alpha$ to take into account different possible geometries. A comparison with the spherical case, $\Delta x^{i}=2 R$, fixes $\alpha^{2}=3 / \pi$. With Eq. (67) one obtains

$$
(\Delta t)^{2}\left(\Delta x^{1} \Delta x^{2}+\Delta x^{1} \Delta x^{3}+\Delta x^{2} \Delta x^{3}\right) \geq 12 l_{\mathrm{p}}^{4} .
$$

Since

$$
\left(\Delta x^{1}+\Delta x^{2}+\Delta x^{3}\right)^{2} \geq \Delta x^{1} \Delta x^{2}+\Delta x^{1} \Delta x^{3}+\Delta x^{2} \Delta x^{3}
$$


one also has

$$
\Delta t\left(\Delta x^{1}+\Delta x^{2}+\Delta x^{3}\right) \geq 12 l_{\mathrm{p}}^{2},
$$

which confirms the limit obtained earlier by heuristic reasoning in [101].

Thus, as anticipated, taking into account that a black hole must not necessarily form if the spatial extension of a matter distribution is smaller than the Schwarzschild radius in only one direction, the uncertainty we arrive at here depends on the extension in all three directions, rather than applying separately to each of them. Here we have replaced $\omega$ by the inverse of $\Delta T$, rather than combining with Eq. (2), but this is just a matter of presentation.

Since the bound on the volumes (71) follows from the bounds on spatial and temporal intervals we found above, the relevant question here is not whether ?? is fulfilled, but whether the bound $\Delta x \gtrsim l_{\mathrm{Pl}}$ can be violated [165].

To address that question, note that the quantities $\Delta x_{i}$ in the above argument by Tomassini and Viaggiu differ from the ones we derived bounds for in Sections 3.1.1-3.1.6. Previously, the $\Delta x$ was the precision by which one can measure the position of a particle with help of the test particle. Here, the $\Delta x_{i}$ are the smallest possible extensions of the test particle (in the rest frame), which with spherical symmetry would just be the Schwarzschild radius. The step in which one studies the motion of the measured particle that is induced by the gravitational field of the test particle is missing in this argument. Thus, while the above estimate correctly points out the relevance of non-spherical symmetries, the argument does not support the conclusion that it is possible to test spatial distances to arbitrary precision.

The main obstacle to completion of this argument is that in the context of quantum field theory we are eventually dealing with particles probing particles. To avoid spherical symmetry, we would need different objects as probes, which would require more information about the fundamental nature of matter. We will come back to this point in Section 3.2.3.

\subsection{String theory}

String theory is one of the leading candidates for a theory of quantum gravity. Many textbooks have been dedicated to the topic, and the interested reader can also find excellent resources online [187, 278, 235, 299]. For the following we will not need many details. Most importantly, we need to know that a string is described by a 2-dimensional surface swept out in a higher-dimensional spacetime. The total number of spatial dimensions that supersymmetric string theory requires for consistency is nine, i.e., there are six spatial dimensions in addition to the three we are used to. In the following we will denote the total number of dimensions, both time and space-like, with $D$. In this Subsection, Greek indices run from 0 to $D$.

The two-dimensional surface swept out by the string in the $D$-dimensional spacetime is referred to as the 'worldsheet,' will be denoted by $X^{\nu}$, and will be parameterized by (dimensionless) parameters $\sigma$ and $\tau$, where $\tau$ is its time-like direction, and $\sigma$ runs conventionally from 0 to $2 \pi$. A string has discrete excitations, and its state can be expanded in a series of these excitations plus the motion of the center of mass. Due to conformal invariance, the worldsheet carries a complex structure and thus becomes a Riemann surface, whose complex coordinates we will denote with $z$ and $\bar{z}$. Scattering amplitudes in string theory are a sum over such surfaces.

In the following $l_{\mathrm{s}}$ is the string scale, and $\alpha^{\prime}=l_{\mathrm{s}}^{2}$. The string scale is related to the Planck scale by $l_{\mathrm{Pl}}=g_{\mathrm{s}}^{1 / 4} l_{\mathrm{s}}$, where $g_{\mathrm{s}}$ is the string coupling constant. Contrary to what the name suggests, the string coupling constant is not constant, but depends on the value of a scalar field known as the dilaton.

To avoid conflict with observation, the additional spatial dimensions of string theory have to be compactified. The compactification scale is usually thought to be about the Planck length, and far below experimental accessibility. The possibility that the extensions of the extra dimensions (or at 
least some of them) might be much larger than the Planck length and thus possibly experimentally accessible, has been studied in models with a large compactification volume and lowered Planck scale, see, e.g., [1]. We will not discuss these models here, but mention in passing that they demonstrate the possibility that the 'true' higher-dimensional Planck mass is in fact much smaller than $m_{\mathrm{Pl}}$, and correspondingly the 'true' higher-dimensional Planck length, and with it the minimal length, much larger than $l_{\mathrm{Pl}}$. That such possibilities exist means, whether or not the model with extra dimensions are realized in nature, that we should, in principle, consider the minimal length a free parameter that has to be constrained by experiment.

String theory is also one of the motivations to look into non-commutative geometries. Noncommutative geometry will be discussed separately in Section 3.6. A section on matrix models will be included in a future update.

\subsubsection{Generalized uncertainty}

The following argument, put forward by Susskind [297, 298], will provide us with an insightful examination that illustrates how a string is different from a point particle and what consequences this difference has for our ability to resolve structures at shortest distances. We consider a free string in light cone coordinates, $X_{ \pm}=\left(X^{0} \pm X^{1}\right) / \sqrt{2}$ with the parameterization $X_{+}=2 l_{\mathrm{s}}^{2} P_{+} \tau$, where $P_{+}$is the momentum in the direction $X_{+}$and constant along the string. In the light-cone gauge, the string has no oscillations in the $X_{+}$direction by construction.

The transverse dimensions are the remaining $X^{i}$ with $i>1$. The normal mode decomposition of the transverse coordinates has the form

$$
X^{i}(\sigma, \tau)=x^{i}(\sigma, \tau)+\mathrm{i} \sqrt{\frac{\alpha^{\prime}}{2}} \sum_{n \neq 0}\left(\frac{\alpha_{n}^{i}}{n} e^{\mathrm{i} n(\tau+\sigma)}+\frac{\tilde{\alpha}_{n}^{i}}{n} e^{\mathrm{i} n(\tau-\sigma)}\right),
$$

where $x^{i}$ is the (transverse location of) the center of mass of the string. The coefficients $\alpha_{n}^{i}$ and $\tilde{\alpha}_{n}^{i}$ are normalized to $\left[\alpha_{n}^{i}, \alpha_{m}^{j}\right]=\left[\tilde{\alpha}_{n}^{i}, \tilde{\alpha}_{m}^{j}\right]=-\mathrm{i} m \delta^{i j} \delta_{m,-n}$, and $\left[\tilde{\alpha}_{n}^{i}, \alpha_{m}^{j}\right]=0$. Since the components $X^{\nu}$ are real, the coefficients have to fulfill the relations $\left(\alpha_{n}^{i}\right)^{*}=\alpha_{-n}^{i}$ and $\left(\tilde{\alpha}_{n}^{i}\right)^{*}=\tilde{\alpha}_{-n}^{i}$.

We can then estimate the transverse size $\Delta X_{\perp}$ of the string by

$$
\left(\Delta X_{\perp}\right)^{2}=\left\langle\sum_{i=2}^{D}\left(X^{i}-x^{i}\right)^{2}\right\rangle,
$$

which, in the ground state, yields an infinite sum

$$
\left(\Delta X_{\perp}\right)^{2} \sim l_{\mathrm{s}}^{2} \sum_{n} \frac{1}{n}
$$

This sum is logarithmically divergent because modes with arbitrarily high frequency are being summed over. To get rid of this unphysical divergence, we note that testing the string with some energy $E$, which corresponds to some resolution time $\Delta T=1 / E$, allows us to cut off modes with frequency $>1 / \Delta T$ or mode number $n \sim l_{\mathrm{s}} E$. Then, for large $n$, the sum becomes approximately

$$
\Delta X_{\perp}^{2} \approx l_{\mathrm{s}}^{2} \log \left(l_{\mathrm{s}} E\right) .
$$

Thus, the transverse extension of the string grows with the energy that the string is tested by, though only very slowly so.

To determine the spread in the longitudinal direction $X_{-}$, one needs to know that in light-cone coordinates the constraint equations on the string have the consequence that $X_{-}$is related to the 
transverse directions so that it is given in terms of the light-cone Virasoro generators

$$
X_{-}(\sigma, \tau)=x^{-}(\sigma, \tau)+\frac{\mathrm{i}}{P_{+}} \sum_{n \neq 0}\left(\frac{L_{n}}{n} e^{\mathrm{i} n(\tau+\sigma)}+\frac{\tilde{L}_{n}}{n} e^{\mathrm{i} n(\tau-\sigma)}\right)
$$

where now $L_{n}$ and $\tilde{L}_{n}$ fulfill the Virasoro algebra. Therefore, the longitudinal spread in the ground state gains a factor $\propto n^{2}$ over the transverse case, and diverges as

$$
\left(\Delta X_{-}\right)^{2} \sim \frac{1}{P_{+}^{2}} \sum_{n} n
$$

Again, this result has an unphysical divergence, that we deal with the same way as before by taking into account a finite resolution $\Delta T$, corresponding to the inverse of the energy by which the string is probed. Then one finds for large $n$ approximately

$$
\left(\Delta X_{-}\right)^{2} \approx\left(\frac{l_{\mathrm{s}}}{P_{+}}\right)^{2} E^{2} .
$$

Thus, this heuristic argument suggests that the longitudinal spread of the string grows linearly with the energy at which it is probed.

The above heuristic argument is supported by many rigorous calculations. That string scattering leads to a modification of the Heisenberg uncertainty relation has been shown in several studies of string scattering at high energies performed in the late 1980s [140, 310, 228]. Gross and Mende [140] put forward a now well-known analysis of the classic solution for the trajectories of a string worldsheet describing a scattering event with external momenta $p_{i}^{\nu}$. In the lowest tree approximation they found for the extension of the string

$$
x^{\nu}(z, \bar{z}) \approx l_{\mathrm{s}}^{2} \sum_{i} p_{i}^{\nu} \log \left|z-z_{i}\right|
$$

plus terms that are suppressed in energy relative to the first. Here, $z_{i}$ are the positions of the vertex operators on the Riemann surface corresponding to the asymptotic states with momenta $p_{i}^{\nu}$. Thus, as previously, the extension grows linearly with the energy. One also finds that the surface of the string grows with $E / N$, where $N$ is the genus of the expansion, and that the fixed angle scattering amplitude at high energies falls exponentially with the square of the center-of-mass energy $s$ (times $\left.l_{\mathrm{s}}^{2}\right)$.

One can interpret this spread of the string in terms of a GUP by taking into account that at high energies the spread grows linearly with the energy. Together with the normal uncertainty, one obtains

$$
\Delta x^{\nu} \Delta p^{\nu} \gtrsim 1+l_{\mathrm{s}} E
$$

again the GUP that gives rise to a minimally-possible spatial resolution.

However, the exponential fall-off of the tree amplitude depends on the genus of the expansion, and is dominated by the large $N$ contributions because these decrease slower. The Borel resummation of the series has been calculated in [228] and it was found that the tree level approximation is valid only for an intermediate range of energies, and for $s l_{\mathrm{s}}^{2} \gg g_{\mathrm{s}}^{-4 / 3}$ the amplitude decreases much slower than the tree-level result would lead one to expect. Yoneya [318] has furthermore argued that this behavior does not properly take into account non-perturbative effects, and thus the generalized uncertainty should not be regarded as generally valid in string theory. We will discuss this in Section 3.2.3. 
It has been proposed that the resistance of the string to attempts to localize it plays a role in resolving the black-hole information-loss paradox [204]. In fact, one can wonder if the high energy behavior of the string acts against and eventually prevents the formation of black holes in elementary particle collisions. It has been suggested in $[10,9,11]$ that string effects might become important at impact parameters far greater than those required to form black holes, opening up the possibility that black holes might not form.

The completely opposite point of view, that high energy scattering is ultimately entirely dominated by black-hole production, has also been put forward [48, 131]. Giddings and Thomas found an indication of how gravity prevents probes of distance shorter than the Planck scale [131] and discussed the 'the end of short-distance physics'; Banks aptly named it 'asymptotic darkness' [47]. A recent study of string scattering at high energies [127] found no evidence that the extendedness of the string interferes with black-hole formation. The subject of string scattering in the trans-Planckian regime is subject of ongoing research, see, e.g., [12, 90, 130] and references therein.

Let us also briefly mention that the spread of the string just discussed should not be confused with the length of the string. (For a schematic illustration see Figure 2.) The length of a string in the transverse direction is

$$
L=\int \mathrm{d} \sigma\left(\partial_{\sigma} X^{i} \partial_{\sigma} X^{i}\right)^{2},
$$

where the sum is taken in the transverse direction, and has been studied numerically in [173]. In this study, it has been shown that when one increases the cut-off on the modes, the string becomes space-filling, and fills space densely (i.e., it comes arbitrarily close to any point in space).

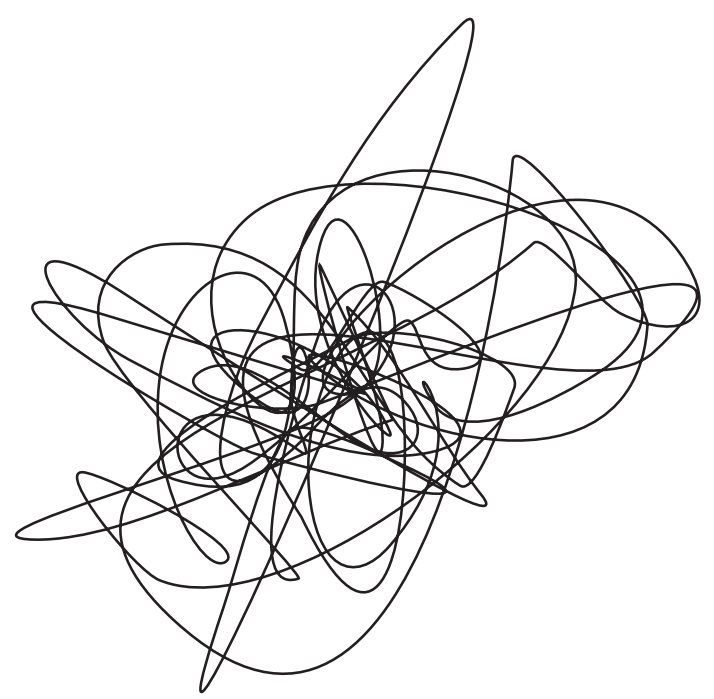

Figure 2: The length of a string is not the same as its average extension. The lengths of strings in the groundstate were studied in [173].

\subsubsection{Spacetime uncertainty}

Yoneya [318] argued that the GUP in string theory is not generally valid. To begin with, it is not clear whether the Borel resummation of the perturbative expansion leads to correct nonperturbative results. And, after the original works on the generalized uncertainty in string theory, it has become understood that string theory gives rise to higher-dimensional membranes that are 
dynamical objects in their own right. These higher-dimensional membranes significantly change the picture painted by high energy string scattering, as we will see in 3.2.3. However, even if the GUP is not generally valid, there might be a different uncertainty principle that string theory conforms to, that is a spacetime uncertainty of the form

$$
\Delta X \Delta T \gtrsim l_{\mathrm{s}}^{2}
$$

This spacetime uncertainty has been motivated by Yoneya to arise from conformal symmetry $[317,318]$ as follows.

Suppose we are dealing with a Riemann surface with metric $\mathrm{d} s=\rho(z, \bar{z})|\mathrm{d} z|$ that parameterizes the string. In string theory, these surfaces appear in all path integrals and thus amplitudes, and they are thus of central importance for all possible processes. Let us denote with $\Omega$ a finite region in that surface, and with $\Gamma$ the set of all curves in $\Omega$. The length of some curve $\gamma \in \Gamma$ is then $L(\gamma, \rho)=\int_{\gamma} \rho|\mathrm{d} z|$. However, this length that we are used to from differential geometry is not conformally invariant. To find a length that captures only the physically-relevant information, one can use a distance measure known as the 'extremal length' $\lambda_{\Omega}$

$$
\lambda_{\Omega}(\Lambda)=\sup _{\rho} \frac{L(\Omega, \rho)^{2}}{A(\Omega, \rho)},
$$

with

$$
L(\Gamma, \rho)=\inf _{\gamma \in \Lambda} L(\gamma, \rho), \quad A(\Omega, \rho)=\int_{\Omega} \rho^{2} \mathrm{~d} z \mathrm{~d} \bar{z} .
$$

The so-constructed length is dimensionless and conformally invariant. For simplicity, we assume that $\Omega$ is a generic polygon with four sides and four corners, with pairs of opposite sides named $\alpha, \alpha^{\prime}$ and $\beta, \beta^{\prime}$. Any more complicated shape can be assembled from such polygons. Let $\Gamma$ be the set of all curves connecting $\alpha$ with $\alpha^{\prime}$ and $\Gamma^{*}$ the set of all curves connecting $\beta$ with $\beta^{\prime}$. The extremal lengths $\lambda_{\Omega}(\Gamma)$ and $\lambda_{\Omega}\left(\Gamma^{*}\right)$ then fulfill property $[317,318]$

$$
\lambda_{\Omega}\left(\Gamma^{*}\right) \lambda_{\Omega}(\Gamma)=1
$$

Conformal invariance allows us to deform the polygon, so instead of a general four-sided polygon, we can consider a rectangle in particular, where the Euclidean length of the sides $\left(\alpha, \alpha^{\prime}\right)$ will be named $a$ and that of sides $\left(\beta, \beta^{\prime}\right)$ will be named $b$. With a Minkowski metric, one of these directions would be timelike and one spacelike. Then the extremal lengths are [317, 318]

$$
\lambda_{\Omega}\left(\Gamma^{*}\right)=\frac{b}{a}, \quad \lambda_{\Omega}(\Gamma)=\frac{a}{b} .
$$

Armed with this length measure, let us consider the Euclidean path integral in the conformal gauge $\left(g_{\mu \nu}=\eta_{\mu \nu}\right)$ with the action

$$
\frac{1}{l_{\mathrm{S}}^{2}} \int_{\Omega} \mathrm{d} z \mathrm{~d} \bar{z} \partial_{z} X^{\nu} \partial_{\bar{z}} X^{\nu}
$$

(Equal indices are summed over). As before, $X$ are the target space coordinates of the string worldsheet. We now decompose the coordinate $z$ into its real and imaginary part $\sigma_{1}=\operatorname{Re}(z), \sigma_{2}=$ $\operatorname{Im}(z)$, and consider a rectangular piece of the surface with the boundary conditions

$$
\begin{aligned}
& X^{\nu}\left(0, \sigma_{2}\right)=X^{\nu}\left(a, \sigma_{2}\right)=\delta^{\nu 2} B \frac{\sigma_{2}}{b}, \\
& X^{\nu}\left(\sigma_{1}, 0\right)=X^{\nu}\left(\sigma_{1}, b\right)=\delta^{\nu 1} A \frac{\sigma_{1}}{a} .
\end{aligned}
$$


If one integrates over the rectangular region, the action contains a factor $a b\left((B / b)^{2}+(A / a)^{2}\right)$ and the path integral thus contains a factor of the form

$$
\exp \left(-\frac{1}{l_{\mathrm{s}}^{2}}\left(\frac{A^{2}}{\lambda(\Gamma)}+\frac{B^{2}}{\lambda\left(\Gamma^{*}\right)}\right)\right) .
$$

Thus, the width of these contributions is given by the extremal length times the string scale, which quantifies the variance of $A$ and $B$ by

$$
\Delta A \sim l_{\mathrm{s}} \sqrt{\lambda(\Gamma)}, \quad \Delta B \sim l_{\mathrm{s}} \sqrt{\lambda\left(\Gamma^{*}\right)} .
$$

In particular the product of both satisfies the condition

$$
\Delta A \Delta B \sim l_{\mathrm{s}}^{2} .
$$

Thus, probing short distances along the spatial and temporal directions simultaneously is not possible to arbitrary precision, lending support to the existence of a spacetime uncertainty of the form (82). Yoneya notes [318] that this argument cannot in this simple fashion be carried over to more complicated shapes. Thus, at present the spacetime uncertainty has the status of a conjecture. However, the power of this argument rests in it only relying on conformal invariance, which makes it plausible that, in contrast to the GUP, it is universally and non-perturbatively valid.

\subsubsection{Taking into account Dp-Branes}

The endpoints of open strings obey boundary conditions, either of the Neumann type or of the Dirichlet type or a mixture of both. For Dirichlet boundary conditions, the submanifold on which open strings end is called a Dirichlet brane, or Dp-brane for short, where $\mathrm{p}$ is an integer denoting the dimension of the submanifold. A D0-brane is a point, sometimes called a D-particle; a D1-brane is a one-dimensional object, also called a D-string; and so on, all the way up to D9-branes.

These higher-dimensional objects that arise in string theory have a dynamics in their own right, and have given rise to a great many insights, especially with respect to dualities between different sectors of the theory, and the study of higher-dimensional black holes [170, 45].

Dp-branes have a tension of $T_{p}=1 /\left(g_{\mathrm{s}} \mathrm{s}_{\mathrm{s}}^{p+1}\right)$; that is, in the weak coupling limit, they become very rigid. Thus, one might suspect $\mathrm{D}$-particles to show evidence for structure on distances at least down to $l_{\mathrm{s}} g_{\mathrm{s}}$.

Taking into account the scattering of Dp-branes indeed changes the conclusions we could draw from the earlier-discussed thought experiments. We have seen that this was already the case for strings, but we can expect that Dp-branes change the picture even more dramatically. At high energies, strings can convert energy into potential energy, thereby increasing their extension and counteracting the attempt to probe small distances. Therefore, strings do not make good candidates to probe small structures, and to probe the structures of Dp-branes, one would best scatter them off each other. As Bachas put it [45], the "small dynamical scale of D-particles cannot be seen by using fundamental-string probes - one cannot probe a needle with a jelly pudding, only with a second needle!"

That with Dp-branes new scaling behaviors enter the physics of shortest distances has been pointed out by Shenker [283], and in particular the D-particle scattering has been studied in great detail by Douglas et al. [103]. It was shown there that indeed slow moving D-particles can probe distances below the (ten-dimensional) Planck scale and even below the string scale. For these D-particles, it has been found that structures exist down to $g_{\mathrm{s}}^{1 / 3} l_{\mathrm{s}}$. 
To get a feeling for the scales involved here, let us first reconsider the scaling arguments on blackhole formation, now in a higher-dimensional spacetime. The Newtonian potential $\phi$ of a higherdimensional point charge with energy $E$, or the perturbation of $g_{00}=1+2 \phi$, in $D$ dimensions, is qualitatively of the form

$$
\phi \sim \frac{G_{D} E}{(\Delta x)^{D-3}}
$$

where $\Delta x$ is the spatial extension, and $G_{D}$ is the $D$-dimensional Newton's constant, related to the Planck length as $G_{D}=l_{\mathrm{Pl}}^{D-2}$. Thus, the horizon or the zero of $g_{00}$ is located at

$$
\Delta x \sim\left(G_{D} E\right)^{\frac{1}{D-3}}
$$

With $E \sim 1 / \Delta t$, for some time by which we test the geometry, to prevent black-hole formation for $D=10$, one thus has to require

$$
(\Delta t)(\Delta x)^{7} \gtrsim G_{10}=g_{\mathrm{s}}^{2} l_{\mathrm{s}}^{8},
$$

re-expressed in terms of string coupling and tension. We see that in the weak coupling limit, this lower bound can be small, in particular it can be much below the string scale.

This relation between spatial and temporal resolution can now be contrasted with the spacetime uncertainty (82), that sets the limits below which the classical notion of spacetime ceases to make sense. Both of these limits are shown in Figure 3 for comparison. The curves meet at

$$
\Delta x_{\min } \sim l_{\mathrm{s}} g_{\mathrm{s}}^{1 / 3}, \quad \Delta t_{\min } \sim l_{\mathrm{s}} g_{\mathrm{s}}^{-1 / 3}
$$

If we were to push our limits along the bound set by the spacetime uncertainty (red, solid line), then the best possible spatial resolution we could reach lies at $\Delta x_{\min }$, beyond which black-hole production takes over. Below the spacetime uncertainty limit, it would actually become meaningless to talk about black holes that resemble any classical object.

At first sight, this argument seems to suffer from the same problem as the previously examined argument for volumes in Section 3.1.7. Rather than combining $\Delta t$ with $\Delta x$ to arrive at a weaker bound than each alone would have to obey, one would have to show that in fact $\Delta x$ can become arbitrarily small. And, since the argument from black-hole collapse in 10 dimensions is essentially the same as Mead's in 4 dimensions, just with a different $r$-dependence of $\phi$, if one would consider point particles in 10 dimensions, one finds along the same line of reasoning as in Section 3.1.2, that actually $\Delta t \gtrsim l_{\mathrm{Pl}}$ and $\Delta x \gtrsim l_{\mathrm{Pl}}$.

However, here the situation is very different because fundamentally the objects we are dealing with are not particles but strings, and the interaction between Dp-branes is mediated by strings stretched between them. It is an inherently different behavior than what we can expect from the classical gravitational attraction between point particles. At low string coupling, the coupling of gravity is weak and in this limit then, the backreaction of the branes on the background becomes negligible. For these reasons, the D-particles distort each other less than point particles in a quantum field theory would, and this is what allows one to use them to probe very short distances.

The following estimate from [318] sheds light on the scales that we can test with D-particles in particular. Suppose we use D-particles with velocity $v$ and mass $m_{0}=1 /\left(l_{\mathrm{s}} g_{\mathrm{s}}\right)$ to probe a distance of size $\Delta x$ in time $\Delta t$. Since $v \Delta t \sim \Delta x$, the uncertainty (94) gives

$$
(\Delta x)^{8} \gtrsim v g_{\mathrm{s}}^{2} l_{\mathrm{s}}^{8}
$$

thus, to probe very short distances one has to use slow D-particles. 


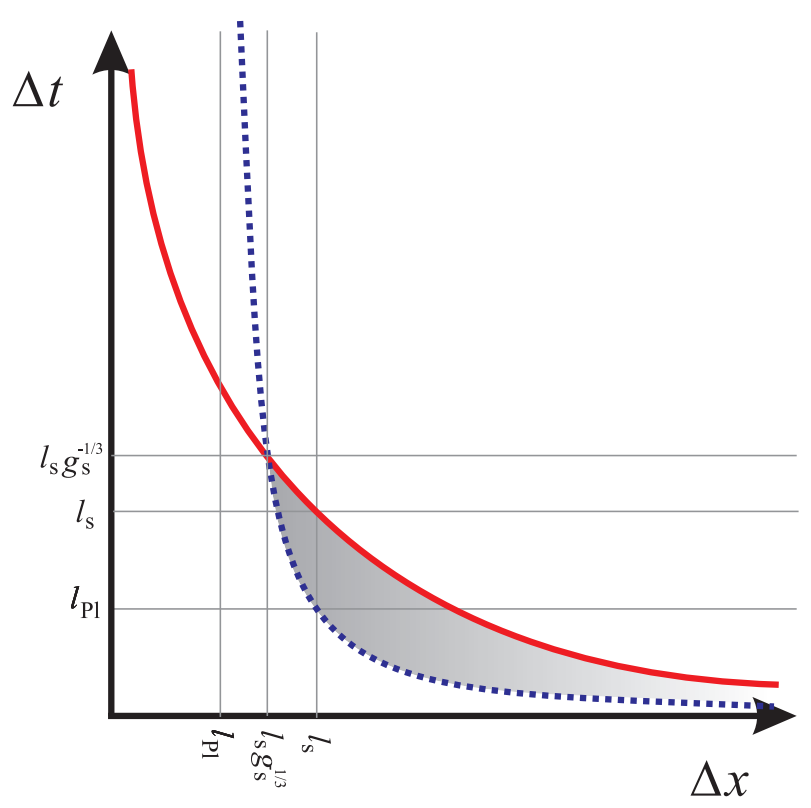

Figure 3: Spacetime uncertainty (red, solid) vs uncertainty from spherical black holes (blue, dotted) in $D=10$ dimensions, for $g_{\mathrm{s}}<1$ (left) and $g_{\mathrm{s}}>1$ (right). After [318], Figure 1. Below the bound from spacetime uncertainty yet above the black-hole bound that hides short-distance physics (shaded region), the concept of classical geometry becomes meaningless.

But if the D-particle is slow, then its wavefunction behaves like that of a massive non-relativistic particle, so we have to take into account that the width spreads with time. For this, we can use the earlier-discussed bound Eq. (58)

$$
\Delta x_{\text {spread }} \gtrsim \sqrt{\frac{\Delta t}{2 m_{0}}},
$$

or

$$
\Delta x_{\text {spread }} \gtrsim \frac{l_{\mathrm{s}} g_{\mathrm{s}}}{2 v} .
$$

If we add the uncertainties (96) and (98) and minimize the sum with respect to $v$, we find that the spatial uncertainty is minimal for

$$
v \sim g_{\mathrm{s}}^{2 / 3} .
$$

Thus, the total spatial uncertainty is bounded by

$$
\Delta x \gtrsim l_{\mathrm{s}} g_{\mathrm{s}}^{1 / 3},
$$

and with this one also has

$$
\Delta t \gtrsim l_{\mathrm{s}} g_{\mathrm{s}}^{-1 / 3},
$$

which are the scales that we already identified in (95) to be those of the best possible resolution compatible with the spacetime uncertainty. Thus, we see that the D-particles saturate the spacetime uncertainty bound and they can be used to test these short distances. 
Table 1: Analogy between scales involved in D-particle scattering and the hydrogen atom. After [103].

\begin{tabular}{ll}
\hline Electron & $\boldsymbol{D}$-particle \\
\hline mass $m_{\mathrm{e}}$ & mass $m_{0}=1 /\left(l_{\mathrm{s}} g_{\mathrm{s}}\right)$ \\
Compton wavelength $1 / m_{\mathrm{e}}$ & Compton wavelength $1 / m_{0} \sim g_{\mathrm{s}} l_{\mathrm{s}}$ \\
velocity $\alpha$ & velocity $v=g_{\mathrm{s}}^{2 / 3}$ \\
Bohr radius $\sim 1 /\left(\alpha m_{\mathrm{e}}\right)$ & size of resonance $1 /\left(m_{0} v\right) \sim g_{\mathrm{s}}^{1 / 3} l_{\mathrm{s}}$ \\
energy levels $\sim \alpha^{2} m_{\mathrm{e}}$ & resonance energy $\sim m_{0} v^{2} \sim g_{\mathrm{s}}^{1 / 3} / l_{\mathrm{s}}$ \\
fine structure $\sim \alpha^{4} m_{\mathrm{e}}$ & energy shifts $\sim m_{0} v^{4} \sim g_{\mathrm{s}}^{5 / 3} / l_{\mathrm{s}}$ \\
\hline
\end{tabular}

D-particle scattering has been studied in [103] by use of a quantum mechanical toy model in which the two particles are interacting by (unexcited) open strings stretched between them. The open strings create a linear potential between the branes. At moderate velocities, repeated collisions can take place, since the probability for all the open strings to annihilate between one collision and the next is small. At $v \sim g_{\mathrm{s}}^{2 / 3}$, the time between collisions is on the order of $\Delta t \sim l_{\mathrm{s}} g^{-1 / 3}$, corresponding to a resonance of width $\Gamma \sim g_{\mathrm{s}}^{1 / 3} / l_{\mathrm{s}}$. By considering the conversion of kinetic energy into the potential of the strings, one sees that the particles reach a maximal separation of $\Delta x \sim l_{\mathrm{s}} g_{\mathrm{s}}^{-1 / 3}$, realizing a test of the scales found above.

Douglas et al. [103] offered a useful analogy of the involved scales to atomic physics; see Table (1). The electron in a hydrogen atom moves with velocity determined by the fine-structure constant $\alpha$, from which it follows the characteristic size of the atom. For the D-particles, this corresponds to the maximal separation in the repeated collisions. The analogy may be carried further than that in that higher-order corrections should lead to energy shifts.

The possibility to resolve such short distances with D-branes have been studied in many more calculations; for a summary, see, for example, [45] and references therein. For our purposes, this estimate of scales will be sufficient. We take away that D-branes, should they exist, would allow us to probe distances down to $\Delta x \sim g_{\mathrm{s}}^{1 / 3} l_{\mathrm{s}}$.

\subsubsection{T-duality}

In the presence of compactified spacelike dimensions, a string can acquire an entirely new property: It can wrap around the compactified dimension. The number of times it wraps around, labeled by the integer $w$, is called the 'winding-number.' For simplicity, let us consider only one additional dimension, compactified on a radius $R$. Then, in the direction of this coordinate, the string has to obey the boundary condition

$$
X^{4}(\tau, \sigma+2 \pi)=X^{4}(\tau, \sigma)+2 \pi w R .
$$

The momentum in the direction of the additional coordinate is quantized in multiples of $1 / R$, so the expansion (compare to Eq. (72)) reads

$$
X^{4}(\tau, \sigma)=x_{0}^{4}+\frac{n l_{\mathrm{s}}^{2}}{R} \tau+w R \sigma+\mathrm{i} \frac{\alpha^{\prime}}{2} \sum_{n \neq 0}\left(\frac{\alpha_{n}^{i}}{n} e^{\mathrm{i} n(\tau+\sigma)}+\frac{\tilde{\alpha}_{n}^{i}}{n} e^{\mathrm{i} n(\tau-\sigma)}\right),
$$

where $x_{0}^{4}$ is some initial value. The momentum $P^{4}=\partial_{\tau} x^{4} /\left(l_{\mathrm{s}}^{2}\right)$ is then

$$
P^{4}(\tau, \sigma)=\frac{n}{R} \tau+\frac{\mathrm{i}}{\sqrt{2} l_{\mathrm{s}}} \sum_{n \neq 0}\left(\alpha_{n}^{i} e^{\mathrm{i} n(\tau+\sigma)}+\tilde{\alpha}_{n}^{i} e^{\mathrm{i} n(\tau-\sigma)}\right) .
$$


The total energy of the quantized string with excitation $n$ and winding number $w$ is formally divergent, due to the contribution of all the oscillator's zero point energies, and has to be renormalized. After renormalization, the energy is

$$
\begin{aligned}
& E^{2}=\sum_{\mu=0}^{3} P^{\mu} P_{\mu}+m^{2} \quad \text { with } \\
& m^{2}=\frac{n^{2}}{R^{2}}+w^{2} \frac{R^{2}}{\alpha^{\prime 2}}+\frac{2}{\alpha^{\prime}}\left(N+\tilde{N}-\frac{D-2}{12}\right),
\end{aligned}
$$

where $\mu$ runs over the non-compactified coordinates, and $N$ and $\tilde{N}$ are the levels of excitations of the left and right moving modes. Level matching requires $n w+N-\tilde{N}=0$. In addition to the normal contribution from the linear momentum, the string energy thus has a geometricallyquantized contribution from the momentum into the extra dimension(s), labeled with $n$, an energy from the winding (more winding stretches the string and thus needs energy), labeled with $w$, and a renormalized contribution from the Casimir energy. The important thing to note here is that this expression is invariant under the exchange

$$
R \leftrightarrow \frac{l_{\mathrm{s}}^{2}}{R}, \quad n \leftrightarrow w,
$$

i.e., an exchange of winding modes with excitations leaves mass spectrum invariant.

This symmetry is known as target-space duality, or T-duality for short. It carries over to multiples extra dimensions, and can be shown to hold not only for the free string but also during interactions. This means that for the string a distance below the string scale $\sim l_{\mathrm{s}}$ is meaningless because it corresponds to a distance larger than that; pictorially, a string that is highly excited also has enough energy to stretch and wrap around the extra dimension. We have seen in Section 3.2.3 that Dp-branes overcome limitations of string scattering, but T-duality is a simple yet powerful way to understand why the ability of strings to resolves short distances is limited.

This characteristic property of string theory has motivated a model that incorporates T-duality and compact extra dimensions into an effective path integral approach for a particle-like object that is described by the center-of-mass of the string, yet with a modified Green's function, suggested in [285, 111, 291].

In this approach it is assumed that the elementary constituents of matter are fundamentally strings that propagate in a higher dimensional spacetime with compactified additional dimensions, so that the strings can have excitations and winding numbers. By taking into account the excitations and winding numbers, Fontanini et al. [285, 111, 291] derive a modified Green's function for a scalar field. In the resulting double sum over $n$ and $w$, the contribution from the $n=0$ and $w=0$ zero modes is dropped. Note that this discards all massless modes as one sees from Eq. (106). As a result, the Green's function obtained in this way no longer has the usual contribution

$$
G(x, y)=-\frac{1}{(x-y)^{2}}
$$

Instead, one finds in momentum space

$$
G(p)=\sum_{N=0}^{\infty} \sum_{w, n=1}^{\infty} \frac{n l_{0}}{\sqrt{p^{2}+m^{2}}} K_{1}\left(n l_{0} \sqrt{p^{2}+m^{2}}\right),
$$

where the mass term is given by Eq. (106) and a function of $N, n$ and $w$. Here, $K_{1}$ is the modified Bessel function of the first kind, and $l_{0}=2 \pi R$ is the compactification scale of the extra dimensions. 
For $n=w=1$ and $N=0$, in the limit where $p^{2} \gg m^{2}$ and the argument of $K_{1}$ is large compared to $1, p^{2} \gg 1 / l_{0}^{2}$, the modified Bessel function can be approximated by

$$
K_{1} \rightarrow \frac{4 \pi^{2}}{\sqrt{p l_{0}}} \exp \left(-l_{0} \sqrt{p^{2}}\right)
$$

and, in that limit, the term in the sum (109) of the Green's function takes the form

$$
\rightarrow \frac{\sqrt{l_{0}}}{p^{3 / 2}} \exp \left(-l_{0} \sqrt{p^{2}}\right)
$$

Thus, each term of the modified Green's function falls off exponentially if the energies are large enough. The Fourier transform of this limit of the momentum space propagator is

$$
G(x, y) \approx \frac{1}{4 \pi^{2}} \frac{1}{(x-y)^{2}+l_{0}^{2}},
$$

and one thus finds that the spacetime distance in the propagator acquires a finite correction term, which one can interpret as a 'zero point length', at least in the Euclidean case.

It has been argued in [285] that this "captures the leading order correction from string theory". This claim has not been supported by independent studies. However, this argument has been used as one of the motivations for the model with path integral duality that we will discuss in Section 4.7. The interesting thing to note here is that the minimal length that appears in this model is not determined by the Planck length, but by the radius of the compactified dimensions. It is worth emphasizing that this approach is manifestly Lorentz invariant.

\subsection{Loop Quantum Gravity and Loop Quantum Cosmology}

Loop Quantum Gravity (LQG) is a quantization of gravity by help of carefully constructed suitable variables for quantization, variables that have become known as the Ashtekar variables [39]. While LQG theory still lacks experimental confirmation, during the last two decades it has blossomed into an established research area. Here we will only roughly sketch the main idea to see how it entails a minimal length scale. For technical details, the interested reader is referred to the more specialized reviews [42, 304, 305, 229, 118].

Since one wants to work with the Hamiltonian framework, one begins with the familiar $3+1$ split of spacetime. That is, one assumes that spacetime has topology $\mathbb{R} \times \Sigma$, i.e., it can be sliced into a set of spacelike 3-dimensional hypersurfaces. Then, the metric can be parameterized with the lapse-function $N$ and the shift vector $N_{i}$

$$
\mathrm{d} s^{2}=\left(N^{2}-N_{a} N^{a}\right) \mathrm{d} t^{2}-2 N_{a} \mathrm{~d} t \mathrm{~d} x-q_{a b} \mathrm{~d} x^{a} \mathrm{~d} x^{b}
$$

where $q_{i j}$ is the three metric on the slice. The three metric by itself does not suffice to completely describe the four dimensional spacetime. If one wants to stick with quantities that make sense on the three dimensional surfaces, in order to prepare for quantization, one needs in addition the 'extrinsic curvature' that describes how the metric changes along the slicing

$$
K_{a b}=\frac{1}{2 N}\left(\nabla_{k} N_{i}+\nabla_{i} N_{k}-\partial_{t} q_{i j}\right),
$$

where $\nabla$ is the covariant three-derivative on the slice. So far, one is used to that from general relativity.

Next we introduce the triad or dreibein, $E_{i}^{a}$, which is a set of three vector fields

$$
q^{a b}=E_{i}^{a} E_{j}^{b} \delta^{i j} .
$$


The triad converts the spatial indices $a, b$ (small, Latin, from the beginning of the alphabet) to a locally-flat metric with indices $i, j$ (small, Latin, from the middle of the alphabet). The densitized triad

$$
\tilde{E}_{i}^{a}=\sqrt{\operatorname{det} q} E_{i}^{a},
$$

is the first set of variables used for quantization. The other set of variables is an $\mathrm{su}(2)$ connection $A_{a}^{i}$, which is related to the connection on the manifold and the external curvature by

$$
A_{a}^{i}=\Gamma_{a}^{i}+\beta K_{a}^{i},
$$

where the $i$ is the internal index and $\Gamma_{a}^{i}=\Gamma_{a j k} \epsilon^{j k i}$ is the spin-connection. The dimensionless constant $\beta$ is the 'Barbero-Immirzi parameter'. Its value can be fixed by requiring the black-hole entropy to match with the semi-classical case, and comes out to be of order one.

From the triads one can reconstruct the internal metric, and from $A$ and the triad, one can reconstruct the extrinsic curvature and thus one has a full description of spacetime. The reason for this somewhat cumbersome reformulation of general relativity is that these variables do not only recast gravity as a gauge theory, but are also canonically conjugated in the classical theory

$$
\left\{A_{a}^{i}(x), \tilde{E}_{j}^{b}(y)\right\}=\beta \delta_{a}^{b} \delta_{j}^{i} \delta^{3}(x-y),
$$

which makes them good candidates for quantization. And so, under quantization one promotes $A$ and $E$ to operators $\hat{A}$ and $\hat{E}$ and replaces the Poisson bracket with commutators,

$$
\left[\hat{A}_{b}^{j}(x), \hat{\tilde{E}}_{i}^{a}(y)\right]=\mathrm{i} \beta \delta_{a}^{b} \delta_{j}^{i} \delta^{3}(x-y) .
$$

The Lagrangian of general relativity can then be rewritten in terms of the new variables, and the constraint equations can be derived.

In the so-quantized theory one can then work with different representations, like one works in quantum mechanics with the coordinate or momentum representation, just more complicated. One such representation is the loop representation, an expansion of a state in a basis of (traces of) holonomies around all possible closed loops. However, this basis is overcomplete. A more suitable basis are spin networks $\psi_{s}$. Each such spin network is a graph with vertices and edges that carry labels of the respective $\mathrm{su}(2)$ representation. In this basis, the states of LQG are then closed graphs, the edges of which are labeled by irreducible $\mathrm{su}(2)$ representations and the vertices by $\mathrm{su}(2)$ intertwiners.

The details of this approach to quantum gravity are far outside the scope of this review; for our purposes we will just note that with this quantization scheme, one can construct operators for areas and volumes, and with the expansion in the spin-network basis $\psi_{s}$, one can calculate the eigenvalues of these operators, roughly as follows.

Given a two-surface $\Sigma$ that is parameterized by two coordinates $x^{1}, x^{2}$ with the third coordinate $x^{3}=0$ on the surface, the area of the surface is

$$
A_{\Sigma}=\int_{\Sigma} \mathrm{d} x^{1} \mathrm{~d} x^{2} \sqrt{\operatorname{det} q^{(2)}},
$$

where $\operatorname{det} q^{(2)}=q_{11} q_{22}-q_{12}^{2}$ is the metric determinant on the surface. In terms of the triad, this can be written as

$$
A_{\Sigma}=\int_{\Sigma} \mathrm{d} x^{1} \mathrm{~d} x^{2} \sqrt{\tilde{E}_{i}^{3} \tilde{E}^{3 i}} .
$$

This area can be promoted to an operator, essentially by making the triads operators, though to deal with the square root of a product of these operators one has to average the operators over 
smearing functions and take the limit of these smearing functions to delta functions. One can then act with the so-constructed operator on the states of the spin network and obtain the eigenvalues

$$
\hat{A}_{\Sigma} \psi_{s}=8 \pi l_{\mathrm{Pl}}^{2} \beta \sum_{I} \sqrt{j_{I}\left(j_{I}+1\right)} \psi_{s},
$$

where the sum is taken over all edges of the network that pierce the surface $\Sigma$, and $j_{I}$, a positive half-integer, are the representation labels on the edge. This way, one finds that LQG has a minimum area of

$$
A_{\min }=4 \pi \sqrt{3} \beta l_{\mathrm{Pl}}^{2} .
$$

A similar argument can be made for the volume operator, which also has a finite smallestpossible eigenvalue on the order of the cube of the Planck length [271, 303, 41]. These properties then lead to the following interpretation of the spin network: the edges of the graph represent quanta of area with area $\sim l_{\mathrm{P}}^{2} \sqrt{j(j+1)}$, and the vertices of the graph represent quanta of 3volume.

Loop Quantum Cosmology (LQC) is a simplified version of LQG, developed to study the time evolution of cosmological, i.e., highly-symmetric, models. The main simplification is that, rather than using the full quantized theory of gravity and then studying models with suitable symmetries, one first reduces the symmetries and then quantizes the few remaining degrees of freedom.

For the quantization of the degrees of freedom one uses techniques similar to those of the full theory. LQC is thus not strictly speaking derived from LQG, but an approximation known as the 'mini-superspace approximation.' For arguments why it is plausible to expect that LQC provides a reasonably good approximation and for a detailed treatment, the reader is referred to $[40,44,58,57,227]$. Here we will only pick out one aspect that is particularly interesting for our theme of the minimal length.

In principle, one works in LQC with operators for the triad and the connection, yet the semiclassical treatment captures the most essential features and will be sufficient for our purposes. Let us first briefly recall the normal cosmological Friedmann-Robertson-Walker model coupled to scalar field $\phi$ in the new variables [118]. The ansatz for the metric is

$$
\mathrm{d} s^{2}=-\mathrm{d} t^{2}+a^{2}(t)\left(\mathrm{d} x^{2}+\mathrm{d} y^{2}+\mathrm{d} z^{2}\right),
$$

and for the Ashtekar variables

$$
A_{a}^{i}=c \delta_{a}^{i}, \quad \tilde{E}_{i}^{a}=p \delta_{i}^{a} .
$$

The variable $p$ is dimensionless and related to the scale factor as $a^{2}=|p|$, and $c$ has dimensions of energy. $c$ and $p$ are canonically conjugate and normalized so that the Poisson brackets are

$$
\{c, p\}=\frac{8 \pi}{3} \beta .
$$

The Hamiltonian constraint for gravity coupled to a (spatially homogeneous) pressureless scalar field with canonically conjugated variables $\phi, p_{\phi}$ is

$$
16 \pi G \mathcal{H}=-\frac{6}{\beta^{2}} c^{2}|p|^{1 / 2}+8 \pi G p_{\phi}^{2}|p|^{-3 / 2}=0 .
$$

This yields

$$
\frac{c}{\beta}=2 \sqrt{\frac{\pi}{3}} l_{\mathrm{Pl}} \frac{p_{\phi}}{|p|} .
$$


Since $\phi$ itself does not appear in the Hamiltonian, the conjugated momentum $p_{\phi}$ is a constant of motion $\dot{p}_{\phi}=0$, where a dot denotes a derivative with respect to $t$. The equation of motion for $\phi$ is

$$
\dot{\phi}=\frac{p_{\phi}}{p^{3 / 2}},
$$

so we can identify

$$
\rho_{\phi}=\frac{p_{\phi}^{2}}{2|p|^{3}}
$$

as the energy density of the scalar field. With this, Equation (129) can be written in the more familiar form

$$
\dot{\rho}_{\phi}=-\frac{3}{2} \frac{\dot{p}}{p^{4}} p_{\phi}^{2}=-3 \frac{\dot{a}}{a} \rho_{\phi} .
$$

The equation of motion for $p$ is

$$
\dot{p}=-\frac{8 \pi}{3} \beta \frac{\partial \mathcal{H}}{\partial c}=2 \frac{c}{\beta}|p|^{1 / 2} .
$$

Inserting (128), this equation can be integrated to get

$$
p^{3 / 2}=2 \sqrt{3 \pi} l_{\mathrm{Pl}} p_{\phi} t .
$$

One can rewrite this equation by introducing the Hubble parameter $H=\dot{a} / a=\dot{p} /(2 p)$; then one finds

$$
H^{2}=\frac{4 \pi}{3} l_{\mathrm{Pl}}^{2} \frac{p_{\phi}^{2}}{p^{3}}=\frac{8 \pi}{3} G \rho_{\phi},
$$

which is the familiar first Friedmann equation. Together with the energy conservation (131) this fully determines the time evolution.

Now to find the Hamiltonian of LQC, one considers an elementary cell that is repeated in all spatial directions because space is homogeneous. The holonomy around a loop is then just given by $\exp (\mathrm{i} \mu c)$, where $c$ is as above the one degree of freedom in $A$, and $\mu$ is the edge length of the elementary cell. We cannot shrink this length $\mu$ to zero because the area it encompasses has a minimum value. That is the central feature of the loop quantization that one tries to capture in LQC; $\mu$ has a smallest value on the order of $\mu_{0} \sim l_{\mathrm{Pl}}$. Since one cannot shrink the loop to zero, and thus cannot take the derivative of the holonomy with respect to $\mu$, one cannot use this way to find an expression for $c$ in the so-quantized theory.

With that in mind, one can construct an effective Hamiltonian constraint from the classical Eq. (127) by replacing $c$ with $\sin \left(\mu_{0} c\right) / \mu_{0}$ to capture the periodicity of the network due to the finite size of the elementary loops. This replacement makes sense because the so-introduced operator can be expressed and interpreted in terms of holonomies. (For this, one does not have to use the sinus function in particular; any almost-periodic function would do [40], but the sinus is the easiest to deal with.) This yields

$$
16 \pi G H_{\mathrm{eff}}=-\frac{6}{\beta^{2}}|p|^{\frac{1}{2}} \frac{\sin ^{2}\left(\mu_{0} c\right)}{\mu_{0}}+8 \pi G \frac{1}{|p|^{\frac{3}{2}}} p_{\phi}^{2} .
$$

As before, the Hamiltonian constraint gives

$$
\frac{\sin \left(\mu_{0} c\right)}{\mu_{0} \beta}=2 \sqrt{\frac{\pi}{3}} l_{\mathrm{Pl}} \frac{p_{\phi}}{|p|} .
$$


And then the equation of motion in the semiclassical limit is

$$
\dot{p}=\left\{p, H_{\mathrm{eff}}\right\}=-\frac{8 \pi}{3} \beta \frac{\partial H_{\mathrm{eff}}}{\partial c}=\frac{2|p|^{\frac{1}{2}}}{\beta \mu_{0}} \sin \left(\mu_{0} c\right) \cos \left(\mu_{0} c\right) .
$$

With the previously found identification of $\rho_{\phi}$, we can bring this into a more familiar form

$$
H^{2}=\frac{\dot{p}^{2}}{4 p^{2}}=\frac{8 \pi}{3} G \rho_{\rho}\left(1-\frac{\rho_{\phi}}{\rho_{\mathrm{c}}}\right)
$$

where the critical density is

$$
\rho_{\mathrm{c}}=\frac{3}{8 \pi G \beta^{2} \mu_{0}^{2} a}
$$

The Hubble rate thus goes to zero for a finite $a$, at

$$
a^{2}=4 \pi G p_{\phi}^{2} \beta^{2} \mu_{0}^{2}
$$

at which point the time-evolution bounces without ever running into a singularity. The critical density at which this happens depends on the value of $p_{\phi}$, which here has been a free constant. It has been argued in [43], that by a more careful treatment the parameter $\mu_{0}$ depends on the canonical variables and then the critical density can be identified to be similar to the Planck density.

The semi-classical limit is clearly inappropriate when energy densities reach the Planckian regime, but the key feature of the bounce and removal of the singularity survives in the quantized case $[56,44,58,57]$. We take away from here that the canonical quantization of gravity leads to the existence of minimal areas and three-volumes, and that there are strong indications for a Planckian bound on the maximally-possible value of energy density and curvature.

\subsection{Quantized conformal fluctuations}

The following argument for the existence of a minimal length scale has been put forward by Padmanabhan [248, 247] in the context of conformally-quantized gravity. That is, we consider fluctuations of the conformal factor only and quantize them. The metric is of the form

$$
g_{\mu \nu}(x)=(1+\phi(x))^{2} \bar{g}_{\mu \nu}(x),
$$

and the action in terms of $\bar{g}$ reads

$$
S[\bar{g}, \phi]=\frac{1}{16 \pi G} \int \mathrm{d}^{4} x \sqrt{-\bar{g}}\left(\bar{R}(1+\phi(x))^{2}-2 \Lambda(1+\phi(x))^{4}-6 \partial^{\nu} \phi \partial_{\nu} \phi\right) .
$$

In flat Minkowski background with $\bar{g}_{\nu \kappa}=\eta_{\nu \kappa}$ and in a vacuum state, we then want to address the question what is the expectation value of spacetime intervals

$$
\left\langle 0\left|\mathrm{~d} s^{2}\right| 0\right\rangle=\left\langle 0\left|g_{\mu \nu}\right| 0\right\rangle \mathrm{d} x^{\mu} \mathrm{d} x^{\nu}=\left(1+\left\langle 0\left|\phi(x)^{2}\right| 0\right\rangle\right) \eta_{\mu \nu} \mathrm{d} x^{\mu} \mathrm{d} x^{\nu}
$$

Since the expectation value of $\phi(x)^{2}$ is divergent, instead of multiplying fields at the same point, one has to use covariant point-slitting to two points $x^{\nu}$ and $y^{\nu}=x^{\nu}+\mathrm{d} x^{\nu}$ and then take the limit of the two points approaching each other

$$
\left\langle 0\left|\mathrm{~d} s^{2}\right| 0\right\rangle=\lim _{\mathrm{d} x \rightarrow 0}(1+\langle 0|\phi(x) \phi(x+\mathrm{d} x)| 0\rangle) \eta_{\mu \nu} \mathrm{d} x^{\mu} \mathrm{d} x^{\nu} .
$$


Now for a flat background, the action (142) has the same functional form as a massless scalar field (up to a sign), so we can tell immediately what its Green's function looks like

$$
\langle 0|\phi(x) \phi(y)| 0\rangle=\frac{l_{\mathrm{p}}^{2}}{4 \pi^{2}} \frac{1}{(x-y)^{2}} .
$$

Thus, one can take the limit $\mathrm{d} x^{\nu} \rightarrow 0$

$$
\left\langle 0\left|\mathrm{~d} s^{2}\right| 0\right\rangle=\frac{l_{\mathrm{p}}^{2}}{4 \pi^{2}} \lim _{\mathrm{d} x \rightarrow 0} \frac{1}{(x-y)^{2}} \eta_{\mu \nu} \mathrm{d} x^{\mu} \mathrm{d} x^{\nu}=\frac{l_{\mathrm{p}}^{2}}{4 \pi^{2}} .
$$

The two-point function of the scalar fluctuation diverges and thereby counteracts the attempt to obtain a spacetime distance of length zero; instead one has a finite length on the order of the Planck length.

This argument has recently been criticized by Cunliff in [92] on the grounds that the conformal factor is not a dynamical degree of freedom in the pure Einstein-Hilbert gravity that was used in this argument. However, while the classical constraints fix the conformal fluctuations in terms of matter sources, for gravity coupled to quantized matter this does not hold. Cunliff reexamined the argument, and found that the scaling behavior of the Greens function at short distances then depends on the matter content; for normal matter content, the limit (146) still goes to zero.

\subsection{Asymptotically Safe Gravity}

String theory and LQG have in common the aim to provide a fundamental theory for space and time different from general relativity; a theory based on strings or spin networks respectively. Asymptotically Safe Gravity (ASG), on the other hand, is an attempt to make sense of gravity as a quantum field theory by addressing the perturbative non-renormalizability of the EinsteinHilbert action coupled to matter [300].

In ASG, one considers general relativity merely as an effective theory valid in the low energy regime that has to be suitably extended to high energies in order for the theory to be renormalizable and make physical sense. The Einstein-Hilbert action is then not the fundamental action that can be applied up to arbitrarily-high energy scales, but just a low-energy approximation and its perturbative non-renormalizability need not worry us. What describes gravity at energies close by and beyond the Planck scale (possibly in terms of non-metric degrees of freedom) is instead dictated by the non-perturbatively-defined renormalization flow of the theory.

To see how that works, consider a generic Lagrangian of a local field theory. The terms can be ordered by mass dimension and will come with, generally dimensionful, coupling constants $g_{i}$. One redefines these to dimensionless quantities $\tilde{g}_{i}=\lambda^{-d_{i}} g_{i}$, where $k$ is an energy scale. It is a feature of quantum field theory that the couplings will depend on the scale at which one applies the theory; this is described by the Renormalization Group (RG) flow of the theory. To make sense of the theory fundamentally, none of the dimensionless couplings should diverge.

In more detail, one postulates that the RG flow of the theory, described by a vector-field in the infinite dimensional space of all possible functionals of the metric, has a fixed point with finitely many ultra-violet (UV) attractive directions. These attractive directions correspond to "relevant" operators (in perturbation theory, those up to mass dimension 4) and span the tangent space to a finite-dimensional surface called the "UV critical surface". The requirement that the theory holds up to arbitrarily-high energies then implies that the natural world must be described by an RG trajectory lying in this surface, and originating (in the UV) from the immediate vicinity of the fixed point. If the surface has finite dimension $d$, then $d$ measurements performed at some energy $\lambda$ are enough to determine all parameters, and then the remaining (infinitely many) coordinates of the trajectory are a prediction of the theory, which can be tested against further experiments. 
In ASG the fundamental gravitational interaction is then considered asymptotically safe. This necessitates a modification of general relativity, whose exact nature is so far unknown. Importantly, this scenario does not necessarily imply that the fundamental degrees of freedom remain those of the metric at all energies. Also in ASG, the metric itself might turn out to be emergent from more fundamental degrees of freedom [261]. Various independent works have provided evidence that gravity is asymptotically safe, including studies of gravity in $2+\epsilon$ dimensions, discrete lattice simulations, and continuum functional renormalization group methods.

It is beyond the scope of this review to discuss how good this evidence for the asymptotic safety of gravity really is. The interested reader is referred to reviews specifically dedicated to the topic, for example [240, 202, 260]. For our purposes, in the following we will just assume that asymptotic safety is realized for general relativity.

To see qualitatively how gravity may become asymptotically safe, let $\lambda$ denote the RG scale. From a Wilsonian standpoint, we can refer to $\lambda$ as 'the cutoff'. As is customary in lattice theory, we can take $\lambda$ as a unit of mass and measure everything else in units of $\lambda$. In particular, we define with

$$
\tilde{G}=G \lambda^{2}
$$

the dimensionless number expressing Newton's constant in units of the cutoff. (Here and in the rest of this subsection, a tilde indicates a dimensionless quantity.) The statement that the theory has a fixed point means that $\tilde{G}$, and all other similarly-defined dimensionless coupling constants, go to finite values when $\lambda \rightarrow \infty$.

The general behavior of the running of Newton's constant can be inferred already by dimensional analysis, which suggests that the beta function of $1 / G$ has the form

$$
\lambda \frac{d}{d \lambda} \frac{1}{G}=\alpha \lambda^{2}
$$

where $\alpha$ is some constant. This expectation is supported by a number of independent calculations, showing that the leading term in the beta function has this behavior, with $\alpha>0$. Then the beta function of $\tilde{G}$ takes the form

$$
\lambda \frac{d \tilde{G}}{d \lambda}=2 \tilde{G}-\alpha \tilde{G}^{2} .
$$

This beta function has an IR attractive fixed point at $\tilde{G}=0$ and also a UV attractive nontrivial fixed point at $\tilde{G}_{*}=1 / \alpha$. The solution of the RG equation (148) is

$$
G(\lambda)^{-1}=G_{0}^{-1}+\frac{\alpha}{2} \lambda^{2},
$$

where $G_{0}$ is Newton's constant in the low energy limit. Therefore, the Planck length, $\sqrt{G}$, becomes energy dependent.

This running of Newton's constant is characterized by the existence of two very different regimes:

- If $0<\tilde{G} \ll 1$ we are in the regime of sub-Planckian energies, and the first term on the right side of Eq. (149) dominates. The solution of the flow equation is

$$
\tilde{G}(\lambda)=\tilde{G}_{0}\left(\frac{\lambda}{\lambda_{0}}\right)^{2},
$$

where $\lambda_{0}$ is some reference scale and $\tilde{G}_{0}=\tilde{G}\left(\lambda_{0}\right)$. Thus, the dimensionless Newton's constant is linear in $\lambda^{2}$, which implies that the dimensionful Newton's constant $G(\lambda)=G_{0}=l_{\mathrm{Pl}}^{2}$ is constant. This is the regime that we are all familiar with. 
- In the fixed point regime, on the other hand, the dimensionless Newton's constant $\tilde{G}=\tilde{G}_{*}$ is constant, which implies that the dimensionful Newton's constant runs according to its canonical dimension, $G(\lambda)=\tilde{G}_{*} / \lambda^{2}$, in particular it goes to zero for $\lambda \rightarrow \infty$.

One naturally expects the threshold separating these two regimes to be near the Planck scale. With the running of the RG scale, $\tilde{G}$ must go from its fixed point value at the Planck scale to very nearly zero at macroscopic scales.

At first look it might seem like ASG does not contain a minimal length scale because there is no limit to the energy by which structures can be tested. In addition, towards the fixed point regime, the gravitational interaction becomes weaker, and with it weakening the argument from thought experiments in Section 3.1.2, which relied on the distortion caused by the gravitational attraction of the test particle. It has, in fact, been argued $[51,108]$ that in ASG the formation of a black-hole horizon must not necessarily occur, and we recall that the formation of a horizon was the main spoiler for increasing the resolution in the earlier-discussed thought experiments.

However, to get the right picture one has to identify physically-meaningful quantities and a procedure to measure them, which leads to the following general argument for the occurrence of a minimal length in ASG [74, 261].

Energies have to be measured in some unit system, otherwise they are physically meaningless. To assign meaning to the limit of $\lambda \rightarrow \infty$ itself, $\lambda$ too has to be expressed in some unit of energy, for example as $\lambda \sqrt{G}$, and that unit in return has to be defined by some measurement process. In general, the unit itself will depend on the scale that is probed in any one particular experiment. The physically-meaningful energy that we can probe distances with in some interaction thus will generally not go to $\infty$ with $\lambda$. In fact, since $\sqrt{G} \rightarrow 1 / \lambda$, an energy measured in units of $\sqrt{G}$ will be bounded by the Planck energy; it will go to one in units of the Planck energy.

One may think that one could just use some system of units other than Planck units to circumvent the conclusion, but if one takes any other dimensionful coupling as a unit, one will arrive at the same conclusion if the theory is asymptotically safe. And if it is not, then it is not a fundamental theory that will break down at some finite value of energy and not allow us to take the limit $\lambda \rightarrow \infty$. As Percacci and Vacca pointed out in [261], it is essentially a tautology that an asymptotically-safe theory comes with this upper bound when measured in appropriate units.

A related argument was offered by Reuter and Schwindt [270] who carefully distinguish measurements of distances or momenta with a fixed metric from measurements with the physically-relevant metric that solves the equations of motion with the couplings evaluated at the scale $\lambda$ that is being probed in the measurement. In this case, the dependence on $\lambda$ naturally can be moved into the metric. Though they have studied a special subclass of (Euclidian) manifolds, their finding that the metric components go like $1 / \lambda^{2}$ is interesting and possibly of more general significance.

The way such a $1 / \lambda^{2}$-dependence of the metric on the scale $\lambda$ at which it is tested leads to a finite resolution is as follows. Consider a scattering process with in and outgoing particles in a space, which, in the infinite distance from the scattering region, is flat. In this limit, to good precision spacetime has the metric $g_{\kappa \nu}(\lambda \rightarrow 0)=\eta_{\kappa \nu}$. Therefore, we define the momenta of the in- and outgoing particles, as well as their sums and differences, and from them as usual the Lorentz-invariant Mandelstam variables, to be of the form $s=\eta_{\kappa \nu} p^{\kappa} p^{\nu}$. However, since the metric depends on the scale that is being tested, the physically-relevant quantities in the collision region have to be evaluated with the metric $g_{\kappa \nu}(\sqrt{s})=\eta_{\kappa \nu} m_{\mathrm{Pl}}^{2} / s$. With that one finds that the effective Mandelstam variables, and thus also the momentum transfer in the collision region, actually go to $g_{\kappa \nu}(\sqrt{s}) p^{\nu} p^{\mu}=m_{\mathrm{Pl}}^{2}$, and are bounded by the Planck scale.

This behavior can be further illuminated by considering in more detail the scattering process in an asymptotically-flat spacetime [261]. The dynamics of this process is described by some Wilsonian effective action with a suitable momentum scale $\lambda$. This action already takes into account the effective contributions of loops with momenta above the scale $\lambda$, so one may evaluate scattering 
at tree level in the effective action to gain insight into the scale-dependence. In particular, we will consider the scattering of two particles, scalars or fermions, by exchange of a graviton.

Since we want to unravel the effects of ASG, we assume the existence of a fixed point, which enters the cross sections of the scattering by virtual graviton exchange through the running of Newton's constant. The tree-level amplitude contains a factor $1 / m_{\mathrm{Pl}}$ for each vertex. In the $s$-channel, the squared amplitude for the scattering of two scalars is

$$
\left|M_{\mathrm{s}}^{2}\right|=\frac{1}{4 m_{\mathrm{Pl}}} \frac{t^{2} u^{2}}{s^{2}}
$$

and for fermions

$$
\left|M_{\mathrm{f}}^{2}\right|=\frac{1}{128 m_{\mathrm{Pl}}} \frac{t^{4}-6 t^{3} u+18 t^{2} u^{2}-6 t u^{3}+u^{4}}{s^{2}} .
$$

As one expects, the cross sections scale with the fourth power of energy over the Planck mass. In particular, if the Planck mass was a constant, the perturbative expansion would break down at energies comparable to the Planck mass. However, we now take into account that in ASG the Planck mass becomes energy dependent. For the annihilation process in the $s$-channel, it is $\sqrt{s}$, the total energy in the center-of-mass system, that encodes what scale can be probed. Thus, we replace $m_{\mathrm{Pl}}$ with $1 / \sqrt{G(s)}$. One proceeds similarly for the other channels.

From the above amplitudes the total cross section is found to be [261]

$$
\sigma_{\mathrm{s}}=\frac{s G(s)}{1920 \pi}, \quad \sigma_{\mathrm{f}}=\frac{s G(s)}{5120 \pi},
$$

for the scalars and fermions respectively. Using the running of the gravitational coupling constant (150), one sees that the cross section has a maximum at $s=2 G_{0} / \alpha$ and goes to zero when the center-of-mass energy goes to infinity. For illustration, the cross section for the scalar scattering is depicted in Figure 4 for the case with a constant Planck mass in contrast to the case where the Planck mass is energy dependent.

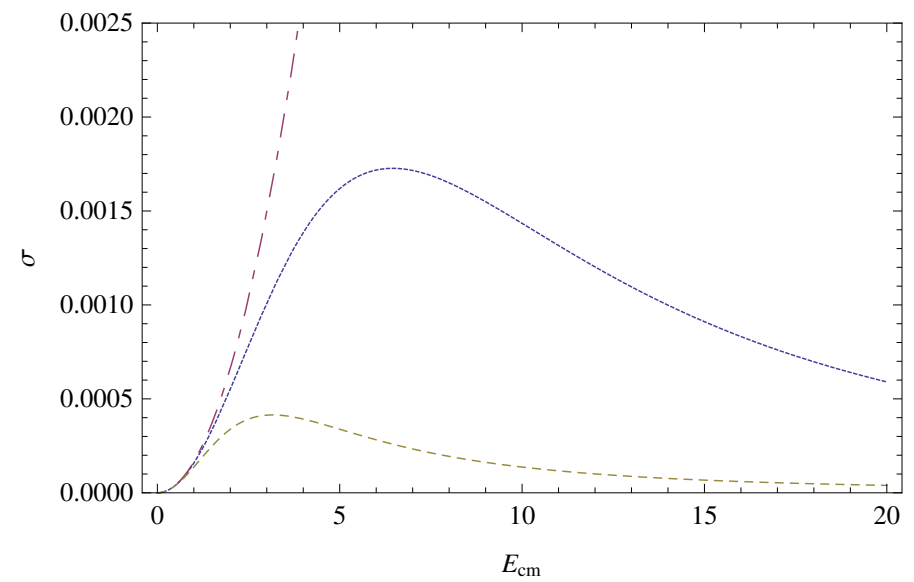

Figure 4: Cross section for scattering of two scalar particles by graviton exchange with and without running Planck mass, in units of the low-energy Planck mass $1 / \sqrt{G_{0}}$. The dot-dashed (purple) line depicts the case without asymptotic safety; the continuous (blue) and dashed (grey) line take into account the running of the Planck mass, for two different values of the fixed point, $1 / \sqrt{\tilde{G} *}=0.024$ and 0.1 respectively. Figure from [261]; reproduced with permission from IOP. 
If we follow our earlier argument and use units of the running Planck mass, then the cross section as well as the physically-relevant energy, in terms of the asymptotic quantities $G(\sqrt{s}) \sqrt{s} / G_{0}$, become constant at the Planck scale. These indications for the existence of a minimal length scale in ASG are intriguing, in particular because the dependence of the cross section on the energy offers a clean way to define a minimal length scale from observable quantities, for example through the (square root of the) cross section at its maximum value.

However, it is not obvious how the above argument should be extended to interactions in which no graviton exchange takes place. It has been argued on general grounds in [74], that even in these cases the dependence of the background on the energy of the exchange particle reduces the momentum transfer so that the interaction would not probe distances below the Planck length and cross sections would stagnate once the fixed-point regime has been reached, but the details require more study. Recently, in [30] it has been argued that it is difficult to universally define the running of the gravitational coupling because of the multitude of kinematic factors present at higher order. In the simple example that we discussed here, the dependence of $G$ on the $\sqrt{s}$ seems like a reasonable guess, but a cautionary note that this argument might not be possible to generalize is in order.

\subsection{Non-commutative geometry}

Non-commutative geometry is both a modification of quantum mechanics and quantum field theory that arises within certain approaches towards quantum gravity, and a class of theories i $n$ its own right. Thus, it could rightfully claim a place both in this section with motivations for a minimal length scale, and in Section 4 with applications. We will discuss the general idea of non-commutative geometries in the motivation because there is a large amount of excellent literature that covers the applications and phenomenology of non-commutative geometry. Thus, our treatment here will be very brief. For details, the interested reader is referred to [104, 151] and the many references therein.

String theory and M-theory are among the motivations to look at non-commutative geometries (see, e.g., the nice summary in [104], section VII) and there have been indications that LQG may give rise to a certain type of non-commutative geometry known as $\kappa$-Poincaré. This approach has been very fruitful and will be discussed in more detail later in Section 4 .

The basic ingredient to non-commutative geometry is that, upon quantization, spacetime coordinates $x^{\nu}$ are associated to Hermitian operators $\hat{x}^{\nu}$ that are non-commuting. The simplest way to do this is of the form

$$
\left[\hat{x}^{\nu}, \hat{x}^{\mu}\right]=\mathrm{i} \theta^{\mu \nu} .
$$

The real-valued, antisymmetric two-tensor $\theta_{\mu \nu}$ of dimension length squared is the deformation parameter in this modification of quantum theory, known as the Poisson tensor. In the limit $\theta_{\mu \nu} \rightarrow 0$ one obtains ordinary spacetime. In this type of non-commutative geometry, the Poisson tensor is not a dynamical field and defines a preferred frame and thereby breaks Lorentz invariance.

The deformation parameter enters here much like $\hbar$ in the commutation relation between position and momentum; its physical interpretation is that of a smallest observable area in the $\mu \nu$-plane. The above commutation relation leads to a minimal uncertainty among spacial coordinates of the form

$$
\Delta x_{\mu} \Delta x_{\nu} \gtrsim \frac{1}{2}\left|\theta^{\mu \nu}\right| .
$$

One expects the non-zero entries of $\theta_{\mu \nu}$ to be on the order of about the square of the Planck length, though strictly speaking they are free parameters that have to be constrained by experiment. 
Quantization under the assumption of a non-commutative geometry can be extended from the coordinates themselves to the algebra of functions $f(x)$ by using Weyl quantization. What one looks for is a procedure $W$ that assigns to each element $f(x)$ in the algebra of functions $\mathcal{A}$ a Hermitian operator $\hat{f}=W(f)$ in the algebra of operators $\hat{\mathcal{A}}$. One does that by choosing a suitable basis for elements of each algebra and then identifies them with each other. The most common choice $^{9}$ is to use a Fourier decomposition of the function $f(x)$

$$
\tilde{f}(k)=\frac{1}{(2 \pi)^{4}} \int \mathrm{d}^{4} x e^{-\mathrm{i} k_{\nu} x^{\nu}} f(x)
$$

and then doing the inverse transform with the non-commutative operators $\hat{x}^{\nu}$

$$
\hat{f}=W(f)=\frac{1}{(2 \pi)^{4}} \int \mathrm{d}^{4} k e^{-\mathrm{i} k_{\nu} \hat{x}^{\nu}} \tilde{f}(k) .
$$

One can extend this isomorphism between the vector spaces to an algebra isomorphism by constructing a new product, denoted $\star$, that respects the map $W$,

$$
W(f \star g)(x)=W(f) \cdot W(g)=\hat{f} \cdot \hat{g},
$$

for $f, g \in \mathcal{A}$ and $\hat{f}, \hat{g} \in \hat{\mathcal{A}}$. From Eqs. (158) and (159) one finds the explicit expression

$$
W(f \star g)=\frac{1}{(2 \pi)^{4}} \int \mathrm{d}^{4} k \mathrm{~d}^{4} p e^{\mathrm{i} k_{\nu} \hat{x}^{\nu}} e^{\mathrm{i} p_{\nu} \hat{x}^{\nu}} \tilde{f}(k) \tilde{g}(p) .
$$

With the Campbell-Baker-Hausdorff formula

$$
e^{A} e^{B}=e^{A+B+\frac{1}{2}[A, B]+\frac{1}{12}[A,[A, B]]-\frac{1}{12}[B,[A, B]]-\frac{1}{24}[B,[A,[A, B]]]+\ldots}
$$

one has

$$
e^{\mathrm{i} k_{\nu} \hat{x}^{\nu}} e^{\mathrm{i} p_{\nu} \hat{x}^{\nu}}=e^{\mathrm{i}\left(k_{\nu}+p_{\nu}\right) \hat{x}^{\nu}-\frac{\mathrm{i}}{2} k_{\nu} \theta^{\nu \kappa} p_{\kappa}},
$$

and thus

$$
W(f \star g)=\frac{1}{(2 \pi)^{4}} \int \mathrm{d}^{4} k \mathrm{~d}^{4} p e^{\mathrm{i}\left(k_{\nu}+p_{\nu}\right) \hat{x}^{\nu}-\frac{\mathrm{i}}{2} k_{\nu} \theta^{\nu \kappa} p_{\kappa}} \tilde{f}(k) \tilde{g}(p) .
$$

This map can be inverted to

$$
f(x) \star g(x)=\int \frac{\mathrm{d}^{4} p}{(2 \pi)^{4}} \frac{\mathrm{d}^{4} k}{(2 \pi)^{4}} \tilde{f}(k) \tilde{g}(p) e^{-\frac{\mathrm{i}}{2} k_{\kappa} \theta^{\kappa \nu} p_{\nu}} e^{-\mathrm{i}\left(k_{\kappa}+p_{\kappa}\right) x^{\kappa}} .
$$

If one rewrites the $\theta$-dependent factor into a differential operator acting on the plane-wave-basis, one can also express this in the form

$$
f(x) \star g(x)=\left.\exp \left(\frac{\mathrm{i}}{2} \frac{\partial}{\partial x^{\nu}} \theta^{\nu \kappa} \frac{\partial}{\partial x^{\kappa}}\right) f(x) g(y)\right|_{x \rightarrow y},
$$

which is known as the Moyal-Weyl product [236].

The star product is a particularly useful way to handle non-commutative geometries, because one can continue to work with ordinary functions, one just has to keep in mind that they obey a

9 An example of a different choice of basis can be found in [314]. 
modified product rule in the algebra. With that, one can build non-commutative quantum field theories by replacing normal products of fields in the Lagrangian with the star products.

To gain some insight into the way this product modifies the physics, it is useful to compute the star product with a delta function. For that, we rewrite Eq. (164) as

$$
\begin{aligned}
f(x) \star g(x) & =\int \frac{\mathrm{d}^{4} p}{(2 \pi)^{4}} \mathrm{~d}^{4} y f\left(x+\frac{1}{2} \theta k\right) g(x+y) e^{-\mathrm{i} k_{\kappa} y^{\kappa}} \\
& =\frac{1}{\pi^{4}|\operatorname{det} \theta|} \int \mathrm{d}^{4} z \mathrm{~d}^{4} y f(x+z) g(x+y) e^{-2 \mathrm{i} z^{\nu} \theta_{\nu \kappa}^{-1} y^{\kappa}} .
\end{aligned}
$$

And so, one finds the star product with a delta function to be

$$
\delta(x) \star g(x)=\frac{1}{\pi^{4}|\operatorname{det} \theta|} \int \mathrm{d}^{4} y e^{2 \mathrm{i} x^{\nu} \theta_{\nu \kappa}^{-1} y^{\kappa}} g(y) .
$$

In contrast to the normal product of functions, this describes a highly non-local operation. This non-locality, which is a characteristic property of the star product, is the most relevant feature of non-commutative geometry.

It is clear that the non-vanishing commutator by itself already introduces some notion of fundamentally-finite resolution, but there is another way to see how a minimal length comes into play in non-commutative geometry. To see that, we look at a Gaussian centered around zero. Gaussian distributions are of interest not only because they are widely used field configurations, but, for example, also because they may describe solitonic solutions in a potential [137].

For simplicity, we will consider only two spatial dimensions and spatial commutativity, so then we have

$$
\left[\hat{x}^{i}, \hat{x}^{j}\right]=\mathrm{i} \theta \epsilon^{i j},
$$

where $i, j \in\{1,2\}, \epsilon^{i j}$ is the totally antisymmetric tensor, and $\theta$ is the one remaining free parameter in the Poisson tensor. This is a greatly simplified scenario, but it will suffice here.

A normalized Gaussian in position space centered around zero with covariance $\sigma$

$$
\Psi_{\sigma}(x)=\frac{1}{\pi \sigma} \exp \left(-\frac{x^{2}}{\sigma^{2}}\right)
$$

has the Fourier transform

$$
\tilde{\Psi}_{\sigma}(k)=\int d^{2} x e^{i k x} \Psi_{\sigma}(x)=\exp \left(-\pi^{2} k^{2} \sigma^{2}\right) .
$$

We can then work out the star product for two Gaussians with two different spreads $\sigma_{1}$ and $\sigma_{2}$ to

$$
\begin{aligned}
\tilde{\Psi}_{\sigma_{1}} \star \tilde{\Psi}_{\sigma_{2}}(k) & =\int d^{2} k \Psi_{\sigma_{1}}(k) \Psi_{\sigma_{2}}(p-k) \exp \left(\frac{\mathrm{i}}{2} k^{i} \epsilon_{i j} p^{j}\right) \\
& =\frac{\pi}{\left(4 \sigma_{1}^{2}+\sigma_{2}^{2}\right)^{2}} \exp \left(-\frac{p^{2} \sigma_{12}^{2}}{4}\right)
\end{aligned}
$$

where

$$
\sigma_{12}^{2}=\frac{\sigma_{1}^{2} \sigma_{2}^{2}+\theta^{2}}{\sigma_{1}^{2}+\sigma_{2}^{2}}
$$

Back in position space this yields

$$
\Psi_{\sigma_{1}} \star \tilde{\Psi}_{\sigma_{2}}(x)=\frac{1}{\pi \sigma_{12}\left(4 \sigma_{1}^{2}+\sigma_{2}^{2}\right)^{2}} \exp \left(-\frac{x^{2}}{\sigma_{12}^{2}}\right) .
$$


Thus, if we multiply two Gaussians with $\sigma_{1}, \sigma_{2}<\theta$, the width of the product $\sigma_{12}$ is larger than $\theta$. In fact, if we insert $\sigma_{1}=\sigma_{2}=\sigma_{12}=\sigma$ in Eq. (172) and solve for $\sigma$, we see that a Gaussian with width $\sigma=\theta$ squares to itself. Thus, since Gaussians with smaller width than $\theta$ have the effect to spread, rather than to focus, the product, one can think of the Gaussian with width $\theta$ as having a minimum effective size.

In non-commutative quantum mechanics, even in more than one dimension, Gaussians with this property constitute solutions to polynomial potentials with a mass term (for example for a cubic potential this would be of the form $\left.V(\phi)=m^{2} \phi \star \phi+a_{2} \phi \star \phi \star \phi \star \phi\right)$ [137], because they square to themselves, and so only higher powers continue to reproduce the original function.

\subsection{Miscellaneous}

Besides the candidate theories for quantum gravity so far discussed, there are also discrete approaches, reviewed, for example, in [203]. For these approaches, no general statement can be made with respect to the notion of a minimal length scale. Though one has lattice parameters that play the role of regulators, the goal is to eventually let the lattice spacing go to zero, leaving open the question of whether observables in this limit allow an arbitrarily good resolution of structures or whether the resolution remains bounded. One example of a discrete approach, where a minimal length appears, is the lattice approach by Greensite [139] (discussed also in Garay [120]), in which the minimal length scale appears for much the same reason as it appears in the case of quantized conformal metric fluctuations discussed in Section 3.4. Even if the lattice spacing does not go to zero, it has been argued on general grounds in [60] that discreteness does not necessarily imply a lower bound on the resolution of spatial distances.

One discrete approach in which a minimal length scale makes itself noticeable in yet another way are Causal Sets [290]. In this approach, one considers as fundamental the causal structure of spacetime, as realized by a partially-ordered, locally-finite set of points. This set, represented by a discrete sprinkling of points, replaces the smooth background manifold of general relativity. The "Hauptvermutung" (main conjecture) of the Causal Sets approach is that a causal set uniquely determines the macroscopic (coarse-grained) spacetime manifold. In full generality, this conjecture is so far unproven, though it has been proven in a limiting case [63]. Intriguingly, the causal sets approach to a discrete spacetime can preserve Lorentz invariance. This can be achieved by using not a regular but a random sprinkling of points; there is thus no meaningful lattice parameter in the ordinary sense. It has been shown in [62], that a Poisson process fulfills the desired property. This sprinkling has a finite density, which is in principle a parameter, but is usually assumed to be on the order of the Planckian density.

Another broad class of approaches to quantum gravity that we have so far not mentioned are emergent gravity scenarios, reviewed in [49, 284]. Also in these cases, there is no general statement that can be made about the existence of a minimal length scale. Since gravity is considered to be emergent (or induced), there has to enter some energy scale at which the true fundamental, non-gravitational, degrees of freedom make themselves noticeable. Yet, from this alone we do not know whether this also prevents a resolution of structures. In fact, in the absence of anything resembling spacetime, this might not even be a meaningful question to ask.

Giddings and Lippert [128, 129, 126] have proposed that the gravitational obstruction to test short distance probes should be translated into a fundamental limitation in quantum gravity distinct from the GUP. Instead of a modification of the uncertainty principle, fundamental limitations should arise due to strong gravitational (or other) dynamics, because the concept of locality is only approximate, giving rise to a 'locality bound' beyond which the notion of locality ceases to be meaningful. When the locality bound is violated, the usual field theory description of matter no longer accurately describes the quantum state and one loses the rationale for the usual Fock space description of the states; instead, one would have to deal with states able to describe a quantum 
black hole, whose full and proper quantum description is presently unknown.

Finally, we should mention an interesting recent approach by Dvali et al. that takes very seriously the previously-found bounds on the resolution of structures by black-hole formation [106] and is partly related to the locality bound. However, rather than identifying a regime where quantum field theory breaks down and asking what quantum theory of gravity would allow one to consistently deal with strong curvature regimes, in Dvali et al.'s approach of 'classicalization', super-Planckian degrees of freedom cannot exist. On these grounds, it has been argued that classical gravity is in this sense UV-complete exactly because an arbitrarily good resolution of structures is physically impossible [105].

\subsection{Summary of motivations}

In this section we have seen that there are many indications, from thought experiments as well as from different approaches to quantum gravity, that lead us to believe in a fundamental limit to the resolution of structure. But we have also seen that these limits appear in different forms.

The most commonly known form is a lower bound on spatial and temporal resolutions given by the Planck length, often realized by means of a GUP, in which the spatial uncertainty increases with the increase of the energy used to probe the structures. Such an uncertainty has been found in string theory, but we have also seen that this uncertainty does not seem to hold in string theory in general. Instead, in this particular approach to quantum gravity, it is more generally a spacetime uncertainty that still seems to hold. One also has to keep in mind here that this bound is given by the string scale, which may differ from the Planck scale. LQG and the simplified model for LQC give rise to bounds on the eigenvalues of the area and volume operator, and limit the curvature in the early universe to a Planckian value.

Thus, due to these different types of bounds, it is somewhat misleading to speak of a 'minimal length,' since in many cases a bound on the length itself does not exist, but only on the powers of spatio-temporal distances. Therefore, it is preferable to speak more generally of a 'minimal length scale,' and leave open the question of how this scale enters into the measurement of physical quantities. 


\section{Models and Applications}

In this section we will investigate some models that have been developed to deal with a minimal length or, more often, a maximum energy scale. The models discussed in the following are not in themselves approaches to a fundamental description of spacetime like the ones previously discussed that lead us to seriously consider a finite resolution of structures. Instead, the models discussed in this section are attempts to incorporate the notion of a minimal length into the standard model of particle physics and/or general relativity by means of a modification of quantum mechanics, quantum field theory and Poincaré symmetry. These models are meant to provide an effective description of the possible effects of a minimal length with the intention to make contact with phenomenology and thereby ideally constrain the possible modifications. ${ }^{10}$

As mentioned previously, the non-commutative geometries discussed in Section 3.6 could also have rightfully claimed a place in this section on models and applications.

Before we turn towards the implementation, let us spend some words on the interpretation because the construction of a suitable model depends on the physical picture one aims to realize.

\subsection{Interpretation of a minimal length scale}

It is the central premise of this review that there exists a minimal length scale that plays a fundamental role in the laws of nature. In the discussion in Section 5 we will consider the possibility that this premise is not fulfilled, but for now we try to incorporate a minimal length scale into the physical description of the world. There are then still different ways to think about a minimal length scale or a maximum energy scale.

One perspective that has been put forward in the literature [14, 193, 15, 212] is that for the Planck mass to be observer independent it should be invariant under Lorentz boosts. Since normal Lorentz boosts do not allow this, the observer independence of the Planck mass is taken as a motivation to modify special relativity to what has become known as 'Deformed Special Relativity' (DSR). (See also Section 4.5). In brief, this modification of special relativity allows one to perform a Lorentz boost on momentum space in such a way that an energy of Planck mass remains invariant.

While that is a plausible motivation to look into such departures from special relativity, one has to keep in mind that just because a quantity is of dimension length (mass) it must not necessarily transform under Lorentz boosts as a spatial or time-like component of a spacetime (or momentum) four vector. A constant of dimension length can be invariant under normal Lorentz boosts, for example, if it is a spacetime (proper) distance. This interpretation is an essential ingredient to Padmanabhan's path integral approach (see Section 4.7). Another example is the actual mass of a particle, which is invariant under Lorentz boosts by merit of being a scalar. Thinking back to our historical introduction, we recall that the coupling constant of Fermi's theory for the weak interaction is proportional to the inverse of the $W$-mass and therefore observer independent in the sense that it does not depend on the rest frame in which we determine it - and that without the need to modify special relativity.

Note also that coupling constants do depend on the energy with which structures are probed, as discussed in Section 3.5 on ASG. There we have yet another interpretation for a minimal length scale, that being the energy range (in terms of normally Lorentz-invariant Mandelstam variables for the asymptotically in/out-going states) where the running Planck mass comes into the fixed-point regime. The center-of-mass energy corresponding to the turning point of the total cross section in graviton scattering, for example, makes it a clean and observer-independent definition of an energy scale, beyond which there is no more new structure to be found.

10 The word 'effective' should here not be read as a technical term. 
One should also keep in mind that the outcome of a Lorentz boost is not an observable per se. To actually determine a distance in some reference frame one has to perform a measurement. Thus, for the observer independence of a minimal length, it is sufficient if there is no operational procedure that allows one to resolve structures to a precision better than the Planck length. It has been argued in [154] that this does not necessitate a modification of Lorentz boosts for the momenta of free particles; it is sufficient if the interactions of particles do not allow one to resolve structures beyond the Planck scale. There are different ways this could be realized, for example, by an off-shell modification of the propagator that prevents arbitrarily-high momentum transfer. As previously discussed, there are some indications that ASG might realize such a feature and it can, in a restricted sense, be interpreted as a version of DSR [74].

An entirely different possibility that we mentioned in Section 3.7, has recently been put forward in $[106,105]$, where it was argued that it is exactly because of the universality of black-hole production that the Planck length already plays a fundamental role in classical gravity and there is no need to complete the theory in the high energy range.

\subsection{Modified commutation relations}

The most widely pursued approach to model the effects of a minimal length scale in quantum mechanics and quantum field theory is to reproduce the GUP starting from a modified commutation relation for position and momentum operators. This modification may or may not come with a modification also of the commutators of these operators with each other, which would mean that the geometry in position or momentum space becomes non-commuting.

The modified commutation relations imply not only a GUP, but also a modified dispersion relation and a modified Poincaré-symmetry in momentum and/or position space. The literature on the subject is vast, but the picture is still incomplete and under construction.

\subsubsection{Recovering the minimal length from modified commutation relations}

To see the general idea, let us start with a simple example that shows the relation of modified commutation relations to the minimal length scale. Consider variables $\mathbf{k}=(\vec{k}, \omega)$, where $\vec{k}$ is the three vector, components of which will be labeled with small Latin indices, and $\mathbf{x}=(\vec{x}, t)$. Under quantization, the associated operators obey the standard commutation relations

$$
\left[x^{\nu}, x^{\kappa}\right]=0, \quad\left[x^{\nu}, k_{\kappa}\right]=\mathrm{i} \delta_{\kappa}^{\nu} \quad, \quad\left[k_{\nu}, k_{\kappa}\right]=0 .
$$

Now we define a new quantity $\mathbf{p}=(\vec{p}, E)=f(\mathbf{k})$, where $f$ is an injective function, so that $f^{-1}(\mathbf{p})=$ $\mathbf{k}$ is well defined. We will also use the notation $p_{\mu}=h_{\mu}^{\alpha}(\mathbf{k}) k_{\alpha}$ with the inverse $k_{\alpha}=h_{\alpha}^{\mu}(\mathbf{p}) p_{\mu}$. For the variables $\mathbf{x}$ and $\mathbf{p}$ one then has the commutation relations

$$
\left[x^{\nu}, x^{\kappa}\right]=0, \quad\left[x^{\nu}, p_{\kappa}\right]=\mathrm{i} \frac{\partial f_{\kappa}}{\partial k_{\nu}}, \quad\left[p_{\nu}, p_{\kappa}\right]=0 .
$$

If one now looks at the uncertainty relation between $x^{i}$ and $p_{i}$, one finds

$$
\Delta x_{i} \Delta p_{i} \geq \frac{1}{2}\left\langle\frac{\partial f_{i}}{\partial k^{i}}\right\rangle .
$$

To be concrete, let us insert some function, for example a generic expansion of the form $\vec{p} \approx$ $\vec{k}\left(1+\alpha k^{2} / m_{\mathrm{Pl}}^{2}\right)$ plus higher orders in $k / m_{\mathrm{Pl}}$, so that the inverse relation is $\vec{k} \approx \vec{p}\left(1-\alpha p^{2} / m_{\mathrm{Pl}}^{2}\right)$. Here, $\alpha$ is some dimensionless parameter. One then has

$$
\frac{\partial f_{i}}{\partial k^{j}} \approx \delta_{i j}\left(1+\alpha \frac{p^{2}}{m_{\mathrm{Pl}}^{2}}\right)+2 \alpha \frac{p_{i} p_{j}}{m_{\mathrm{Pl}}^{2}} .
$$


Since this function is convex, we can rewrite the expectation value in (176) to

$$
\Delta x_{i} \Delta p_{i} \geq \frac{1}{2}\left(1+\alpha \frac{\left\langle p^{2}\right\rangle}{m_{\mathrm{Pl}}^{2}}+2 \alpha \frac{\left\langle p_{i}^{2}\right\rangle}{m_{\mathrm{Pl}}^{2}}\right) .
$$

And inserting the expression for the variance $\left\langle A^{2}\right\rangle-\langle A\rangle^{2}=\Delta A^{2}$, one obtains

$$
\begin{aligned}
\Delta x_{i} \Delta p_{i} & \geq \frac{1}{2}\left(1+\alpha \frac{\Delta p^{2}+\langle p\rangle^{2}}{m_{\mathrm{Pl}}^{2}}+2 \alpha \frac{\Delta p_{i}^{2}+\left\langle p_{i}\right\rangle^{2}}{m_{\mathrm{Pl}}^{2}}\right) \\
& \geq \frac{1}{2}\left(1+3 \alpha \frac{\Delta p_{i}^{2}}{m_{\mathrm{Pl}}^{2}}\right),
\end{aligned}
$$

or

$$
\Delta x_{i} \geq \frac{1}{2}\left(\frac{1}{\Delta p_{i}}+3 \alpha \frac{\Delta p_{i}}{m_{\mathrm{Pl}}^{2}}\right) .
$$

Thus, we have reproduced the GUP that we found in the thought experiments in Section 3.1 with a minimal possible uncertainty for the position [175, 26, 207, 209, 25].

However, though the nomenclature here is deliberately suggestive, one has to be careful with interpreting this finding. What the inequality (180) tells us is that we cannot measure the position to arbitrary precision if we do it by varying the uncertainty in $\mathbf{p}$. That has an operational meaning only if we assign to $\mathbf{p}$ the meaning of a physical momentum, in particular it should be a Hermitian operator. To distinguish between the physical quantity $\mathbf{p}$, and $\mathbf{k}$ that fulfills the canonical commutation relations, the $\mathbf{k}$ is sometimes referred to as the 'pseudo-momentum' or, because it is conjugated to $\mathbf{x}$, as 'the wave vector.' One can then physically interpret the non-linear relation between $\mathbf{p}$ and $\mathbf{k}$ as an energy dependence of Planck's constant [156].

To further clarify this, let us turn towards the question of Lorentz invariance. If we do not make statements in addition to the commutation relations, we do not know anything about the transformation behavior of the quantities. For all we know, they could have an arbitrary transformation behavior and Lorentz invariance could just be broken. If we require Lorentz invariance to be preserved, this opens the question of how it is preserved, what is the geometry of its phase space, and what is its Poisson structure. And, most importantly, how do we identify physically-meaningful coordinates on that space?

At the time of writing, no agreed upon picture has emerged. Normally, phase space is the cotangential bundle of the spacetime manifold. One might generalize this to a bundle of curved momentum spaces, an idea that dates back at least to Max Born in 1938 [64]. In a more radical recent approach, the 'principle of relative locality' [287, 286, 169, 27, 23, 21] phase space is instead considered to be the cotangential bundle of momentum space.

To close this gap in our example, let us add some more structure and assume that the phase space is a trivial bundle $\mathcal{S}=\mathcal{M} \otimes \mathcal{P}$, where $\mathcal{M}$ is spacetime and $\mathcal{P}$ is momentum space. Elements of this space have the form $(\mathbf{x}, \mathbf{p})$. If we add that the quantity $\mathbf{p}$ is a coordinate on $\mathcal{P}$ and transforms according to normal Lorentz transformations under a change of inertial frames, and $\mathbf{k}$ is another coordinate system, then we know right away how $\mathbf{k}$ transforms because we can express it by use of the function $f$. If we do a Lorentz transformation on $\mathbf{p}$, so that $\mathbf{p}^{\prime}=\Lambda \mathbf{p}$, then we have

$$
\mathbf{k}^{\prime}=f\left(\mathbf{p}^{\prime}\right)=f(\Lambda \mathbf{p})=f\left(\Lambda f^{-1}(\mathbf{k})\right),
$$

which we can use to construct the modified Lorentz transformation as $\mathbf{k}^{\prime}=\widetilde{\Lambda}(\mathbf{k})$. In particular, one can chose $f$ in such a way that it maps an infinite value of $\mathbf{p}$ (in either the spatial or temporal entries, or both) to a finite value of $\mathbf{k}$ at the Planck energy. The so-constructed Lorentz transformation on p will then keep the Planck scale invariant, importantly without introducing any preferred frame. 
This is the basic idea of deformations of special relativity, some explicit examples of which we will meet in Section 4.5.

If one assumes that $\mathbf{p}$ transforms as a normal Lorentz vector, one has

$$
\left[J_{\kappa \nu}, p_{\mu}\right]=\mathrm{i}\left(p_{\nu} \eta_{\kappa \mu}-p_{\kappa} \eta_{\nu \mu}\right) .
$$

Since this commutator commutes with $\mathbf{p}$, one readily finds

$$
\left[J_{\kappa \nu}, k_{\mu}\right]=\mathrm{i}\left(p_{\nu} \eta_{\kappa \alpha}-p_{\kappa} \eta_{\nu \alpha}\right) \frac{\partial k_{\mu}}{\partial p_{\alpha}},
$$

which gives us the infinitesimal version of (181) by help of the usual expansion

$$
k^{\prime}{ }_{\nu}=k_{\nu}-\frac{\mathrm{i}}{2} \omega^{\alpha \beta}\left[J_{\alpha \beta}, k_{\nu}\right]+\mathcal{O}\left(\omega^{2}\right),
$$

where $\omega^{\alpha \beta}$ are the group parameters of $\Lambda$.

Now that we know how $\mathbf{k}$ transforms, we still need to add information for how the coordinates on $\mathcal{P}$ are supposed to be matched to those on $\mathcal{M}$. One requirement that we can use to select a basis on $\mathcal{M}$ along with that on $\mathcal{P}$ under a Lorentz transformation is that the canonical form of the commutation relations should remain preserved. With this requirement, one then finds for the infinitesimal transformation of $\mathbf{x}[253]$

$$
\left[J_{\kappa \nu}, x^{\mu}\right]=\mathrm{i} x^{\alpha} \frac{\partial}{\partial k_{\mu}}\left(p_{\kappa} \eta_{\beta \nu} \frac{\partial k_{\alpha}}{\partial p_{\beta}}-p_{\nu} \eta_{\beta \kappa} \frac{\partial k_{\alpha}}{\partial p_{\kappa}}\right)
$$

and the finite transformation

$$
x^{\prime \nu}=\frac{\partial p_{\alpha}^{\prime}}{\partial k_{\mu}^{\prime}} \Lambda_{\alpha}^{\nu} \frac{\partial k_{\beta}}{\partial p_{\nu}} x^{\kappa} .
$$

One finds the latter also directly by noting that this transformation behavior is required to keep the symplectic form $w=d x^{\alpha} \wedge d k_{\alpha}$ canonical.

A word of caution is in order here because the innocent looking indices on these quantities do now implicitly stand for different transformation behaviors under Lorentz transformations. One can amend this possible confusion by a more complicated notation, but this is for practical purposes usually unnecessary, as long as one keeps in mind that the index itself does not tell the transformation behavior under Lorentz transformations. In particular, the derivative $\partial k_{\alpha} / \partial p_{\beta}$ has a mixed transformation behavior and, in a Taylor-series expansion, this and higher derivatives yield factors that convert the normal to the modified transformation behavior.

So far this might have seemed like a rewriting, so it is important to stress the following: just writing down the commutation relation leaves the structure under-determined. To completely specify the model, one needs to make an additional assumption about how a Lorentz transformation is defined, how the coordinates in position space ought to be chosen along with those in momentum space under a Lorentz transformation and, most importantly, what the metric on the curved momentum space (and possibly spacetime) is.

Needless to say, the way we have fixed the transformation behavior in this simple example is not the only way to do it. Another widely used choice is to require that $\mathbf{k}$ obeys the usual transformation behavior, yet interpret $\mathbf{p}$ as the physical momentum, or $\mathbf{x}$ as 'pseudo-coordinates' (though this is not a word that has been used in the literature). We will meet an example of this in Section 4.2.2. This variety is the main reason why the literature on the subject of modified commutation relations is confusing.

At this point it should be mentioned that a function $f$ that maps an infinite value of $p$ to an asymptotically-finite value of $f(p)$ cannot be a polynomial of finite order. Its Taylor series 
expansion necessarily needs to have an infinite number of terms. Now if $k(p)$ becomes constant for large $p$, then $\partial k / \partial p$ goes to zero and $\partial p / \partial k$ increases without bound, which is why the uncertainty (176) increases for large $p$. Depending on the choice of $f$, this might be the case for the spatial or temporal components or both. If one wants to capture the regularizing properties of the minimal length, then a perturbative expansion in powers of $E / m_{\mathrm{Pl}}$ will not work in the high energy limit. In addition, such expansions generically add the complication that any truncation of the series produces for the dispersion relation a polynomial of finite order, which will have additional zeros, necessitating additional initial values [158]. This can be prevented by not truncating the series, but this adds other complications, discussed in Section 4.4.

Since $\partial p / \partial k$ is a function of $\mathbf{p}$, the position operator $\mathrm{i}\left(\partial p_{\alpha} / \partial k_{\nu}\right) \partial / \partial p_{\alpha}$, is not Hermitian if $\mathbf{p}$ is Hermitian. This is unfortunate for a quantity that is supposed to be a physical observable, but we have to keep in mind that the operator itself is not an observable anyway. To obtain an observable, we have to take an expectation value. To ensure that the expectation value produces meaningful results, we change its evaluation so that the condition $\langle x \Psi \mid \Phi\rangle=\langle\Psi \mid x \Phi\rangle$, which in particular guarantees that expectation values are real, is still fulfilled. That is, we want the operator to be symmetric, rather than Hermitian. ${ }^{11}$ To that end, one changes the measure in momentum space to $[175,184]$

$$
\mathrm{d}^{3} p \rightarrow \mathrm{d}^{3} k=\left|\frac{\partial k}{\partial p}\right| \mathrm{d}^{3} p
$$

which will exactly cancel the non-Hermitian factor in $\hat{x}=\mathrm{i} \partial /(\partial k)$, because now

$$
\begin{aligned}
\langle\Psi \mid x \Phi\rangle & =\mathrm{i} \int \mathrm{d}^{3} p\left|\frac{\partial k}{\partial p}\right| \Psi^{*} \frac{\partial}{\partial k} \Phi \\
& =\mathrm{i} \int \mathrm{d}^{3} k \Psi^{*} \frac{\partial}{\partial k} \Phi=\mathrm{i} \int \mathrm{d}^{3} k\left(\frac{\partial}{\partial k} \Psi^{*}\right) \Phi \\
& =\langle x \Psi \mid \Phi\rangle .
\end{aligned}
$$

Note that this does not work without the additional factor because then the integration measure does not fit to the derivative, which is a consequence of the modified commutation relations. We will see in the next section that there is another way to think of this modified measure.

The mass-shell relation, $g^{\mu \nu}(\mathbf{p}) p_{\mu} p_{\nu}-m^{2}=0$, is invariant under normal Lorentz transformations acting on $\mathbf{p}$, and thus $g^{\mu \nu}(\mathbf{k}) p_{\mu}(\mathbf{k}) p_{\nu}(\mathbf{k})^{2}-m^{2}=0$ is invariant under the modified Lorentz transformations acting on $\mathbf{k}$. The clumsy notation here has stressed that the metric on momentum space will generally be a non-linear function of the coordinates. The mass-shell relation will yield the Hamiltonian constraint of the theory.

There is a subtlety here; since $\mathbf{x}$ is not Hermitian, we can't use the representation if this operator. The way this can be addressed depends on the model. One can in many cases just work the momentum representation. In our example, we would note that the operator $\bar{x}^{\nu}=\left(\partial k_{\nu} / \partial p_{\mu}\right) x^{\mu}$ is Hermitian, and use its representation. In this representation, the Hamiltonian constraint becomes a higher-order operator, and thus delivers a modification of the dispersion relation. However, the interpretation of the dispersion then hinges on the interpretation of the coordinates. Depending on the suitable identification of position space coordinates and the function $f$, the speed of massless particles in this model may thus become a function of the momentum four-vector $\mathbf{p}$. It should be noted however that this is not the case for all choices of $f$ [155].

Many of the applications of this model that we will meet later only use the first or second-order expansion of $f$. While these are sufficient for some interesting consequences of the GUP, the

11 According to the Hellinger-Toeplitz theorem, an everywhere-defined symmetric operator on a Hilbert space is necessarily bounded. Since some operators in quantum mechanics are unbounded, one is required to deal with wave functions that are not square integrable. The same consideration applies here. 
Planck energy is then generically not an asymptotic and invariant value. If the complete function $f$ is considered, it is usually referred to as an 'all order GUP.' The expansion to first or second order is often helpful because it allows one to parameterize the possible modifications by only a few dimensionless quantities.

To restrict the form of modifications possible in this approach, sometimes the Jacobi identities are drawn upon. It is true that the Jacobi identities restrict the possible commutation relations, but seen as we did here starting from the standard commutation relations, this statement is somewhat misleading. The Jacobi identities are, as the name says, identities. They say more about the properties of the binary operation they represent than about the quantities this operation acts on. They are trivially fulfilled for the commutators of all new variables $f(k)$ one can define (coordinates one can choose) if the old ones fulfilled the identities. However, if one does not start from such a function, one can draw upon the Jacobi identities as a consistency check.

A requirement that does put a restriction on the possible form of the commutation relation is that of rotational invariance. Assuming that $f_{i}(\vec{k})=k_{i} h(k)$, where $k=|\vec{k}|$, one has

$$
\frac{\partial f_{i}}{\partial k_{j}}=\delta_{i}^{j} h(k)+k_{i} \frac{\partial h(k)}{\partial k_{j}} .
$$

The expansion to 3 rd order in $k$ is

$$
h(k)=1+\alpha k+\beta k^{2}+\mathcal{O}\left(k^{3}\right), \quad \frac{\partial h}{\partial k_{j}}=\alpha \frac{k_{j}}{k}+2 \beta k_{j}+\mathcal{O}\left(k^{2}\right) .
$$

We denote the inverse of $f_{i}(\vec{k})$ with $f_{i}^{-1}(\vec{p})=p_{i} \tilde{h}(p)$. An expansion of $\tilde{h}(p)$ and comparison of coefficients yields to third order

$$
\tilde{h}(p)=1-\alpha p-\left(\beta-2 \alpha^{2}\right) p^{2} .
$$

With this, one has then

$$
\begin{aligned}
{\left[x_{i}, p_{j}\right] } & =\delta_{i j}+\alpha\left(k \delta_{i j}+\frac{k_{i} k_{j}}{k}\right)+\beta\left(k^{2} \delta_{i j}+2 k_{j} k_{i}\right)+\mathcal{O}\left(k^{3}\right) \\
& =\delta_{i j}+\alpha\left(p \delta_{i j}+\frac{p_{i} p_{j}}{p}\right)+\left(\beta-\alpha^{2}\right) p^{2} \delta_{i j}+\left(2 \beta-\alpha^{2}\right) p_{i} p_{j}+\mathcal{O}\left(p^{3}\right) .
\end{aligned}
$$

For the dimensions to match, the constant $\alpha$ must have a dimension of length and $\beta$ a dimension of length squared. One would expect this length to be of the order Planck length and play the role of the fundamental length.

It is often assumed that $\beta=\alpha^{2}$, but it should be noted that this does not follow from the above. In particular, $\alpha$ may be zero and the modification be even in $k$, starting only at second order. Note that an expansion of the commutator in the form $\left[x_{i}, p_{j}\right]=\mathrm{i} \delta_{i j}\left(1+\beta p^{2}\right)$ does not fulfill the above requirement.

To summarize this section, we have seen that a GUP that gives rise to a minimal length scale can be realized by modifying the canonical commutation relations. We have seen that this modification alone does not completely specify the physical picture, we have in addition to fix the transformation behavior under Lorentz transformations and the metric on momentum space. In Section 4.2.2 we will see how this can be done.

\subsubsection{The Snyder basis}

As mentioned in the previous example, $\mathbf{p}$ is not canonically conjugate to $\mathbf{x}$, and the wave vector $\mathbf{k}$, which is canonically conjugate, is the quantity that transforms under the modified Lorentz 
transformations. But that is not necessarily the case for models with modified commutation relations, as we will see in this section.

Let us start again from the normal commutation relations (174) and now define new position coordinates $\mathbf{X}$ by $X_{\nu}=x_{\nu}-x^{\alpha} k_{\alpha} k_{\nu} / m_{\mathrm{Pl}}^{2}$, as discussed in [135]. (The Planck mass $m_{\mathrm{Pl}}$ could enter here with an additional dimensionless factor that one would expect to be of order one, if one describes a modification that has its origin in quantum gravitational effects. In the following we will not carry around such an additional factor. It can easily be inserted at any stage just by replacing $m_{\mathrm{Pl}}$ with $\alpha m_{\mathrm{Pl}}$.) In addition, we now require that $\mathbf{k}$ transforms under the normal Lorentz transformations. With this replacement, the k's are then still commutative as usual and the remaining commutation relations have the form

$$
\left[X_{\mu}, X_{\nu}\right]=-\frac{1}{m_{\mathrm{Pl}}} J_{\mu \nu}, \quad\left[X_{\mu}, k_{\nu}\right]=i\left(\eta_{\mu \nu}-\frac{k_{\mu} k_{\nu}}{m_{\mathrm{Pl}}^{2}}\right),
$$

where we recognize

$$
J_{\mu \nu}=x_{\mu} k_{\nu}-x_{\nu} k_{\mu}=X_{\mu} k_{\nu}-X_{\nu} k_{\mu}
$$

as the generators of Lorentz transformations. This reproduces the commutation relations of Snyder's original proposal [288].

The commutator between $\mathbf{X}$ and $\mathbf{k}$ leads to a GUP by taking the expectation value in the same way as previously, though the reason here is a different one: If it is the $X_{\nu}$ 's that are representing physically-meaningful positions in spacetime, then it is their non-commutativity that spoils the resolution of structures at the Planck scale. Note that the transformation from $\mathbf{x}$ to $\mathbf{X}$ is not canonical exactly for the reason that it does change the commutation relations.

In a commonly-used notation, $J_{i 0}=N_{i}$ are the generators of boosts and $\left\{J_{23}, J_{31}, J_{12}\right\}$ are the generators of the rotations $\left\{M_{1}, M_{2}, M_{3}\right\}$ that fulfill the normal Lorentz algebra

$$
\begin{aligned}
{\left[N_{i}, N_{j}\right] } & =-i \epsilon_{i j k} N_{k}, \quad\left[M_{i}, M_{j}\right]=i \epsilon_{i j k} N_{k}, \\
{\left[M_{i}, N_{j}\right] } & =i \epsilon_{i j k} N_{k} .
\end{aligned}
$$

Since we have not done anything to the transformation of the momentum $\mathbf{k}$, in the $\mathbf{X}, \mathbf{k}$ phase-space coordinates one also has

$$
\begin{aligned}
& {\left[M_{i}, k_{j}\right]=i \epsilon_{i j k} k_{k}, \quad\left[M_{i}, k_{0}\right]=0,} \\
& {\left[N_{i}, k_{j}\right]=i \delta_{i j} k_{0}, \quad\left[N_{i}, k_{0}\right]=i k_{i} .}
\end{aligned}
$$

There are two notable things here. First, as in Section 4.2.2, the Lorentz algebra remains entirely unmodified. Second, the $\mathbf{X}$ by construction transforms covariantly under normal Lorentz transformations if the $\mathbf{x}$ and $\mathbf{k}$ do. However, we see that there is exactly one $\mathbf{x}$ for which $\mathbf{X}$ does not depend on $\mathbf{k}$, and that is $\mathbf{x}=0$. If we perform a translation by use of the generator $\mathbf{k}$; the coordinate $\mathbf{x}$ will be shifted to some value $\mathbf{x}^{\prime}=\mathbf{x}+\mathbf{a}$. Alternatively, one may try to take a different generator for translations than $\mathbf{k}$, the obvious choice is the operator canonically conjugated to $X_{\nu}$

$$
\frac{\partial}{\partial X_{\nu}}=\frac{\partial}{\partial x_{\alpha}} \frac{\partial x_{\alpha}}{\partial X_{\nu}}
$$

If one contracts $X_{\nu}=x_{\nu}+x^{\alpha} k_{\alpha} k_{\nu} / m_{\mathrm{Pl}}^{2}$ with $k^{\nu}$, one can invert $\mathbf{X}(\mathbf{x})$ to

$$
x_{\nu}=X_{\nu}-\frac{X_{\alpha} k^{\alpha}}{m_{\mathrm{Pl}}^{2}+k_{\kappa} k^{\kappa}} k_{\nu} .
$$


Then one finds the translation operator

$$
\frac{\partial}{\partial X_{\nu}}=k^{\nu}\left(\frac{1}{1-k^{\alpha} k_{\alpha} / m_{\mathrm{Pl}}^{2}}\right) .
$$

Therefore, it has been argued [230] that one should understand this type of theory as a modification of translation invariance rather than a modification of Lorentz symmetry. However, this depends on which variables are assigned physical meaning, which is a question that is still under discussion.

We should at this point look at Snyder's original motivation for it is richer than just the commutation relations of position, momenta and generators and adds to it in an important way. Snyder originally considered a 5 -dimensional flat space of momenta, in which he looked at a hypersurface with de Sitter geometry. The full metric has the line element

$$
\mathrm{d} s^{2}=\eta^{A B} \mathrm{~d} \eta_{A} \mathrm{~d} \eta_{B},
$$

where the coordinates $\eta_{A}$ have dimensions of energy and capital Latin indices run from 0 to 4 . This flat space is invariant under the action of the group $S O(4,1)$, which has a total of 10 generators. In that 5 -dimensional space, consider a 4 -dimensional hyperboloid defined by

$$
-m_{p}^{2}=\eta^{A B} \eta_{A} \eta_{B}=\eta^{\mu \nu} \eta_{\mu} \eta_{\nu}-\eta_{4}^{2} .
$$

This hypersurface is invariant under the $S O(3,1)$ subgroup of $S O(4,1)$. It describes a de Sitter space and can be parameterized by four coordinates. Snyder chooses the projective coordinates $k_{\nu}=m_{\mathrm{Pl}} \eta_{\nu} / \eta_{4}$. (These coordinates are nowadays rarely used to parameterize de Sitter space, as the fifth coordinate of the embedding space $\eta_{4}$ is not constant on the hyperboloid.) The remaining four generators of $S O(4,1)$ are then identified with the coordinates

$$
J_{4 \nu}=X_{\nu}=i\left(\frac{\eta_{4}}{m_{\mathrm{Pl}}} \frac{\partial}{\partial \eta_{\nu}}+\frac{\eta_{\nu}}{m_{\mathrm{Pl}}} \frac{\partial}{\partial \eta_{4}}\right) .
$$

From this one obtains the same commutation relations (193), (195), and (196) as above [55].

However, the Snyder approach contains additional information: We know that the commutation relations seen previously can be obtained by a variable substitution from the normal ones. In addition, we know that the momentum space is curved. It has a de Sitter geometry, a non-trivial curvature tensor and curvature scalar $12 / m_{\mathrm{Pl}}^{2}$. It has the corresponding parallel transport and a volume measure. In these coordinates, the line element has the form

$$
\mathrm{d} s^{2}=\frac{\eta^{\mu \nu} \mathrm{d} k_{\mu} \mathrm{d} k_{\nu}}{1-\eta^{\alpha \kappa} k_{\alpha} k_{\kappa} / m_{\mathrm{Pl}}^{2}} .
$$

Thus, we see how the previously found need to adjust the measure in momentum space (187) arises here naturally from the geometry in momentum space. The mass-shell condition is

$$
m^{2}=\frac{\eta^{\mu \nu} k_{\mu} k_{\nu}}{1-\eta^{\alpha \kappa} k_{\alpha} k_{\kappa} / m_{\mathrm{Pl}}^{2}} .
$$

We note that on-shell this amounts to a redefinition of the rest mass.

The $(\mathbf{X}, \mathbf{k})$ coordinates on phase space have become known as the Snyder basis.

\subsubsection{The choice of basis in phase space}

The coordinates $\boldsymbol{\eta}$ that Snyder chose to parameterize the hyperbolic 4-dimensional submanifold are not unique. There are infinitely many sets of coordinates we can choose on this space; most of 
them will be non-linear combinations of each other. Such non-linear redefinitions of momenta will change the commutation relations between position and momentum variables. More generally, the question that arises here is what coordinates on phase space should be chosen, since we have seen in the previous Section 4.2.2 that a change of coordinates in phase space that mixes position and momentum operators creates non-commutativity. For example, one could use a transformation that mixes $\mathbf{p}$ and $\mathbf{x}$ to absorb the unusual factor in the $[\mathbf{x}, \mathbf{p}]$ commutator in (175) at the expense of creating a non-commutative momentum space.

Besides the above-discussed coordinate systems $(\mathbf{x}, \mathbf{k}),(\mathbf{x}, \mathbf{p}),(\mathbf{X}, \boldsymbol{\eta})$, and $(\mathbf{X}, \mathbf{k})$, there are various other choices of coordinates that can be found in the literature. One choice that is very common are coordinates $\tilde{\mathbf{x}}$ that are related to the Snyder position variables [197] via

$$
\tilde{x}_{0}=X_{0}, \quad \tilde{x}_{i}=X_{i}+\frac{N_{i}}{m_{\mathrm{p}}} .
$$

This leads to the commutation relations

$$
\left[\tilde{x}_{0}, \tilde{x}_{i}\right]=-\mathrm{i} \frac{x_{i}}{m_{\mathrm{Pl}}}, \quad\left[\tilde{x}_{i}, \tilde{x}_{j}\right]=0 .
$$

The non-commutative spacetime described by these coordinates has become known as $\kappa$-Minkowski spacetime. The name derives from the common nomenclature in which the constant $m_{\mathrm{Pl}}$ (that, as we have warned previously, might differ from the actual Planck mass by a factor of order one) is $\kappa$.

Another choice of coordinates that can be found in the literature $[132,287]$ is obtained by the transformation

$$
\chi_{0}=x_{0}+x_{i} k^{i} / m_{\mathrm{Pl}}, \quad \chi_{i}=x_{i}
$$

on the normal coordinates $x_{\nu}$. This leads to the commutation relations

$$
\begin{aligned}
& {\left[\chi_{0}, \chi_{i}\right]=\mathrm{i} \frac{\chi_{i}}{m_{\mathrm{Pl}}}, \quad\left[\chi_{i}, \chi_{j}\right]=0, \quad\left[k_{i}, \chi_{j}\right]=\mathrm{i} \delta_{i j},} \\
& {\left[\chi_{0}, k_{0}\right]=\mathrm{i}, \quad\left[\chi_{0}, k_{i}\right]=-\mathrm{i} \frac{k_{i}}{m_{\mathrm{Pl}}}, \quad\left[k_{0}, \chi_{i}\right]=\mathrm{i} .}
\end{aligned}
$$

This is the full $\kappa$-Minkowski phase space [205], which is noteworthy because it was shown by Kowalski-Glikman and Nowak [197] that the geometric approach to phase space is equivalent to the algebraic approach that has been pursued by deforming the Poincaré-algebra (the algebra of generators of Poincaré transformations, i.e., boosts, rotations and momenta) to a Hopf algebra [213] with deformation parameter $\kappa$, the $\kappa$-Poincaré Hopf algebra, giving rise to the above $\kappa$-Minkowski phase space.

A Hopf algebra generally consists of two algebras that are related by a dual structure and associated product rules that have to fulfill certain compatibility conditions. Here, the dual to the $\kappa$ Poincaré algebra is the $\kappa$-Poincaré group, whose elements are identified as Lorentz transformations and position variables. The additional structure that we found in the geometric approach to be the curvature of momentum space is, in the algebraic approach, expressed in the co-products and antipodes of the Hopf algebra. As in the geometrical approach, there is an ambiguity in the choice of coordinates in phase space.

In addition to the various choices of position space coordinates, one can also use different coordinates on momentum space, by choosing different parameterizations of the hypersurface than that of Snyder. One such parameterization is using coordinates $\pi_{\nu}$, that are related to the Snyder 
basis by

$$
\begin{aligned}
\eta_{0} & =-m_{\mathrm{Pl}} \sinh \left(\frac{\pi_{0}}{m_{\mathrm{Pl}}}\right)-\frac{\vec{\pi}^{2}}{2 m_{\mathrm{Pl}}} \exp \left(\frac{\pi_{0}}{m_{\mathrm{Pl}}}\right), \\
\eta_{i} & =-\pi_{i} \exp \left(\frac{\pi_{0}}{m_{\mathrm{Pl}}}\right), \\
\eta_{4} & =-m_{\mathrm{Pl}} \cosh \left(\frac{\pi_{0}}{m_{\mathrm{Pl}}}\right)-\frac{\vec{\pi}^{2}}{2 m_{\mathrm{Pl}}} \exp \left(\frac{\pi_{0}}{m_{\mathrm{Pl}}}\right) .
\end{aligned}
$$

(Recall that $\eta_{4}$ is not constant on the hypersurface.) The $\pi_{\nu}$ 's are the bicrossproduct basis of the Hopf algebra [213], and they make a natural choice for the algebraic approach. With the $\kappa$-Minkowski coordinates $\tilde{x}_{\mu}$, one then has the commutators [197]

$$
\begin{aligned}
& {\left[\pi_{0}, \tilde{x}_{0}\right]=\mathrm{i}, \quad\left[\pi_{i}, \tilde{x}_{0}\right]=-\mathrm{i} \frac{\pi_{i}}{m_{\mathrm{Pl}}},} \\
& {\left[\pi_{i}, \tilde{x}_{j}\right]=-\mathrm{i} \delta_{i j}, \quad\left[\pi_{0}, \tilde{x}_{i}\right]=0 .}
\end{aligned}
$$

Another choice of coordinates on momentum space is the Magueijo-Smolin basis $\mathcal{P}_{\mu}$, which is related to the Snyder coordinates by

$$
k_{\mu}=\frac{\mathcal{P}_{\mu}}{1-\mathcal{P}_{0} / m_{\mathrm{Pl}}} .
$$

From the transformation behavior of the $\mathbf{k}$ (196), one can work out the transformation behavior of the other coordinates, and reexpress the mass-shell condition 204 in the new sets of coordinates.

Since there are infinitely many other choices of coordinates, listing them all is beyond the scope of this review. So long as one can identify a new set of coordinates by a coordinate transformation from other coordinates, the commutation relations will fulfill the Jacobi identities automatically. Thus, these coordinate systems are consistent choices. One can also, starting from the transformation of the Snyder coordinates, derive the transformation behavior under Lorentz transformation for all other sets of coordinates. For the above examples the transformation behavior can be found in $[132,197]$

In summary, we have seen here that there are many different choices of coordinates on phase space that lead to modified commutation relations. We have met some oft used examples and seen that the most relevant information is in the geometry of momentum space. Whether there are particular choices of coordinates on phase space that lend themselves to easy interpretations and are thus natural in some sense is presently an open question.

\subsubsection{Multi-particle states}

One important consequence of the modified Lorentz symmetry that has been left out in our discussion so far is the additivity of momenta, which becomes relevant when considering interactions.

In the example from Section 4.2.1, the function $f$ has to be non-linear to allow a maximum value of (some components of) $\mathbf{k}$ to remain Lorentz invariant, and consequently the Lorentz transformations $\widetilde{\Lambda}$ are non-linear functions of $\mathbf{k}$. But that means that the transformation of a sum of pseudo-momenta $\mathbf{k}_{1}+\mathbf{k}_{2}$ is not the same as the sum of the transformations:

$$
\widetilde{\Lambda}\left(\mathbf{k}_{1}+\mathbf{k}_{2}\right) \neq \widetilde{\Lambda}\left(\mathbf{k}_{1}\right)+\widetilde{\Lambda}\left(\mathbf{k}_{2}\right) .
$$

Now in the case discussed in Section 4.2.1, it is the $\mathbf{p}$ that is the physical momentum that is conserved, and since it transforms under normal Lorentz transformations its conservation is independent of the rest frame. 
However, if one has chosen the $\mathbf{p}$ rather than the $\mathbf{k}$ to obey the normal Lorentz transformation, as was the case in Sections 4.2.2 and 4.2.3, then this equation looks exactly the other way round

$$
\widetilde{\Lambda}\left(\mathbf{p}_{1}+\mathbf{p}_{2}\right) \neq \widetilde{\Lambda}\left(\mathbf{p}_{1}\right)+\widetilde{\Lambda}\left(\mathbf{p}_{2}\right) .
$$

And now one has a problem, because the momentum $\mathbf{p}$ should be conserved in interactions, and the above sum is supposed to be conserved in an interaction in one rest frame, it would not be conserved generally in all rest frames. (For the free particle, both $\mathbf{p}$ and $\mathbf{k}$ are conserved since the one is a function of the other.)

The solution to this problem is to define a new, non-linear, addition law $\oplus$ that has the property that it remains invariant and that can be rightfully interpreted as a conserved quantity. This is straightforward to do if we once again use the quantities $\mathbf{k}$ that in this case by assumption transform under the normal Lorentz transformation. To each momentum we have an associated pseudo-momentum $\mathbf{k}_{1}=f^{-1}\left(\mathbf{p}_{1}\right), \mathbf{k}_{2}=f^{-1}\left(\mathbf{p}_{2}\right)$. The sum $\mathbf{k}_{1}+\mathbf{k}_{2}$ is invariant under normal Lorentz transformations, and so we construct the sum of the $\mathbf{p}^{\prime} s$ as

$$
\mathbf{p}_{1} \oplus \mathbf{p}_{2}=f\left(\mathbf{k}_{1}+\mathbf{k}_{2}\right)=f\left(f^{-1}\left(\mathbf{p}_{1}\right)+f^{-1}\left(\mathbf{p}_{2}\right)\right) .
$$

It is worthwhile to note that this modified addition law can also be found from the algebraic approach; it is the bicrossproduct of the $\kappa$-Poincare algebra [197].

This new definition for a sum is now observer independent by construction, but we have created a new problem. If the function $f$ (or some of its components) has a maximum of the Planck mass, then the sum of momenta will never exceed this maximal energy. The Planck mass is a large energy as far as particle physics is concerned, but in everyday units it is about $10^{-5}$ gram, a value that is easily exceeded by some large molecules. This problem of reproducing a sensible multi-particle limit when one chooses the physical momentum to transform under modified Lorentz transformations has become known as the 'soccer-ball problem.'

The soccer-ball problem is sometimes formulated in a somewhat different form. If one makes an expansion of the function $f$ to include the first correction terms in $p / m_{\mathrm{Pl}}$, and from that derives the sum $\oplus$, then it remains to be shown that the correction terms stay small if one calculates sums over a large number of momenta, whose ordinary sum describes macroscopic objects like, for example, a soccer ball. One expects that the sum then has approximately the form $\mathbf{p}_{1} \oplus \mathbf{p}_{2} \approx$ $\mathbf{p}_{1}+\mathbf{p}_{2}+\mathbf{p}_{1} \mathbf{p}_{2} \Gamma / m_{\mathrm{Pl}}$, where $\Gamma$ are some coefficients of order one. If one iterates this sum for $N$ terms, the normal sum grows with $N$ but the additional term with $\sim N^{2}$, so that it will eventually become problematic.

Note that this problem is primarily about sums of momenta, and not even necessarily about bound states. If one does not symmetrize the new addition rule, the result may also depend on the order in which momenta are added. This means in particular the sum of two momenta can depend on a third term that may describe a completely unrelated (and arbitrarily far away) part of the universe, which has been dubbed the 'spectator problem' [195, 134].

There have been various attempts to address the problem, but so far none has been generally accepted. For example, it has been suggested that with the addition of $N$ particles, the Planck scale that appears in the Lorentz transformation, as well as in the modified addition law, should be rescaled to $m_{\mathrm{Pl}} N[212,171,210]$. It is presently difficult to see how this ad-hoc solution would follow from the theory. Alternatively, it has been suggested that the scaling of modifications should go with the density $[157,246]$ rather than with the total momentum or energy respectively. While the energy of macroscopic objects is larger than that of microscopic ones, the energy density decreases instead. This seems a natural solution to the issue but would necessitate a completely different ansatz to implement. A noteworthy recent result is [24, 166], where it has been shown how the problem can be alleviated in a certain model, such that the nonlinear term in the sum does not go with $N^{2}$ but with $N^{3 / 2}$. 
One should also note that this problem does not occur in the case in which the modification is present only off-shell, which seems to be suggested in some interpretations. Then, if one identifies the momenta of particles as those of the asymptotically free states, the addition of their momenta is linear as usual. For the same reason, the problem also does not appear in the interpretation of such modifications of conservation laws as being caused by a running Planck's constant, put forward [261, 74], and discussed in Section 3.5. In this case, the relevant energy is the momentum transfer, and for bound states this remains small if the total mass increases.

So we have seen that demanding the physical momentum rather than the pseudo-momentum to transform under modified Lorentz transformations leads to the soccer ball and the spectator problem. This is a disadvantage of this choice. On the other hand, this choice has the advantage that it has a geometric base, which is missing for the case discussed in Section 4.2.1.

\subsubsection{Open problems}

We have, in Section 4.2.2 and 4.2.3, seen different examples for modified commutation relations with a curved momentum space. But commutation relations alone don't make for physics. To derive physical meaning, one has to define the dynamics of the system and its observables.

This raises the question of which principles to use for the dynamics and how to construct observables. While there are several approaches to this, some of which we will meet in the following, there exists to date no agreed-upon framework by which to derive observables, and therefore the question of whether there is a physical reason to prefer one basis over another is open.

One finds some statements in the literature that a different choice of coordinates leads to a different physics, but this statement is somewhat misleading. One should more precisely say that a choice of coordinates and the corresponding commutation relations do not in and of themselves determine the physics. For that, one has to specify not only the geometry of the phase space, which is not contained merely in the commutation relations, but also a unique procedure to arrive at equations of motion.

Given the complete geometry (or, equivalently, the operations on the Hopf algebra), the Hamiltonian in some basis can be identified as (a function of) the Casimir operator of the Lorentz group. ${ }^{12}$ It can be expressed in any basis one wishes by substitution. However, if the transformation between one basis of phase space and the other is not canonical, then transforming the Hamiltonian by substitution will not preserve the Hamiltonian equations. In particular, $\partial H / \partial p$ and $[H, x]$ will generically not yield the same result, thus the notion of velocity requires careful interpretation, especially when the coordinates in position space are in addition non-commuting. It has been argued by Smolin in [286] that commuting coordinates are the sensible choice. The construction of observables with non-commuting coordinates has been worked towards, e.g., in [287, 286, 169, 27, 23, 21, 272, 302, 136].

The one modification that all of these approaches have in common is a non-trivial measure in momentum space that in the geometric approach results from the volume element of the now curved space. But this raises the question of what determines the geometry. Ideally one would like an axiomatic approach that allows one to derive the geometry from an underlying principle, and then everything else from the geometry. One would, in the general case, if dynamical matter distributions are present, not expect the structure of momentum space to be entirely fixed. A step towards a dynamical momentum space has been made in [86], but clearly the topic requires more investigation.

Another open problem with this class of models is the type of non-locality that arises. If the Planck length acts as a minimal length, there clearly has to be some non-locality. However, it has been shown that for those types of models in which the speed of light becomes energy dependent,

\footnotetext{
12 The Lorentz group has a second Casimir operator, which is the length of the Pauli-Lubanski pseudovector. It can be identified by it being a function of the angular momentum operator.
} 
the non-locality becomes macroscopically large. Serious conceptual problems arising from this were pointed out in [16, 277, 156], and shown to be incompatible with observation in [159, 160].

A very recent development to address this problem is to abandon an absolute notion of locality, and instead settle for a relative one. This 'principle of relative locality' [287, 286, 169, 27, 23, 21, 79] is a promising development. It remains to be seen how it mitigates the problem of non-local particle interactions. For some discussion, see [162, 163, 161]. It should be stressed that this problem does not occur if the speed of light remains constant for free particles.

\subsection{Quantum mechanics with a minimal length scale}

So we have seen that modified commutation relations necessarily go together with a GUP, a modified measure in momentum space and a modified Lorentz symmetry. These models may or may not give rise to a modified on-shell dispersion relation and thus an energy-dependent speed of light [155], but the modified commutation relations and the generalized uncertainty cannot be treated consistently without taking care of the momentum space integration and the transformation behavior.

The literature on the subject of quantum mechanics with a minimal length scale is partly confusing because many models use only some of the previously-discussed ingredients and do not subscribe to all of the modifications, or at least they are not explicitly stated. Some differ in the interpretation of the quantities; notoriously there is the question of what is a physically-meaningful definition of velocity and what is the observable momentum.

Thus, the topic of a minimal length scale has thus given rise to many related approaches that run under the names 'modified commutation relation,' 'generalized uncertainty,' 'deformed special relativity,' 'minimal length deformed quantum mechanics,' etc. and are based on only some features of the modified phase space discussed previously. It is not clear in all cases whether this is consistently possible or what justifies a particular interpretation. For example, one may argue that in the non-relativistic limit, a modified transformation behavior under boosts, that would only become relevant at relativistic energies, is irrelevant. However, one has to keep in mind that the non-linear transformation behavior of momenta results in a non-linear addition law, which becomes problematic for the treatment of multi-particle states. Thus, even in the non-relativistic case, one has to be careful if one deals with a large number of particles.

The lack of clean, agreed upon, axiomatic approach has inevitably given rise to occasional criticism. It has been argued in [5], for example, that the deformations of special relativity are operationally indistinguishable from special relativity. Such misunderstandings are bound to arise if the model is underspecified. Maybe, the easiest way to see that the minimal length modified quantum theory is not equivalent to the unmodified case is to keep in mind that the momentum space is curved: There is no coordinate transformation that will make the curvature of momentum space go away. The non-trivial metric will also produce an infinite series of higher-order derivatives in the Hamiltonian constraint, a reflection of the non-locality that the existence of a minimal length scale implies.

In the following, we will not advocate one particular approach, but just report what results are presently available. Depending on which quantities are raised to physical importance, the resulting model can have very different properties. The speed of light might be energy dependent [26] or not [301, 99], there might be an upper limit to energies and/or momenta, or not, addition laws and thresholds might be modified, or not, coordinates might be non-commuting, or not, there might be non-localities or not, the modification might only be present off-shell, or not. This is why the physical meaning of different bases in phase space is a problem in need of being addressed in order to arrive at more stringent predictions. 


\subsubsection{Maximal localization states}

The most basic information about the minimal length modified quantum mechanics is in the position operator itself. While, in the momentum representation, there exist eigenvectors of the position operator that correspond to arbitrarily-sharply-peaked wave functions, these do not describe physically-possible configurations. It has been shown in [184] that the sharply-peaked wavefunctions with spread below the minimal position uncertainty carry an infinite energy, and thus do not represent a physically-meaningful basis. Instead, one can construct quasi-localized states that are as sharply focused as physically possible. These states are then no longer exactly orthogonal. In [184], the maximal localization states have been constructed in one spatial dimension for a 2nd-order expansion of the GUP.

\subsubsection{The Schrödinger equation with potential}

The most straight-forward modification of quantum mechanics that one can construct with the modified commutation relations is leaving the Hamiltonian unmodified. For the harmonic oscillator for example, one then has the familiar expression

$$
H=\frac{\mathbf{p}^{2}}{2 m}+m \omega^{2} \frac{\mathbf{x}^{2}}{2} .
$$

However, due to the modified commutation relations, if one inserts the operators, the resulting differential equation becomes higher order. In one dimension, for example, in the momentum space representation, one would have to second order $\hat{x}=\mathrm{i}\left(1+l_{\mathrm{Pl}}^{2} p^{2}\right) \partial / \partial p$ and thus for the stationary equation

$$
\frac{\partial^{2}}{\partial p^{2}} \psi(p)+\frac{2 l_{\mathrm{Pl}}}{1+l_{\mathrm{Pl}}^{2} p^{2}} \frac{\partial}{\partial p} \Psi(p)+\frac{2 E /\left(2 m \omega^{2}\right)-p^{2} /(m \omega)^{2}}{\left(1+l_{\mathrm{Pl}}^{2} p^{2}\right)^{2}} \psi(p)=0 .
$$

The same procedure can be applied to other types of potentials in the Schrödinger equation, and in principle this can be done not only in the small-momentum expansion, but to all orders. In this fashion, in the leading-order approximation, the harmonic oscillator in one dimension has been studied in [184, 167, 8, 125], the harmonic oscillator in arbitrary dimensions in [83, 177, 85, 93], the energy levels of the hydrogen atom in [167, 69, 296, 67, 258], the particle in a box in [7], Landau levels and the tunneling current in [8, 96, 97], the uniform gravitational potential in [244, 83], the inverse square potential in [65, 66], neutrino oscillations in [292], reflection and transmission coefficients of a potential step and potential barrier in [8, 97], the Klein paradox in [124], and corrections to the gyromagnetic moment of the muon in [143, 95]. Note that these leading order expansions do not all use the same form of the GUP.

In order to obtain the effects of the minimal length on the transition rate of ultra cold neutrons in gravitational spectrometers, Pedram et al. calculated the quantization of the energy spectrum of a particle in a linear gravitational field in the GUP leading-order approximation [259] and to all orders [256]. The harmonic oscillator in one dimension with an asymptotic GUP has been considered in [257, 255].

While not, strictly speaking, falling into the realm of quantum mechanics, let us also mention here the Casimir effect, which has been studied in [142, 46, 241, 112, 102], and Casimir-Polder intermolecular forces, which have been looked at in [252].

All these calculations do, in principle, cause corrections to results obtained in standard quantum mechanics. As one expects, the correction terms are unobservably small if one assumes the minimal length scale to be on the order of the Planck length. However, as argued previously, since we have no good explanation as to why the Planck length as the scale at which quantum gravity should become important is so small, the minimal length should, in principle, be regarded as a free 
parameter and then be bound by experiment. A compilation of bounds from the above calculations is presently not available and unfortunately no useful comparison is possible due to the different parameterizations and assumptions used. One can hope that this might improve in the future if a more standardized approach becomes established, for example, using the parameterization (192).

\subsubsection{The Klein-Gordon and Dirac equation}

The Klein Gordon equation can be obtained directly from the invariant $\mathbf{p}(\mathbf{k})^{2}-m^{2}=0$. The Dirac equation can be constructed using the same prescription that lead to the Schrödinger equation, except that, to make sure Lorentz invariance is preserved, one should first bring it into a suitable form

$$
\left(\gamma^{\nu} p_{\nu}-m\right) \Psi=0
$$

and then replace $p_{\nu}$ with its operator as discussed in Section 4.2.1. In the position representation, this will generally produce higher-order derivatives not only in the spatial, but also in the temporal, components. In order to obtain the Hamiltonian that generates the time evolution, one then has to invert the temporal part.

The Dirac equation with modified commutation relations has been discussed in [167, 188]. The Klein-Gordon equation and the Dirac particle in a rectangular and spherical box has been examined in [98].

\subsection{Quantum field theory with a minimal length scale}

One can construct a quantum field theory along the lines of the quantum mechanical treatment, starting with the modified commutation relations. If the position-space coordinates are non-commuting with a constant Poisson tensor, this leads to the territory of non-commutative quantum field theory for which the reader is referred to the literature specialized on that topic, for example [104, 151] and the many references therein.

Quantum field theory with the $\kappa$-Poincaré algebra on the non-commuting $\kappa$-Minkowski spacetime coordinates has been pioneered in $[174,178,183]$. In [176] it has been shown that introducing the minimal length uncertainty principle into quantum field theory works as a regulator in the ultraviolet, at least for $\phi^{4}$ theory. Recently, there has been a lot of progress on the way towards field quantization [31], by developing the Moyal-Weyl product, the Fock space [75, 34], and the conserved Noether charges [113, 4, 35]. The case of scalar field theory has been investigated in [33, 226]. A different second-order modification of the commutation relation has been investigated for the spinor and Klein-Gordon field in [233] and [232] respectively. [77] studied the situation in which the Hamiltonian remains unmodified and only the equal time commutation relations are modified. There are, as yet, not many applications in the literature that investigate modifications of the standard model of particle physics, but one can expect these to follow soon.

Parallel to this has been the development of quantum field theory in the case where coordinates are commuting, the physical momentum transforms under the normal Lorentz transformation, and the speed of light is constant [167]. This approach has the advantage of being easier to interpret, yet has the disadvantage of delivering more conservative predictions. In this approach, the modifications one is left with are the modified measure in momentum space and the higherorder derivatives that one obtains from the metric in momentum space. The main difficulty in this approach is that, when one takes into account gauge invariance, one does not only obtain an infinite series of higher-derivate corrections to the propagator, one also obtains an infinite number of interaction terms. Whether these models are unitary is an open question.

In order to preserve the super-Planckian limit that is necessary to capture the presence of the UV-regulating properties, it has been suggested in [167] that one expand the Lagrangian in terms 
of $g E / m_{\mathrm{Pl}}$, where $g$ is the coupling constant and $E$ is the energy scale. This means that the corrections to the propagator (which do not contain any $g$ ) are kept entirely, but one has only the first vertex of the infinite series of interaction terms. One can then explore the interesting energy range $>m_{\mathrm{Pl}}$ until $m_{\mathrm{Pl}} / g$. The virtue of this expansion, despite its limited range of applicability, is that, by not truncating the power series of the propagator, one does not introduce additional poles. The expansion in terms of $E / m_{\mathrm{Pl}}$ was looked at in [188].

In this modified quantum field theory, in [152] the running gauge couplings, possibly with additional compactified spatial dimensions, were investigated. In [189], the electro-weak gauge interaction with minimal length was studied. In [231], the top quark phenomenology in the case with a lowered Planck scale was studied, and in [153, 81] it has been argued that if the Planck scale is indeed lowered, then its role as a minimal length would decrease the production of black holes.

One recurring theme in these models is the suppression of phase space at high energies [167], which is a direct consequence of the modified measure in momentum space. This has also been found, for the same reason, in the $\kappa$-Poincare approach [311]. Another noteworthy feature of these quantum field theories with a minimal length is that the commutator between the fields $\phi(x)$ and their canonical conjugate $\pi(y)$ are not equal to a delta function [157, 221], which is an expression of the non-locality that the higher-order derivatives bring in.

In [190] it has furthermore been suggested that one apply this modification of the quantization procedure to quantum cosmology, which is a promising idea that might allow one to make contact with phenomenology.

\subsection{Deformed Special Relativity}

Deformed special relativity (DSR) is concerned with the departure from Lorentz symmetry that results from the postulate that the Planck energy transforms like a (component of a) momentum four vector and remains an invariant, maximal energy scale. While the modified commutation relations necessarily give rise to some version of DSR, one can also try to extract information from the deformation of the Lorentz symmetry, or the addition law, directly. This gives rise to what Amelino-Camelia has dubbed 'test theories' [18]: simplified and reduced versions of the quantum theory with a minimal length. Working with these test theories has the advantage that one can make contact with phenomenology without working out the - still not very well understood - second quantization and interaction. It has the disadvantage that it makes the ambiguity in identifying physically-meaningful observables worse.

The literature on the topic is vast, and we can not cover it in totality here. For more details on the DSR phenomenology, the reader is referred to $[19,20]$. We will just mention the most relevant properties of these types of models here.

As we have seen earlier, a non-linear relation $\mathbf{p}(\mathbf{k})$, where $\mathbf{k}$ transforms under a normal Lorentz transformation, generates the deformed Lorentz transformation for $\mathbf{p}$ by Eq. (181). Note that in DSR it is the non-linearly transforming $\mathbf{p}$ that is considered the physical momentum, while the $\mathbf{k}$ that transforms under the normal Lorentz transformation is considered the pseudo-momentum. There is an infinite number of such functions, and thus there is an infinite number of ways to deform special relativity.

We have already met the common choices in the literature; they constitute bases in $\kappa$-Minkowski phase space, for example, the coordinates (211) proposed by Magueijo and Smolin in [211]. With this relation, a boost in the $z$-direction takes the form

$$
\begin{aligned}
\mathcal{P}_{0}^{\prime} & =\frac{\gamma\left(\mathcal{P}_{0}-v \mathcal{P}_{z}\right)}{1+(\gamma-1) \mathcal{P}_{0} / m_{\mathrm{Pl}}-\gamma v \mathcal{P}_{z} / m_{\mathrm{Pl}}} \\
\mathcal{P}_{z}^{\prime} & =\frac{\gamma\left(\mathcal{P}_{z}-v \mathcal{P}_{0}\right)}{1+(\gamma-1) \mathcal{P}_{0} / m_{\mathrm{Pl}}-\gamma v \mathcal{P}_{z} / m_{\mathrm{Pl}}} .
\end{aligned}
$$


This example, which has become known as DSR2, is particularly illustrative because this deformed Lorentz boost transforms $\left(m_{\mathrm{Pl}}, m_{\mathrm{Pl}}\right) \rightarrow\left(m_{\mathrm{Pl}}, m_{\mathrm{Pl}}\right)$, and thus keeps the Planck energy invariant. Note that since $k_{0} / k=\mathcal{P}_{0} / \mathcal{P}$, a so defined speed of light remains constant in this case.

Another example that has entered the literature under the name DSR1 [71] makes use of the bicrossproduct basis (209). Then, the dispersion relation for massless particles takes the form

$$
\cosh \left(\pi_{0} / m_{\mathrm{Pl}}\right)=\frac{1}{2} \frac{\vec{\pi}^{2}}{m_{\mathrm{Pl}}^{2}} e^{\pi_{0} / m_{\mathrm{Pl}}} .
$$

The relation between the momenta and the pseudo-momenta and their inverse has been worked out in [171]. In this example, the speed of massless particles that one derives from the dispersion relation depends on the energy of the particle. This effect may be observable in high-frequency light reaching Earth from distant sources, for example from $\gamma$-ray bursts. This interesting prediction is covered in more detail in $[26,19,20]$.

As discussed in Section 4.2.4, the addition law in this type of model has to be modified in order to obtain Lorentz-invariant conserved sums of momenta. This gives rise to the soccer-ball problem and can lead to changes in thresholds of particle interactions [19, 20, 78]. It had originally been argued that this would shift the GZK cut-off [17], but this argument has meanwhile been revised. However, in the 'test theory' one does not actually have a description of the particle interaction, so whether or not the kinematical considerations would be realized is unclear.

In these two DSR theories, it is usually assumed that the position variables conjugated to $\mathbf{k}$ are not commutative [99], thereby delivering a particular realization of $\kappa$-Minkowski space. In the DSR1 model, the speed of massless particles that one derives from the above dispersion relation (220) is energy dependent. However, the interpretation of that speed hinges on the meaning of the conjugated position-space coordinates, which is why it has also been argued that the physicallymeaningful speed is actually constant [301, 99]. Without an identification of observable positions, it is then also difficult to say whether this type of model actually realizes a minimal length. One can expect that recent work on the principle of relative locality [287, 286, 169, 27, 23, 21, 79] will shed light on this question. A forthcoming review [196] will be especially dedicated to the development of relative locality.

\subsection{Composite systems and statistical mechanics}

As mentioned previously, a satisfactory treatment of multi-particle states in those models in which the free particles' momenta are bound by a maximal energy scale is still lacking. Nevertheless, approaches to the description of composite systems or many particle states have been made, based on the modified commutation relations either without subscribing to the deformed Lorentz transformations, and thereby generically breaking Lorentz invariance, or by employing an ad hoc solution by rescaling the bound on the energy with the number of constituents. While these approaches are promising in so far that modified statistical mechanics at Planckian energies would allow one to use the early universe as a laboratory, they should be regarded with some caution, because the connection to the single particle description with deformed Lorentz symmetry is missing, and the case in which Lorentz symmetry is broken is strongly constrained already [191].

That having been said, the statistical mechanics from the $\kappa$-Poincaré algebra was investigated in general in [194, 109]. In [268] corrections to the effective Hamiltonian of macroscopic bodies have been studied, and in [264] observational consequences of modified commutation relations for a massive oscillator have been considered. In [172] statistical mechanics with a generalized uncertainty and possible applications for cosmology have been looked at. The partition functions of minimal-length quantized statistical mechanics have been derived in [257], in [6] the consequences of the GUP on the Liouville theorem were investigated, and in [84] the modification of the density of states and the arising consequences for black-hole thermodynamics were studied. In [242], one finds 
the effects of the GUP on the thermodynamics of ultra-relativistic particles in the early universe, and relativistic thermodynamics in [94]. [312] studied the equation of state for ultra-relativistic Fermi gases in compact stars, the ideal gas was addressed in [82] and photon gas thermodynamics in [320].

\subsection{Path-integral duality}

In Sections 3.2.4 and 3.4 we have discussed two motivations for limits of spacetime distances that manifest themselves in the Green's function. While one may question how convincing these motivations are, the idea is interesting and may be considered as a model on its own right. Such a modification that realizes a finite 'zero point length' of spacetime intervals had been suggested by Padmanabhan $[247,249,250,251]$ as a way to effectively take into account metric fluctuations below the Planck scale (the motivation from string theory discussed in Section 3.2.4 was added after the original proposal). This model has the merit of not requiring a modification of Lorentz invariance.

The starting point is to rewrite the Feynman propagator $G_{F}(x, y)$ as a sum over all paths $\gamma$ connecting $x$ and $y$

$$
G_{F}(x, y)=\sum_{\gamma} e^{-m D_{\gamma}(x, y)}=\int d \tau e^{-m \tau} K(x, y, \tau),
$$

where $D_{\gamma}(x, y)$ is the proper length of $\gamma$, and $m$ is a constant of dimension mass. Note that the length of the path depends on the background metric, which is why one expects it to be subject to quantum gravitational fluctuations. The path integral kernel is

$$
K(x, y, \tau)=\int \mathcal{D} x \exp \left(-\frac{m}{4} \int_{0}^{\tau} \mathrm{d} \tau^{\prime} g_{\mu \nu} \dot{x}^{\mu} \dot{x}^{\nu}\right),
$$

where a dot indicates a derivative with respect to $\tau^{\prime}$. The relevant difference between the middle and right expressions in (221) is that $D(x, y)$ has a square root in it. The equivalence has been shown using a Euclidean lattice approach in [251]. Once the propagator is brought into that form, one can apply Padmanabhan's postulated "principle of duality" according to which the weight for each path should be invariant under the transformation $D_{\gamma}(x, y) \rightarrow l_{\mathrm{Pl}}^{2} / D_{\gamma}(x, y)$. This changes the propagator (221) to

$$
\widetilde{G}_{F}(x, y)=\sum_{\gamma} \exp \left[-m\left(D_{\gamma}(x, y)+\frac{l_{\mathrm{Pl}}^{2}}{D_{\gamma}(x, y)}\right)\right] .
$$

Interestingly enough, it can be shown [251] that with this modification in the Schwinger representation, the path integral kernel remains unmodified, and one merely obtains a change of the weight

$$
\widetilde{G}_{F}(x, y)=\int \mathrm{d} \tau \exp \left[-m\left(\tau+\frac{l_{\mathrm{Pl}}^{2}}{\tau}\right)\right] K(x, y, \tau) .
$$

When one makes the Fourier transformation of this expression, the propagator in momentum space takes the form

$$
\widetilde{G}_{F}(p)=\frac{2 l_{\mathrm{Pl}}}{\sqrt{p^{2}+m^{2}}} K_{1}\left(2 l_{\mathrm{PI}} \sqrt{p^{2}+m^{2}}\right)
$$


where $K_{1}$ is the modified Bessel function of the first kind. This expression has the limiting values (compare to Eq. (109))

$$
\widetilde{G}_{F}(p) \rightarrow\left\{\begin{array}{ll}
\frac{1}{p^{2}+m^{2}} & \text { for } \sqrt{p^{2}+m^{2}} \ll m_{\mathrm{Pl}} \\
\frac{\exp \left(-2 l_{\mathrm{Pl}} \sqrt{p^{2}+m^{2}}\right)}{\sqrt{2 l_{\mathrm{Pl}}\left(p^{2}+m^{2}\right)^{3 / 4}}} & \text { for } m_{\mathrm{Pl}} \ll \sqrt{p^{2}+m^{2}}
\end{array} .\right.
$$

This postulated duality of the path integral thus suppresses the super-Planckian contributions to amplitudes. As mentioned in Section 3.2.4, in position space, the Feynman propagator differs from the ordinary one by the shift $(x-y)^{2} \rightarrow(x-y)^{2}+2 l_{\mathrm{Pl}}$. (This idea is so different not from that of March [219], who in 1936 proposed to replace ordinary spacetime distances with a modified distance $\mathrm{d} \tilde{s}=d s-\rho$. Though at that time, the 'minimal length' $\rho$ was supposed to be of about the size of the atomic nucleus. March's interpretation was that when the newly defined distance between two points vanishes, the points become indistinguishable.)

Some applications for this model for QED, for example the Casimir effect, have been worked out in [294, 282], and consequences for inflation and cosmological models have been looked at in $[295,192]$. For a recent criticism see [76].

\subsection{Direct applications of the uncertainty principle}

Maybe the most direct way to look for effects of the minimal length is to start from the GUP itself. This procedure is limited in its applicability because there are only so many insights one can gain from an inequality for variances of operators. However, cases that can be studied this way are everything that can be concluded from modifications of the Bekenstein argument, and with it corrections to the black-hole entropy that one obtains taking into account the modification of the uncertainty principle and modified dispersion relations.

Most interestingly, in [225, 28] it has been argued that comparing the corrections to the blackhole entropy obtained from the GUP to the corrections obtained in string theory and LQG may be used to restrict the functional form of the GUP.

It has also been argued that taking into account the GUP may give rise to black-hole remnants [2], a possibility that has been explored in many follow-up works, e.g., [87, 243, 315]. Corrections to the thermodynamical properties of a Schwarzschild black hole have been looked at in [22, 321, 100, 214, 217], the Reissner-Nordström black hole has been considered in [319], and black holes in anti-de Sitter space in [280, 281, 61]. Black-hole thermodynamics with a GUP has been studied in [201, 237, 54, 186], the thermodynamics of Kerr-Newman black holes in [315], and the entropy of a charged black hole in $f(R)$ gravity in [273]. In [80] the consequences of the GUP for self-dual black holes found in the mini-superspace approximation of LQC have been analyzed.

The thermodynamics of anti-de Sitter space has been looked at in [309], and the dynamics of the Taub cosmological model with GUP in [53]. The thermodynamics of Friedmann-RobertsonWalker in four-dimensional spacetimes with GUP can be found in [52, 216], and with additional dimensions in [279]. The relations of the GUP to holography in extra dimensions have been considered in [276], the effects of GUP on perfect fluids in cosmology in [215], and the entropy of the bulk scalar field in the Randall-Sundrum model with GUP in [185]. In [119] it has been suggested that there is a relationship between black-hole entropy and the cosmological constant. The relations of the GUP to Verlinde's entropic gravity have been discussed in [245]

\subsection{Miscellaneous}

While most of the work on modified uncertainty relations has focused on the GUP, the consequences of the spacetime uncertainty that arises in string theory for the spectrum of cosmological 
perturbations have been studied in [68]. In [269] it has been proposed that it might be possible to test Planck scale modifications of the energy-time uncertainty relation by monitoring tritium decay. It should also be mentioned that the classical mechanics of $\kappa$-Poincaré has been worked out in [206], and the kinematics of a classical free relativistic particle with deformed phase space in $[132,123,32]$. The effects of such a deformed phase space on scalar field cosmology have been investigated in [263].

In [179] an interesting consequence of the minimal length was studied, the implication of a finite bandwidth for physical fields. Making this connection allows one to then use theorems from classical information theory, such as Shannon's sampling theorem. It was shown in [179] that fields on a space with minimum length uncertainty can be reconstructed everywhere if known only on a discrete set of points (any set of points), if these points are, on average, spaced densely enough. These continuous fields then have a literally finite information density. In [181], it was shown that this information-theoretic meaning of the minimal length generalizes naturally to curved spacetime and in [182] it was then argued that for this reason spacetime would be simultaneously continuous and discrete in the same way that information can be.

A model for spacetime foam in terms of non-local interactions as a description for quantum gravitational effects, which serves as an origin of a minimal length scale, has been put forward in $[121,122]$. This model is interesting because it ties together three avenues towards a phenomenology of quantum gravity: the minimal length scale, decoherence from spacetime foam, and non-locality.

Finally, we mention that a minimum time and length uncertainty in rainbow gravity has been found in $[115,116,117]$. 


\section{Discussion}

After the explicit examples in Sections 3 and 4, here we will collect some general considerations.

One noteworthy remark for models with a minimal length scale is that discreteness seems neither necessary nor sufficient for the existence of a minimal length scale. String theory is an example that documents that discreteness is not necessary for a limit to the resolution of structures, and [60] offered example in which discreteness does not put a finite limit on the resolution of spatial distances (though the physical interpretation, or the observability of these quantities requires more study).

We have also seen that the minimal length scale is not necessarily the Planck length. In string theory, it is naturally the string scale that comes into play, or a product of the string coupling and the string scale if one takes into account D-branes. Also in ASG, or emergent gravity scenarios, the Planck mass might just appear as a coupling constant in some effective limit, while fundamentally some other constant is relevant. We usually talk about the Planck mass because we know of no higher energy scale that is relevant to the physics we know, so it is the obvious candidate, but not necessarily the right one.

\section{$5.1 \quad$ Interrelations}

The previously-discussed theories and models are related in various ways. We had already mentioned that the path-integral duality (Section 4.7) is possibly related to T-duality (Section 3.2.4) or conformal fluctuations in quantum gravity (Section 3.4), and that string theory is one of the reasons to study non-commutative geometries. In addition to this, it has also been argued that the coherent-state approach to non-commutative geometries represents another model for minimal length modified quantum mechanics [293]. The physics of black holes in light of the coherent state approach has been reviewed in [239].

DSR has been motivated by LQG, though no rigorous derivation exists to date. However, there are non-rigorous arguments that DSR may emerge from a semiclassical limit of quantum gravity theories in the form of an effective field theory with an energy dependent metric [29], or that DSR (in form of a $\kappa$-Poincaré algebra) may result from a version of path integral quantization [198]. In addition, it has been shown that in $2+1$ dimensional gravity coupled to matter, the gravitational degrees of freedom can be integrated out, leaving an effective field theory for the matter, which is a quantum field theory on $\kappa$-Minkowski spacetime, realizing a particular version of DSR [114]. Recently, it has also been suggested that DSR could arise via LQC [59].

As already mentioned, it has been argued in [74] that ASG may give rise to DSR if one carefully identifies the momentum and the pseudo-momentum. In [133] how the running of the Planck's mass can give rise to a modified dispersion relation was studied.

\subsection{Observable consequences}

The most relevant aspect of any model is to make contact with phenomenology. We have mentioned a few phenomenological consequences that are currently under study, but for completeness we summarize them here.

To begin with, experimental evidence that speaks for any one of the approaches to quantum gravity discussed in Section 3 will also shed light on the nature of a fundamental length scale. Currently, the most promising areas to look for such evidence are cosmology (in particular the polarization of the cosmic microwave background) and miscellaneous signatures of Lorentz-invariance violation. The general experimental possibilities to make headway on a theory of quantum gravity have been reviewed in $[19,164]$. One notable recent development, which is especially interesting for the question of a minimal length scale, is the possibility that direct evidence for the discrete nature of spacetime may be found in the emission spectra of primordial black holes, if such black holes exist and can be observed [50]. 
Signatures directly related to the minimal length proposal are a transplanckian cut-off that would make itself noticeable in the cosmic microwave background in the way that the spectrum of fluctuations would not be exactly scale invariant [220,68]. Imprints from scalar and tensor perturbations have been studied in $[37,38,36]$, and in $[88,89]$ it has been argued in that observable consequences arise at the level of the CMB bispectrum. Deformations of special relativity can lead to an energy-dependent dispersion, which might be an observable effect for photons reaching Earth from $\gamma$-ray bursts at high redshift $[26,19,20]$. Minimal length deformations do, in principle, give rise to computable correction terms to a large number of quantum mechanical phenomena (see Section 4.3.2). This allows one to put bounds on the parameters of the model. These bounds are presently many orders of magnitude away from the regime where one would naturally expect quantum gravitational effects. While it is therefore unlikely that evidence for a minimal length can be found in these experiments, it should be kept in mind that we do not strictly speaking know that the minimal length scale is identical to the Planck scale and not lower, and scientific care demands that every new range of parameter space be scrutinized.

Recently, it was proposed that a massive quantum mechanical oscillator might allow one to test Planck-scale physics [264] in a parameter range close to the Planck scale. This proposal should be regarded with caution because the deformations for composite systems used therein do not actually follow from the ones that were motivated by our considerations in Section 3, because the massive oscillator represents a multi-particle state. If one takes into account the ad-hoc solutions to the soccer-ball problem, that are necessary for consistency of the theory when considering multiparticle states (see Section 4.2.4), then the expected effect is suppressed by a mass many orders of magnitude above the Planck mass. Thus, it is unlikely that the proposed experiment will be sensitive to Planck-scale physics.

It is clearly desireable to be able to study composite systems and ensembles, which would allow us to make use of recent advances in quantum optics and data from the early high-density era of the universe. Thus, solving the soccer-ball problem is of central relevance for making contact between these models and phenomenology.

\title{
5.3 Is it possible that there is no minimal length?
}

\author{
"The last function of reason is to recognize that there are an infinity of things which \\ surpass it." \\ - Blaise Pascal
}

After having summarized all the motivations for the existence of a minimal length scale, we have to take care that our desire for harmony does not have us neglecting evidence to the contrary.

We have already discussed that there are various possibilities for a minimal length scale to make itself noticeable, and this does not necessarily mean that it appears as a lower bound on the spatial resolution. We could instead merely have a bound on products of spatial and temporal extensions. So in this sense there might not be a minimal length, just a minimal length scale. Therefore, we answer the question posed in this section's title in the affirmative. Let us then ask if it is possible that there is no minimal length scale.

The case for a minimal length scale seems clear in string theory and LQG, but it is less clear in emergent gravity scenarios. If gravity is emergent, and the Planck mass appears merely as a coupling constant in the effective limit, this raises the question, of there is some way in which the fundamental theory cannot have a limiting value at all.

In ASG, the arguments we have reviewed in Section 3.5 are suggestive but not entirely conclusive. The supporting evidence that we discussed comes from graviton scattering, and from a 
study of a particular type of Euclidean quantum spacetimes. ${ }^{13}$ Notwithstanding the question of whether general relativity actually has a (physically-meaningful) fixed point, the evidence for a minimal length is counterintuitive even in ASG, because gravity becomes weaker at high energies, so, naively, one would expect its distorting effects to also become weaker.

As Mead carefully pointed out in his article investigating the Heisenberg microscope with gravity:

"We have also neglected the effect of quantum fluctuations in the gravitational field. However, these would be expected to provide an additional source of uncertainty, not remove those already present. Hence, inclusion of this effect would, if anything, strengthen the result." ([222], p. B855)

That is correct, one might add, unless gravity itself weakens and counteracts the effect of the quantum fluctuations. In fact, in [51] the validity of the Hoop conjecture in a thought experiment testing short-distance structures has been re-examined in the context of ASG. It was found that the running of the Planck mass avoids the necessity of forming a trapped surface at the scale of the experiment. However, it was also found that still no information about the local physics can be transmitted to an observer in the asymptotic distance.

As previously mentioned, there is also no obvious reason for the existence of a minimal length scale in discrete approaches where the lattice spacing is taken to zero [13]. To study the question, one needs to investigate the behavior of suitably constructed observables in this limit. We also note the central role of the Hoop conjecture for our arguments, and that it is, for general configurations, an unproven conjecture.

These questions are presently very much under discussion; we mention them to show that the case is not as settled as it might have seemed from Sections 3 and 4.

13 Though it has meanwhile been shown that the fixed point behavior can be found also in the Lorentzian case [218]. 


\section{Summary}

We have seen in this review that there are many motivations for the existence of a minimal length scale. Various thought experiments suggest there are limits to how well we can resolve structures. String theory and LQG, presently the two most widely pursued approaches to quantum gravity, both bring with them a minimal length scale, if in very different realizations. It has been argued that a minimal length scale also exists in the scenario of ASG, and that non-commutative geometries have a minimal length scale built in already.

With that extensive motivation, many models have been proposed that aim at incorporating a minimal length scale into the quantum field theories of the standard model, rather than waiting for a theory of quantum gravity to be developed and eventually connected to the standard model. We have discussed some of these approaches, and also identified some key open problems. While a lot of work has been done directly studying the implications of modified dispersion relations, deformations of special relativity and a GUP, the underlying framework is not yet entirely understood. Most importantly, there is the question of how to construct physically-meaningful observables. One possibility to address this and some other open questions is to develop an axiomatic approach based on the geometry of phase space.

Exploring the consequences of a minimal length scale is one of the best motivated avenues to make contact with the phenomenology of quantum gravity, and to gain insights about the fundamental structure of space and time. 


\section{Acknowledgements}

I thank Xavier Calmet, Florian Girelli, Luis Garay, Steven Giddings, Arun Gupta, Amit Hagar, Achim Kempf, Jerzy Kowalski-Glikman, Boris Panes, Roberto Percacci, Stefan Scherer, Lee Smolin, Giorgio Torrieri and Phil Warnell for helpful feedback, and Renate Loll for suggesting this review - it was only by working on it that I realized an up-to-date comprehensive summary has been overdue. I am also very grateful for Luis Garay's 1994 review [120], which was an invaluable starting point.

I want to apologize to all those whose work was not treated in the depth it deserved. I have tried to make up for brevity with an extensive literature list. I hope the reader finds this compilation of work on the minimal length in quantum gravity scenarios useful. 


\section{Index}

$\kappa$-Minkowski, 54, 62, 66

$\kappa$-Poincaré, 9, 54

ASG, see Asymptotically Safe Gravity

Ashtekar variables, 32

Asymptotic darkness, 25

Asymptotically Safe Gravity, 37, 46, 68

Bicrossproduct, 54, 56, 62

Black hole, 9, 18, 21, 64

higher dimensional, 27

remnant, 64

Causal sets, 44

Classicalization, 45, 47

Clock synchronization, 17

Commutation relations, 8, 41, 47

Conformal invariance, 26

Cut-off, 8, 23

De Sitter space, 53

Deformed Special Relativity, 46, 61, 66

Dirichlet-branes, 27

Discrete spacetime, 44, 68

DSR, see Deformed Special Relativity

Emergent gravity, 38, 44, 67

Finite information density, 65

Generalized uncertainty principle, 13, 23, 47, 64 to all orders, 51

GUP, see Generalized Uncertainty Principle

Hawking-radiation, 9, 64

Hoop conjecture, 13, 21, 68

Hopf algebra, 9, 54

Jacobi identities, 51, 55

Locality bound, 45

Loop Quantum Cosmology, 32, 66

Loop Quantum Gravity, 32, 66

Lorentz invariance, 41, 46

Lorentz-invariance, 6

LQC, see Loop Quantum Cosmology

LQG, see Loop Quantum Gravity

Maximal localization states, 59
Modified dispersion relation, 47, 66

Momentum space curvature, 53

Non-commutative geometry, 8, 41, 54, 66

Non-commutative soliton, 44

Non-locality, 58, 61, 65

Observer independence, 46

Path-integral duality, 46, 63

Phase space, 48, 53

Planck scale, 6, 22, 47 energy dependent, 38 lowered, 10, 23

Planck's constant, 6, 48

Poisson tensor, 41

Principle of relative locality, 48, 58, 62

Pseudo-momentum, 48

Renormalizability, 7

Renormalization, 37

Renormalization group, 37

RG, see Renormalization group

Riemann surface, 22

Snyder basis, 53

Soccer-ball problem, 56

Spacetime uncertainty, 26, 65

Spectator problem, 56

Star product, 42

String scale, 22, 66

String Theory, 22

Symplectic form, 49

T-duality, 30

Thought experiment, 11

Time atoms, 6

Transplanckian problem, 9

Uncertainty principle, 11

Zero-point length, 32, 63 


\section{References}

[1] Abel, S. and Santiago, J., "Constraining the string scale: from Planck to weak and back again", J. Phys. G: Nucl. Part. Phys., 30, R83-R111 (2004). [DOI], [arXiv:hep-ph/0404237]. (Cited on page 23.)

[2] Adler, R.J., Chen, P. and Santiago, D.I., "The Generalized Uncertainty Principle and Black Hole Remnants", Gen. Relativ. Gravit., 33, 2101-2108 (2001). [DOI], [arXiv:gr-qc/0106080 [gr-qc]]. (Cited on page 64 .)

[3] Adler, R.J. and Santiago, D.I., "On gravity and the uncertainty principle", Mod. Phys. Lett. A, 14, 1371 (1999). [DOI], [arXiv:gr-qc/9904026]. (Cited on pages 10, 13, 15, and 16.)

[4] Agostini, A., Amelino-Camelia, G., Arzano, M., Marcianò, A. and Altair Tacchi, R., "Generalizing the Noether theorem for Hopf-algebra spacetime symmetries", Mod. Phys. Lett. A, 22, 1779-1786 (2007). [DOI], [ADS], [arXiv:hep-th/0607221 [hep-th]]. (Cited on page 60.)

[5] Ahluwalia-Khalilova, D.V., "Operational indistinguishability of doubly special relativities from special relativity", arXiv, e-print, (2002). [arXiv:gr-qc/0212128 [gr-qc]]. (Cited on page 58.)

[6] Ali, A.F., "Minimal Length in Quantum Gravity, Equivalence Principle and Holographic Entropy Bound", Class. Quantum Grav., 28, 065013 (2011). [DOI], [arXiv:1101.4181 [hep-th]]. (Cited on page 62.)

[7] Ali, A.F., Das, S. and Vagenas, E.C., "Discreteness of Space from the Generalized Uncertainty Principle", Phys. Lett. B, 678, 497-499 (2009). [DOI], [arXiv:0906.5396 [hep-th]]. (Cited on page 59.)

[8] Ali, A.F., Das, S. and Vagenas, E.C., "A proposal for testing quantum gravity in the lab", Phys. Rev. D, 84, 044013 (2011). [DOI], [arXiv:1107.3164 [hep-th]]. (Cited on page 59.)

[9] Amati, D., Ciafaloni, M. and Veneziano, G., "Superstring Collisions at Planckian Energies", Phys. Lett. B, 197, 81-88 (1987). [DOI]. (Cited on pages 10 and 25.)

[10] Amati, D., Ciafaloni, M. and Veneziano, G., "Classical and Quantum Gravity Effects from Planckian Energy Superstring Collisions", Int. J. Mod. Phys. A, 3, 1615-1661 (1988). [DOI]. (Cited on pages 10 and 25.)

[11] Amati, D., Ciafaloni, M. and Veneziano, G., "Higher order gravitational deflection and soft bremsstrahlung in Planckian energy superstring collisions", Nucl. Phys. B, 347, 550-580 (1990). [DOI]. (Cited on pages 10 and 25.)

[12] Amati, D., Ciafaloni, M. and Veneziano, G., "Towards an S-matrix description of gravitational collapse", J. High Energy Phys., 2008(02), 049 (2008). [DOI], [arXiv:0712.1209 [hep-th]]. (Cited on page 25.)

[13] Ambjørn, J., Jurkiewicz, J. and Loll, R., "Causal dynamical triangulations and the quest for quantum gravity", in Murugan, J., Weltman, A. and Ellis, G.F.R., eds., Foundations of Space and Time: Reflections on Quantum Gravity, pp. 321-337, (Cambridge University Press, Cambridge; New York, 2012). [arXiv:1004.0352 [hep-th]]. (Cited on page 68.)

[14] Amelino-Camelia, G., "Testable scenario for relativity with minimum length", Phys. Lett. B, 510 255-263 (2001). [DOI], [arXiv:hep-th/0012238 [hep-th]]. (Cited on page 46.)

[15] Amelino-Camelia, G., "Doubly special relativity", Nature, 418, 34-35 (2002). [DOI], [arXiv:grqc/0207049 [gr-qc]]. (Cited on page 46.)

[16] Amelino-Camelia, G., "Doubly-special relativity: First results and key open problems", Int. J. Mod. Phys. D, 11, 1643-1669 (2002). [DOI], [arXiv:gr-qc/0210063 [gr-qc]]. (Cited on page 58.) 
[17] Amelino-Camelia, G., "Kinematical solution of the UHE-cosmic-ray puzzle without a preferred class of inertial observers", Int. J. Mod. Phys. D, 12, 1211-1226 (2003). [DOI], [arXiv:astro-ph/0209232 [astro-ph]]. (Cited on page 62.)

[18] Amelino-Camelia, G., "Planck-scale Lorentz-symmetry test theories", arXiv, e-print, (2004). [arXiv:astro-ph/0410076 [astro-ph]]. (Cited on page 61.)

[19] Amelino-Camelia, G., "Quantum Gravity Phenomenology", arXiv, e-print, (2008). [arXiv:0806.0339 [gr-qc]]. (Cited on pages 61, 62, 66, and 67.)

[20] Amelino-Camelia, G., "Doubly-Special Relativity: Facts, Myths and Some Key Open Issues", Symmetry, 2, 230-271 (2010). [DOI], [arXiv:1003.3942 [gr-qc]]. (Cited on pages 61, 62, and 67.)

[21] Amelino-Camelia, G., "On the fate of Lorentz symmetry in relative-locality momentum spaces", Phys. Rev. D, 85, 084034 (2012). [DOI], [arXiv:1110.5081 [hep-th]]. (Cited on pages 48, 57, 58, and 62.)

[22] Amelino-Camelia, G., Arzano, M., Ling, Y. and Mandanici, G., "Black-hole thermodynamics with modified dispersion relations and generalized uncertainty principles", Class. Quantum Grav., 23, 2585-2606 (2006). [DOI], [arXiv:gr-qc/0506110 [gr-qc]]. (Cited on page 64.)

[23] Amelino-Camelia, G., Freidel, L., Kowalski-Glikman, J. and Smolin, L., "The principle of relative locality", Phys. Rev. D, 84, 084010 (2011). [DOI], [arXiv:1101.0931 [hep-th]]. (Cited on pages 48, 57,58 , and 62.)

[24] Amelino-Camelia, G., Freidel, L., Kowalski-Glikman, J. and Smolin, L., "Relative locality and the soccer ball problem", Phys. Rev. D, 84, 087702 (2011). [DOI], [arXiv:1104.2019 [hep-th]]. (Cited on page 56.)

[25] Amelino-Camelia, G., Lukierski, J. and Nowicki, A., "Distance measurement and $\kappa$-deformed propagation of light and heavy probes", Int. J. Mod. Phys. A, 14, 4575-4588 (1999). [DOI], [arXiv:grqc/9903066 [gr-qc]]. (Cited on page 48.)

[26] Amelino-Camelia, G. and Majid, S., "Waves on noncommutative space-time and gamma-ray bursts", Int. J. Mod. Phys. A, 15, 4301-4324 (2000). [DOI], [arXiv:hep-th/9907110 [hep-th]]. (Cited on pages $48,58,62$, and 67.$)$

[27] Amelino-Camelia, G., Matassa, M., Mercati, F. and Rosati, G., "Taming Nonlocality in Theories with Planck-Scale Deformed Lorentz Symmetry", Phys. Rev. Lett., 106, 071301 (2011). [DOI], [arXiv:1006.2126 [gr-qc]]. (Cited on pages 48, 57, 58, and 62.)

[28] Amelino-Camelia, G., Procaccini, A. and Arzano, M., "A glimpse at the flat-spacetime limit of quantum gravity using the Bekenstein argument in reverse", Int. J. Mod. Phys. D, 13, 2337-2343 (2004). [DOI], [arXiv:hep-th/0506182 [hep-th]]. (Cited on page 64.)

[29] Amelino-Camelia, G., Smolin, L. and Starodubtsev, A., "Quantum symmetry, the cosmological constant and Planck scale phenomenology", Class. Quantum Grav., 21, 3095-3110 (2004). [DOI], [arXiv:hep-th/0306134 [hep-th]]. (Cited on page 66.)

[30] Anber, M.M. and Donoghue, J.F., "On the running of the gravitational constant", Phys. Rev. D, 85, 104016 (2012). [DOI], [arXiv:1111.2875 [hep-th]]. (Cited on page 41.)

[31] Arzano, M., "Anatomy of a deformed symmetry: Field quantization on curved momentum space", Phys. Rev. D, 83, 025025 (2011). [DOI], [arXiv:1009.1097 [hep-th]]. (Cited on page 60.)

[32] Arzano, M. and Kowalski-Glikman, J., "Kinematics of a relativistic particle with de Sitter momentum space", Class. Quantum Grav., 28, 105009 (2011). [DOI], [arXiv:1008.2962 [hep-th]]. (Cited on page 65.) 
[33] Arzano, M., Kowalski-Glikman, J. and Walkus, A., "Lorentz invariant field theory on $\kappa$-Minkowski space", Class. Quantum Grav., 27, 025012 (2010). [DOI], [arXiv:0908.1974 [hep-th]]. (Cited on page 60.)

[34] Arzano, M. and Marciano, A., "Fock space, quantum fields and $\kappa$-Poincaré symmetries", Phys. Rev. D, 76, 125005 (2007). [DOI], [arXiv:0707.1329 [hep-th]]. (Cited on page 60.)

[35] Arzano, M. and Marcianò, A., "Symplectic geometry and Noether charges for Hopf algebra spacetime symmetries", Phys. Rev. D, 75, 081701 (2007). [DOI], [arXiv:hep-th/0701268 [hep-th]]. (Cited on page 60. .)

[36] Ashoorioon, A., Hovdebo, J.L. and Mann, R.B., "Running of the spectral index and violation of the consistency relation between tensor and scalar spectra from trans-Planckian physics", Nucl. Phys. $B, \mathbf{7 2 7}, 63-76$ (2005). [DOI], [arXiv:gr-qc/0504135 [gr-qc]]. (Cited on page 67.)

[37] Ashoorioon, A., Kempf, A. and Mann, R.B., "Minimum length cutoff in inflation and uniqueness of the action", Phys. Rev. D, 71, 023503 (2005). [DOI], [arXiv:astro-ph/0410139 [astro-ph]]. (Cited on page 67.)

[38] Ashoorioon, A. and Mann, R.B., "On the tensor/scalar ratio in inflation with UV cut off", Nucl. Phys. B, 716, 261-279 (2005). [DOI], [arXiv:gr-qc/0411056 [gr-qc]]. (Cited on page 67.)

[39] Ashtekar, A., "New Variables for Classical and Quantum Gravity", Phys. Rev. Lett., 57, 2244-2247 (1986). [DOI]. (Cited on page 32.)

[40] Ashtekar, A., Bojowald, M. and Lewandowski, J., "Mathematical structure of loop quantum cosmology", Adv. Theor. Math. Phys., 7, 233-268 (2003). [arXiv:gr-qc/0304074]. (Cited on pages 34 and 35.)

[41] Ashtekar, A. and Lewandowski, J., "Quantum theory of geometry. II: Volume operators", Adv. Theor. Math. Phys., 1, 388-429 (1998). [arXiv:gr-qc/9711031]. (Cited on page 34.)

[42] Ashtekar, A. and Lewandowski, J., "Background independent quantum gravity: a status report", Class. Quantum Grav., 21, R53-R152 (2004). [DOI], [arXiv:gr-qc/0404018]. (Cited on page 32.)

[43] Ashtekar, A., Pawlowski, T., Singh, P. and Vandersloot, K., "Loop quantum cosmology of $k=1$ FRW models", Phys. Rev. D, 75, 024035 (2007). [DOI], [arXiv:gr-qc/0612104]. (Cited on page 36.)

[44] Ashtekar, A. and Singh, P., "Loop Quantum Cosmology: A Status Report", Class. Quantum Grav., 28, 213001 (2011). [DOI], [arXiv:1108.0893 [gr-qc]]. (Cited on pages 34 and 36.)

[45] Bachas, C.P., "Lectures on D-branes", arXiv, e-print, (1998). [arXiv:hep-th/9806199]. (Cited on pages 27 and 30.)

[46] Bachmann, S. and Kempf, A., "The Transplanckian Question and the Casimir Effect", arXiv, e-print, (2005). [arXiv:gr-qc/0504076 [gr-qc]]. (Cited on page 59.)

[47] Banks, T., "A critique of pure string theory: Heterodox opinions of diverse dimensions", arXiv, e-print, (2003). [arXiv:hep-th/0306074]. (Cited on page 25.)

[48] Banks, T. and Fischler, W., "A Model for High Energy Scattering in Quantum Gravity", arXiv, e-print, (1999). [arXiv:hep-th/9906038]. (Cited on page 25.)

[49] Barceló, C., Liberati, S. and Visser, M., "Analogue Gravity", Living Rev. Relativity, 14, lrr-2011-03 (2011). [arXiv:gr-qc/0505065 [gr-qc]]. URL (accessed 20 January 2012): http://www.livingreviews.org/lrr-2011-3. (Cited on page 44.)

[50] Barrau, A., Cailleteau, T., Cao, X., Diaz-Polo, J. and Grain, J., "Probing Loop Quantum Gravity with Evaporating Black Holes", Phys. Rev. Lett., 107, 251301 (2011). [DOI], [arXiv:1109.4239 [grqc]]. (Cited on page 66.) 
[51] Basu, S. and Mattingly, D., "Asymptotic Safety, Asymptotic Darkness, and the hoop conjecture in the extreme UV", Phys. Rev. D, 82, 124017 (2010). [DOI], [arXiv:1006.0718 [hep-th]]. (Cited on pages 39 and 68.)

[52] Battisti, M.V. and Montani, G., "The Big-Bang singularity in the framework of a Generalized Uncertainty Principle", Phys. Lett. B, 656, 96-101 (2007). [DOI], [arXiv:gr-qc/0703025 [gr-qc]]. (Cited on page 64 .)

[53] Battisti, M.V. and Montani, G., "Quantum dynamics of the Taub universe in a generalized uncertainty principle framework", Phys. Rev. D, 77, 023518 (2008). [DOI], [arXiv:0707.2726 [gr-qc]]. (Cited on page 64.)

[54] Bina, A., Jalalzadeh, S. and Moslehi, A., "Quantum Black Hole in the Generalized Uncertainty Principle Framework", Phys. Rev. D, 81, 023528 (2010). [DOI], [arXiv:1001.0861 [gr-qc]]. (Cited on page 64.)

[55] Blaut, A., Daszkiewicz, M., Kowalski-Glikman, J. and Nowak, S., "Phase spaces of doubly special relativity", Phys. Lett. B, 582, 82-85 (2004). [DOI], [arXiv:hep-th/0312045 [hep-th]]. (Cited on page 53.)

[56] Bojowald, M., "Absence of singularity in loop quantum cosmology", Phys. Rev. Lett., 86, 5227-5230 (2001). [DOI], [arXiv:gr-qc/0102069]. (Cited on page 36.)

[57] Bojowald, M., "Dynamical coherent states and physical solutions of quantum cosmological bounces", Phys. Rev. D, 75, 123512 (2007). [DOI], [arXiv:gr-qc/0703144]. (Cited on pages 34 and 36.)

[58] Bojowald, M., "Loop Quantum Cosmology", Living Rev. Relativity, 11, lrr-2008-4 (2008). URL (accessed 20 January 2012):

http://www.livingreviews.org/lrr-2008-4. (Cited on pages 34 and 36.)

[59] Bojowald, M., "Quantum Geometry and Quantum Dynamics at the Planck Scale", in KowalskiGlikman, J., Durka, R. and Szczachor, M., eds., The Planck Scale, Proceedings of the XXV Max Born Symposium, Wroclaw, Poland, 29 June-03 July 2009, AIP Conference Proceedings, 1196, pp. 62-71, (American Institute of Physics, Melville, NY, 2009). [DOI], [arXiv:0910.2936 [gr-qc]]. (Cited on page 66.)

[60] Bojowald, M. and Kempf, A., "Generalized uncertainty principles and localization of a particle in discrete space", Phys. Rev. D, 86, 085017 (2012). [DOI], [arXiv:1112.0994 [hep-th]]. (Cited on pages 44 and 66.)

[61] Bolen, B. and Cavaglia, M., "(Anti-)de Sitter black hole thermodynamics and the generalized uncertainty principle", Gen. Relativ. Gravit., 37, 1255-1262 (2005). [DOI], [arXiv:gr-qc/0411086 [gr-qc]]. (Cited on page 64.)

[62] Bombelli, L., Henson, J. and Sorkin, R.D., "Discreteness without symmetry breaking: A Theorem", Mod. Phys. Lett. A, 24, 2579-2587 (2009). [DOI], [arXiv:gr-qc/0605006 [gr-qc]]. (Cited on page 44.)

[63] Bombelli, L. and Meyer, D.A., "The origin of Lorentzian geometry", Phys. Lett. A, 141, 226-228 (1989). [DOI]. (Cited on page 44.)

[64] Born, M., "A suggestion for unifying quantum theory and relativity", Proc. R. Soc. London, Ser. A, 165, 291-303 (1938). [DOI]. (Cited on page 48.)

[65] Bouaziz, D. and Bawin, M., "Regularization of the Singular Inverse Square Potential in Quantum Mechanics with a Minimal length", Phys. Rev. A, 76, 032112 (2007). [DOI], [arXiv:0711.0599 [quantph]]. (Cited on page 59.)

[66] Bouaziz, D. and Bawin, M., "Singular inverse square potential in arbitrary dimensions with a minimal length: Application to the motion of a dipole in a cosmic string background", Phys. Rev. A, $\mathbf{7 8}$, 032110 (2008). [DOI], [arXiv:1009.0930 [quant-ph]]. (Cited on page 59.) 
[67] Bouaziz, D. and Ferkous, N., "Hydrogen atom in momentum space with a minimal length", Phys. Rev. A, 82, 022105 (2010). [DOI], [arXiv:1009.0935 [quant-ph]]. (Cited on page 59.)

[68] Brandenberger, R.H. and Ho, P.-M., "Noncommutative space-time, stringy space-time uncertainty principle, and density fluctuations", Phys. Rev. D, 66, 023517 (2002). [DOI], [arXiv:hep-th/0203119 [hep-th]]. (Cited on pages 65 and 67.)

[69] Brau, F., "Minimal length uncertainty relation and hydrogen atom", J. Phys. A: Math. Gen., 32, 7691-7696 (1999). [DOI], [arXiv:quant-ph/9905033 [quant-ph]]. (Cited on page 59.)

[70] Bronstein, M., "Quantentheorie schwacher Gravitationsfelder", Phys. Z. Sowjetunion, 9, 140-157 (1936). (Cited on pages 6 and 7.)

[71] Bruno, N.R., Amelino-Camelia, G. and Kowalski-Glikman, J., "Deformed boost transformations that saturate at the Planck scale", Phys. Lett. B, 522, 133-138 (2001). [DOI], [arXiv:hep-th/0107039 [hep-th]]. (Cited on page 62.)

[72] Calmet, X., Graesser, M. and Hsu, S.D.H., "Minimum length from quantum mechanics and general relativity", Phys. Rev. Lett., 93, 211101 (2004). [DOI], [arXiv:hep-th/0405033]. (Cited on page 19.)

[73] Calmet, X., Graesser, M.L. and Hsu, S.D.H., "Minimum length from first principles", Int. J. Mod. Phys. D, 14, 2195-2200 (2005). [DOI], [arXiv:hep-th/0505144]. (Cited on page 19.)

[74] Calmet, X., Hossenfelder, S. and Percacci, R., "Deformed Special Relativity from Asymptotically Safe Gravity", Phys. Rev. D, 82, 124024 (2010). [DOI], [arXiv:1008.3345 [gr-qc]]. (Cited on pages 39, $41,47,57$, and 66.)

[75] Camacho, A., "Generalized uncertainty principle and quantum electrodynamics", Gen. Relativ. Gravit., 35, 1153-1160 (2003). [DOI], [arXiv:gr-qc/0303061 [gr-qc]]. (Cited on page 60.)

[76] Campo, D., "Problems with models of a fundamental length", arXiv, e-print, (2010). [arXiv:1004.5324 [gr-qc]]. (Cited on page 64.)

[77] Carmona, J.M., Cortes, J.L., Indurain, J. and Mazon, D., "Quantum Noncanonical Field Theory: Symmetries and Interaction", Phys. Rev. D, 80, 105014 (2009). [DOI], [arXiv:0905.1901 [hep-th]]. (Cited on page 60.)

[78] Carmona, J.M., Cortes, J.L. and Mazon, D., "Asymptotic approach to Special Relativity compatible with a relativistic principle", Phys. Rev. D, 82, 085012 (2010). [DOI], [arXiv:1007.3190 [gr-qc]]. (Cited on page 62.)

[79] Carmona, J.M., Cortes, J.L., Mazon, D. and Mercati, F., "About Locality and the Relativity Principle Beyond Special Relativity", Phys. Rev. D, 84, 085010 (2011). [DOI], [arXiv:1107.0939 [hep-th]]. (Cited on pages 58 and 62.)

[80] Carr, B., Modesto, L. and Prémont-Schwarz, I., "Generalized Uncertainty Principle and Self-dual Black Holes", arXiv, e-print, (2011). [arXiv:1107.0708 [gr-qc]]. (Cited on page 64.)

[81] Cavaglia, M. and Das, S., "How classical are TeV scale black holes?", Class. Quantum Grav., 21, 4511-4522 (2004). [DOI], [arXiv:hep-th/0404050 [hep-th]]. (Cited on page 61.)

[82] Chandra, N. and Chatterjee, S., "Thermodynamics of Ideal Gas in Doubly Special Relativity", Phys. Rev. D, 85, 045012 (2012). [DOI], [arXiv:1108.0896 [gr-qc]]. (Cited on page 63.)

[83] Chang, L.N., Lewis, Z., Minic, D. and Takeuchi, T., "On the Minimal Length Uncertainty Relation and the Foundations of String Theory", Adv. High Energy Phys., 2011, 493514 (2011). [DOI], [arXiv:1106.0068 [hep-th]]. (Cited on page 59.) 
[84] Chang, L.N., Minic, D., Okamura, N. and Takeuchi, T., "The Effect of the minimal length uncertainty relation on the density of states and the cosmological constant problem", Phys. Rev. D, 65, 125028 (2002). [DOI], [arXiv:hep-th/0201017 [hep-th]]. (Cited on page 62.)

[85] Chang, L.N., Minic, D., Okamura, N. and Takeuchi, T., "Exact solution of the harmonic oscillator in arbitrary dimensions with minimal length uncertainty relations", Phys. Rev. D, 65, 125027 (2002). [DOI], [arXiv:hep-th/0111181 [hep-th]]. (Cited on page 59.)

[86] Chang, L.N., Minic, D. and Takeuchi, T., "Quantum Gravity, Dynamical Energy-Momentum Space and Vacuum Energy", Mod. Phys. Lett. A, 25, 2947-2954 (2010). [DOI], [arXiv:1004.4220 [hep-th]]. (Cited on page 57.)

[87] Chen, P. and Adler, R.J., "Black hole remnants and dark matter", Nucl. Phys. B (Proc. Suppl.), 124, 103-106 (2003). [DOI], [arXiv:gr-qc/0205106 [gr-qc]]. (Cited on page 64.)

[88] Chialva, D., "Enhanced CMBR non-Gaussianities from Lorentz violation", J. Cosmol. Astropart. Phys., 2012(01), 037 (2012). [DOI], [arXiv:1106.0040 [hep-th]]. (Cited on page 67.)

[89] Chialva, D., "Signatures of very high energy physics in the squeezed limit of the bispectrum", $J$. Cosmol. Astropart. Phys., 2012(10), 037 (2012). [DOI], [arXiv:1108.4203 [astro-ph.CO]]. (Cited on page 67.)

[90] Ciafaloni, M. and Colferai, D., "Quantum Tunneling and Unitarity Features of an S-matrix for Gravitational Collapse", J. High Energy Phys., 2009(12), 062 (2009). [DOI], [arXiv:0909.4523 [hepth]]. (Cited on page 25.)

[91] Coleman, S.R., Preskill, J. and Wilczek, F., "Quantum hair on black holes", Nucl. Phys. B, 378, 175-246 (1992). [DOI], [arXiv:hep-th/9201059]. (Cited on page 18.)

[92] Cunliff, C., "Conformal fluctuations do not establish a minimum length", arXiv, e-print, (2012). [arXiv:1201.2247 [gr-qc]]. (Cited on page 37.)

[93] Dadic, I., Jonke, L. and Meljanac, S., "Harmonic oscillator with minimal length uncertainty relations and ladder operators", Phys. Rev. D, 67, 087701 (2003). [DOI], [arXiv:hep-th/0210264 [hep-th]]. (Cited on page 59.)

[94] Das, S., Ghosh, S. and Roychowdhury, D., "Relativistic Thermodynamics with an Invariant Energy Scale", Phys. Rev. D, 80, 125036 (2009). [DOI], [arXiv:0908.0413 [hep-th]]. (Cited on page 63.)

[95] Das, S. and Mann, R.B., "Planck scale effects on some low energy quantum phenomena", Phys. Lett. $B, \mathbf{7 0 4}, 596-599$ (2011). [DOI], [arXiv:1109.3258 [hep-th]]. (Cited on page 59.)

[96] Das, S. and Vagenas, E.C., "Universality of Quantum Gravity Corrections", Phys. Rev. Lett., 101, 221301 (2008). [DOI], [arXiv:0810.5333 [hep-th]]. (Cited on page 59.)

[97] Das, S. and Vagenas, E.C., "Phenomenological Implications of the Generalized Uncertainty Principle", Can. J. Phys., 87, 233-240 (2009). [DOI], [arXiv:0901.1768 [hep-th]]. (Cited on page 59.)

[98] Das, S., Vagenas, E.C. and Ali, A.F., "Discreteness of Space from GUP II: Relativistic Wave Equations", Phys. Lett. B, 690, 407-412 (2010). [DOI], [arXiv:1005.3368 [hep-th]]. (Cited on page 60.)

[99] Daszkiewicz, M., Imilkowska, K. and Kowalski-Glikman, J., "Velocity of particles in doubly special relativity", Phys. Lett. A, 323, 345-350 (2004). [DOI], [arXiv:hep-th/0304027 [hep-th]]. (Cited on pages 58 and 62.)

[100] Dehghani, M., "Corrected black hole's thermodynamics and tunneling radiation with generalized uncertainty principle and modified dispersion relation", Int. J. Theor. Phys., 50, 618-624 (2011). [DOI]. (Cited on page 64.) 
[101] Doplicher, S., Fredenhagen, K. and Roberts, J.E., "The quantum structure of space-time at the Planck scale and quantum fields", Commun. Math. Phys., 172, 187-220 (1995). [DOI], [arXiv:hepth/0303037]. (Cited on pages 21 and 22.)

[102] Dorsch, G. and Nogueira, J.A., "Maximally Localized States in Quantum Mechanics with a Modified Commutation Relation to All Orders", Int. J. Mod. Phys. A, 27, 1250113 (2012). [DOI], [arXiv:1106.2737 [hep-th]]. (Cited on page 59.)

[103] Douglas, M.R., Kabat, D.N., Pouliot, P. and Shenker, S.H., "D-branes and short distances in string theory", Nucl. Phys. B, 485, 85-127 (1997). [DOI], [arXiv:hep-th/9608024]. (Cited on pages 27 and 30.)

[104] Douglas, M.R. and Nekrasov, N.A., "Noncommutative field theory", Rev. Mod. Phys., 73, 977-1029 (2001). [DOI], [arXiv:hep-th/0106048 [hep-th]]. (Cited on pages 41 and 60.)

[105] Dvali, G., Folkerts, S. and Germani, C., "Physics of Trans-Planckian Gravity", Phys. Rev. D, 84, 024039 (2011). [DOI], [arXiv:1006.0984 [hep-th]]. (Cited on pages 45 and 47.)

[106] Dvali, G. and Gomez, C., "Self-Completeness of Einstein Gravity", arXiv, e-print, (2010). [arXiv:1005.3497 [hep-th]]. (Cited on pages 45 and 47.)

[107] Eardley, D.M. and Giddings, S.B., "Classical black hole production in high-energy collisions", Phys. Rev. D, 66, 044011 (2002). [DOI], [arXiv:gr-qc/0201034]. (Cited on page 13.)

[108] Falls, K., Litim, D.F. and Raghuraman, A., "Black holes and asymptotically safe gravity", Int. J. Mod. Phys. A, 27, 1250019 (2012). [DOI], [arXiv:1002.0260 [hep-th]]. (Cited on page 39.)

[109] Fityo, T.V., "Statistical physics in deformed spaces with minimal length", Phys. Lett. A, 372, 58725877 (2008). [DOI]. (Cited on page 62.)

[110] Flint, H.T., "Relativity and the quantum theory", Proc. R. Soc. London, Ser. A, 117, 630-637 (1928). [DOI]. (Cited on page 6.)

[111] Fontanini, M., Spallucci, E. and Padmanabhan, T., "Zero-point length from string fluctuations", Phys. Lett. B, 633, 627-630 (2006). [DOI], [arXiv:hep-th/0509090]. (Cited on page 31.)

[112] Frassino, A.M. and Panella, O., "The Casimir Effect in Minimal Length Theories Based on a Generalized Uncertainity Principle", Phys. Rev. D, 85, 045030 (2012). [DOI], [arXiv:1112.2924 [hep-th]]. (Cited on page 59.)

[113] Freidel, L., Kowalski-Glikman, J. and Nowak, S., "Field theory on $\kappa$-Minkowski space revisited: Noether charges and breaking of Lorentz symmetry", Int. J. Mod. Phys. A, 23, 2687-2718 (2008). [DOI], [arXiv:0706.3658 [hep-th]]. (Cited on page 60.)

[114] Freidel, L., Kowalski-Glikman, J. and Smolin, L., "2+1 gravity and doubly special relativity", Phys. Rev. D, 69, 044001 (2004). [DOI], [arXiv:hep-th/0307085 [hep-th]]. (Cited on page 66.)

[115] Galán, P. and Mena Marugán, G.A., "Quantum time uncertainty in a gravity's rainbow formalism", Phys. Rev. D, 70, 124003 (2004). [DOI], [arXiv:gr-qc/0411089 [gr-qc]]. (Cited on page 65.)

[116] Galán, P. and Mena Marugán, G.A., "Length uncertainty in a gravity's rainbow formalism", Phys. Rev. D, 72, 044019 (2005). [DOI], [arXiv:gr-qc/0507098 [gr-qc]]. (Cited on page 65.)

[117] Galán, P. and Mena Marugán, G.A., "Entropy and temperature of black holes in a gravity's rainbow", Phys. Rev. D, 74, 044035 (2006). [DOI], [arXiv:gr-qc/0608061 [gr-qc]]. (Cited on page 65.)

[118] Gambini, R. and Pullin, J., A First Course in Loop Quantum Gravity, (Oxford University Press, Oxford; New York, 2011). [Google Books]. (Cited on pages 32 and 34.) 
[119] Garattini, R., "Modified Dispersion Relations: from Black-Hole Entropy to the Cosmological Constant", Int. J. Mod. Phys.: Conf. Ser., 14, 326-336 (2012). [DOI], [arXiv:1112.1630 [gr-qc]]. (Cited on page 64.)

[120] Garay, L.J., "Quantum gravity and minimum length", Int. J. Mod. Phys. A, 10, 145-166 (1995). [DOI], [arXiv:gr-qc/9403008 [gr-qc]]. (Cited on pages 44 and 70.)

[121] Garay, L.J., "Spacetime Foam as a Quantum Thermal Bath", Phys. Rev. Lett., 80, 2508-2511 (1998). [DOI], [arXiv:gr-qc/9801024 [gr-qc]]. (Cited on page 65.)

[122] Garay, L.J., "Thermal properties of spacetime foam", Phys. Rev. D, 58, 124015 (1998). [DOI], [arXiv:gr-qc/9806047 [gr-qc]]. (Cited on page 65.)

[123] Ghosh, S., "A Lagrangian for DSR Particle and the Role of Noncommutativity", Phys. Rev. D, 74, 084019 (2006). [DOI], [arXiv:hep-th/0608206 [hep-th]]. (Cited on page 65.)

[124] Ghosh, S., "Generalized Uncertainty Principle and Klein Paradox", arXiv, e-print, (2012). [arXiv:1202.1962 [hep-th]]. (Cited on page 59.)

[125] Ghosh, S. and Roy, P., "Stringy' Coherent States Inspired By Generalized Uncertainty Principle", Phys. Lett. B, 711, 423-427 (2012). [DOI], [arXiv:1110.5136 [hep-th]]. (Cited on page 59.)

[126] Giddings, S.B., "Locality in quantum gravity and string theory", Phys. Rev. D, 74, 106006 (2006). [DOI], [arXiv:hep-th/0604072]. (Cited on page 44.)

[127] Giddings, S.B., Gross, D.J. and Maharana, A., "Gravitational effects in ultrahigh-energy string scattering", Phys. Rev. D, 77, 046001 (2008). [DOI], [arXiv:0705.1816 [hep-th]]. (Cited on page 25.)

[128] Giddings, S.B. and Lippert, M., "Precursors, black holes, and a locality bound", Phys. Rev. D, 65, 024006 (2002). [DOI], [arXiv:hep-th/0103231]. (Cited on page 44.)

[129] Giddings, S.B. and Lippert, M., "The information paradox and the locality bound", Phys. Rev. D, 69, 124019 (2004). [DOI], [arXiv:hep-th/0402073]. (Cited on page 44.)

[130] Giddings, S.B., Schmidt-Sommerfeld, M. and Andersen, J.R., "High energy scattering in gravity and supergravity", Phys. Rev. D, 82, 104022 (2010). [DOI], [arXiv:1005.5408 [hep-th]]. (Cited on page 25.)

[131] Giddings, S.B. and Thomas, S.D., "High energy colliders as black hole factories: The end of short distance physics", Phys. Rev. D, 65, 056010 (2002). [DOI], [arXiv:hep-ph/0106219]. (Cited on page 25.)

[132] Girelli, F., Konopka, T., Kowalski-Glikman, J. and Livine, E.R., "The free particle in deformed special relativity", Phys. Rev. D, 73, 045009 (2006). [DOI], [arXiv:hep-th/0512107 [hep-th]]. (Cited on pages 54,55 , and 65 .)

[133] Girelli, F., Liberati, S., Percacci, R. and Rahmede, C., "Modified Dispersion Relations from the Renormalization Group of Gravity", Class. Quantum Grav., 24, 3995-4008 (2007). [DOI], [arXiv:grqc/0607030 [gr-qc]]. (Cited on page 66.)

[134] Girelli, F. and Livine, E.R., "Physics of Deformed Special Relativity: Relativity Principle revisited", arXiv, e-print, (2004). [arXiv:gr-qc/0412004 [gr-qc]]. (Cited on page 56.)

[135] Girelli, F. and Livine, E.R., "Non-Commutativity of Effective Space-Time Coordinates and the Minimal Length", arXiv, e-print, (2007). [arXiv:0708.3813 [hep-th]]. (Cited on page 52.)

[136] Girelli, F. and Livine, E.R., "Special relativity as a non commutative geometry: Lessons for deformed special relativity", Phys. Rev. D, 81, 085041 (2010). [DOI], [arXiv:gr-qc/0407098 [gr-qc]]. (Cited on page 57.) 
[137] Gopakumar, R., Minwalla, S. and Strominger, A., "Noncommutative solitons", J. High Energy Phys., 2000(05), 020 (2000). [DOI], [arXiv:hep-th/0003160 [hep-th]]. (Cited on pages 43 and 44.)

[138] Gorelik, G.E., "Matvei Bronstein and quantum gravity: 70th anniversary of the unsolved problem", Phys. Usp., 48, 1039-1053 (2005). [DOI]. (Cited on page 6.)

[139] Greensite, J., "Is there a minimum length in $D=4$ lattice quantum gravity?", Phys. Lett. B, 255, 375-380 (1991). [DOI]. (Cited on page 44.)

[140] Gross, D.J. and Mende, P.F., "String theory beyond the Planck scale", Nucl. Phys. B, 303, 407-454 (1988). [DOI]. (Cited on pages 10 and 24.)

[141] Hagar, A., "Length Matters: The History and the Philosophy of the Notion of Fundamental Length in Modern Physics", in preparation, (2012). Online version (accessed 17 December 2012): http://mypage.iu.edu/ hagara/LMBOOK.pdf. (Cited on page 10.)

[142] Harbach, U. and Hossenfelder, S., "The Casimir effect in the presence of a minimal length", Phys. Lett. B, 632, 379-383 (2006). [DOI], [arXiv:hep-th/0502142 [hep-th]]. (Cited on page 59.)

[143] Harbach, U., Hossenfelder, S., Bleicher, M. and Stoecker, H., "Probing the minimal length scale by precision tests of the muon g-2", Phys. Lett. B, 584, 109-113 (2004). [DOI], [arXiv:hep-ph/0308138 [hep-ph]]. (Cited on page 59.)

[144] Hassan, S.F. and Sloth, M.S., "Trans-Planckian effects in inflationary cosmology and the modified uncertainty principle", Nucl. Phys. B, 674, 434-458 (2003). [DOI], [arXiv:hep-th/0204110 [hep-th]]. (Cited on page 9.)

[145] Hawking, S.W., "Particle creation by black holes", Commun. Math. Phys., 43, 199-220 (1975). [DOI]. (Cited on page 9.)

[146] Heisenberg, W., The Physical Principles of the Quantum Theory, (University of Chicago Press, Chicago, 1930). [Google Books]. (Cited on page 11.)

[147] Heisenberg, W., "Zur Theorie der 'Schauer' in der Höhenstrahlung”, Z. Phys., 101, 533-540 (1936). [DOI]. (Cited on page 7.)

[148] Heisenberg, W., "Über die in der Theorie der Elementarteilchen auftretende universelle Länge", Ann. Phys. (Leipzig), 32, 20-33 (1938). [DOI]. (Cited on pages 7 and 8.)

[149] Heisenberg, W., "Bericht über die allgemeinen Eigenschaften der Elementarteilchen / Report on the General Properties of Elementary Particles", in Blum, W., Dürr, H.-P. and Rechenberg, H., eds., Werner Heisenberg: Gesammelte Werke. Collected Works, Series B, pp. 346-358, (Springer, Berlin; New York, 1984). (Cited on page 8.)

[150] Heisenberg, W., "[247] Heisenberg an Peierls, 1930", in von Meyenn, K., ed., Wissenschaftlicher Briefwechsel mit Bohr, Einstein, Heisenberg u.a., Bd. II: 1930-1939, Sources in the History of Mathematics and Physical Sciences, 6, pp. 15-18, (Springer, Berlin; New York, 1985). [Google Books]. (Cited on page 8.)

[151] Hinchliffe, I., Kersting, N. and Ma, Y.L., "Review of the phenomenology of noncommutative geometry", Int. J. Mod. Phys. A, 19, 179-204 (2004). [DOI], [arXiv:hep-ph/0205040 [hep-ph]]. (Cited on pages 41 and 60.)

[152] Hossenfelder, S., "Running coupling with minimal length", Phys. Rev. D, 70, 105003 (2004). [DOI], [arXiv:hep-ph/0405127 [hep-ph]]. (Cited on page 61.)

[153] Hossenfelder, S., "Suppressed black hole production from minimal length", Phys. Lett. B, 598, 92-98 (2004). [DOI], [arXiv:hep-th/0404232 [hep-th]]. (Cited on page 61.) 
[154] Hossenfelder, S., "Interpretation of quantum field theories with a minimal length scale", Phys. Rev. D, 73, 105013 (2006). [DOI], [arXiv:hep-th/0603032 [hep-th]]. (Cited on page 47.)

[155] Hossenfelder, S., "Self-consistency in theories with a minimal length", Class. Quantum Grav., 23, 1815-1821 (2006). [DOI], [arXiv:hep-th/0510245 [hep-th]]. (Cited on pages 50 and 58.)

[156] Hossenfelder, S., "Deformed Special Relativity in Position Space", Phys. Lett. B, 649, 310-316 (2007). [DOI], [arXiv:gr-qc/0612167 [gr-qc]]. (Cited on pages 48 and 58.)

[157] Hossenfelder, S., "Multi-Particle States in Deformed Special Relativity", Phys. Rev. D, 75, 105005 (2007). [DOI], [arXiv:hep-th/0702016 [hep-th]]. (Cited on pages 56 and 61.)

[158] Hossenfelder, S., "A note on quantum field theories with a minimal length scale", Class. Quantum Grav., 25, 038003 (2008). [DOI], [arXiv:0712.2811 [hep-th]]. (Cited on page 50.)

[159] Hossenfelder, S., "The Box-Problem in Deformed Special Relativity", arXiv, e-print, (2009). [arXiv:0912.0090 [gr-qc]]. (Cited on page 58.)

[160] Hossenfelder, S., "Bounds on an energy-dependent and observer-independent speed of light from violations of locality", Phys. Rev. Lett., 104, 140402 (2010). [DOI], [arXiv:1004.0418 [hep-ph]]. (Cited on page 58.)

[161] Hossenfelder, S., "Comment on arXiv:1007.0718 by Lee Smolin", arXiv, e-print, (2010). [arXiv:1008.1312 [gr-qc]]. (Cited on page 58.)

[162] Hossenfelder, S., "Comments on Nonlocality in Deformed Special Relativity, in reply to arXiv:1004.0664 by Lee Smolin and arXiv:1004.0575 by Jacob et al", arXiv, e-print, (2010). [arXiv:1005.0535 [gr-qc]]. (Cited on page 58.)

[163] Hossenfelder, S., "Reply to arXiv:1006.2126 by Giovanni Amelino-Camelia et al", arXiv, e-print, (2010). [arXiv:1006.4587 [gr-qc]]. (Cited on page 58.)

[164] Hossenfelder, S., "Experimental Search for Quantum Gravity", in Frignanni, V.R., ed., Classical and Quantum Gravity: Theory, Analysis and Applications, (Nova Science Publishers, Hauppauge, NY, 2011). [arXiv:1010.3420 [gr-qc]]. (Cited on page 66.)

[165] Hossenfelder, S., "Can we measure structures to a precision better than the Planck length?", Class. Quantum Grav., 29, 115011 (2012). [DOI], [arXiv:1205.3636 [gr-qc]]. (Cited on page 22.)

[166] Hossenfelder, S., 'Comment on arXiv:1104.2019, 'Relative locality and the soccer ball problem,' by Amelino-Camelia et al", arXiv, e-print, (2012). [arXiv:1202.4066 [hep-th]]. (Cited on page 56.)

[167] Hossenfelder, S., Bleicher, M., Hofmann, S., Ruppert, J., Scherer, S. and Stöcker, H., "Signatures in the Planck regime", Phys. Lett. B, 575, 85-99 (2003). [DOI], [arXiv:hep-th/0305262 [hep-th]]. (Cited on pages 59, 60, and 61.)

[168] Hsu, S.D.H., "Quantum production of black holes", Phys. Lett. B, 555, 92-98 (2003). [DOI], [arXiv:hep-ph/0203154]. (Cited on page 13.)

[169] Jacob, U., Mercati, F., Amelino-Camelia, G. and Piran, T., "Modifications to Lorentz invariant dispersion in relatively boosted frames", Phys. Rev. D, 82, 084021 (2010). [DOI], [arXiv:1004.0575 [astro-ph.HE]]. (Cited on pages 48, 57, 58, and 62.)

[170] Johnson, C.V., "D-Brane Primer", in Harvey, J., Kachru, S. and Silverstein, E., eds., Strings, Branes and Gravity (TASI 99), Boulder, Colorado, USA, 31 May-25 June 1999, pp. 129-350, (World Scientific, Singapore; River Edge, NJ, 2000). [DOI], [arXiv:hep-th/0007170 [hep-th]], [Google Books]. (Cited on page 27.)

[171] Judes, S. and Visser, M., "Conservation laws in 'Doubly special relativity"', Phys. Rev. D, 68, 045001 (2003). [DOI], [arXiv:gr-qc/0205067 [gr-qc]]. (Cited on pages 56 and 62.) 
[172] Kalyana Rama, S., "Some consequences of the generalized uncertainty principle: Statistical mechanical, cosmological, and varying speed of light", Phys. Lett. B, 519, 103-110 (2001). [DOI], [arXiv:hep-th/0107255 [hep-th]]. (Cited on page 62.)

[173] Karliner, M., Klebanov, I.R. and Susskind, L., "Size and shape of strings", Int. J. Mod. Phys. A, 3, 1981 (1988). [DOI]. (Cited on page 25.)

[174] Kempf, A., "Quantum groups and quantum field theory with nonzero minimal uncertainties in positions and momenta", Czech. J. Phys., 44, 1041-1048 (1994). [DOI], [arXiv:hep-th/9405067]. (Cited on pages 9 and 60.)

[175] Kempf, A., "Uncertainty relation in quantum mechanics with quantum group symmetry", J. Math. Phys., 35, 4483-4496 (1994). [DOI], [arXiv:hep-th/9311147]. (Cited on pages 9, 48, and 50.)

[176] Kempf, A., "On Noncommutative Geometric Regularisation", Phys. Rev. D, 54, 5174-5178 (1996). [DOI], [arXiv:hep-th/9602119]. (Cited on page 60.)

[177] Kempf, A., "Non-pointlike particles in harmonic oscillators", J. Phys. A: Math. Gen., 30, 2093-2102 (1997). [DOI], [arXiv:hep-th/9604045 [hep-th]]. (Cited on page 59.)

[178] Kempf, A., "On quantum field theory with nonzero minimal uncertainties in positions and momenta", J. Math. Phys., 38, 1347-1372 (1997). [DOI], [arXiv:hep-th/9602085]. (Cited on pages 9 and 60.)

[179] Kempf, A., "Fields over unsharp coordinates", Phys. Rev. Lett., 85, 2873 (2000). [DOI], [arXiv:hepth/9905114 [hep-th]]. (Cited on page 65.)

[180] Kempf, A., "Mode generating mechanism in inflation with cutoff", Phys. Rev. D, 63, 083514 (2001). [DOI], [arXiv:astro-ph/0009209]. (Cited on page 9.)

[181] Kempf, A., "Covariant Information-Density Cutoff in Curved Space-Time", Phys. Rev. Lett., 92, 221301 (2004). [DOI], [arXiv:gr-qc/0310035]. (Cited on page 65.)

[182] Kempf, A., "Spacetime could be simultaneously continuous and discrete in the same way that information can", New J. Phys., 12, 115001 (2010). [DOI], [arXiv:1010.4354 [gr-qc]]. (Cited on page 65.)

[183] Kempf, A. and Mangano, G., "Minimal length uncertainty relation and ultraviolet regularization", Phys. Rev. D, 55, 7909-7920 (1997). [DOI], [arXiv:hep-th/9612084 [hep-th]]. (Cited on page 60.)

[184] Kempf, A., Mangano, G. and Mann, R.B., "Hilbert space representation of the minimal length uncertainty relation", Phys. Rev. D, 52, 1108-1118 (1995). [DOI], [arXiv:hep-th/9412167]. (Cited on pages 9,50 , and 59.)

[185] Kim, W., Kim, Y.-W. and Park, Y.-J., "Entropy of the Randall-Sundrum brane world with the generalized uncertainty principle", Phys. Rev. D, 74, 104001 (2006). [DOI], [arXiv:gr-qc/0605084 [gr-qc]]. (Cited on page 64.)

[186] Kim, W., Son, E.J. and Yoon, M., "Thermodynamics of a black hole based on a generalized uncertainty principle", J. High Energy Phys., 2008(01), 035 (2008). [DOI], [arXiv:0711.0786 [gr-qc]]. (Cited on page 64.)

[187] Kiritsis, E., Introduction to Superstring Theory, Leuven Notes in Mathematical and Theoretical Physics, (Leuven University Press, Leuven, 1998). [arXiv:hep-th/9709062]. (Cited on page 22.)

[188] Kober, M., "Gauge Theories under Incorporation of a Generalized Uncertainty Principle", Phys. Rev. D, 82, 085017 (2010). [DOI], [arXiv:1008.0154 [physics.gen-ph]]. (Cited on pages 60 and 61.)

[189] Kober, M., "Electroweak Theory with a Minimal Length", Int. J. Mod. Phys. A, 26, 4251-4285 (2011). [DOI], [arXiv:1104.2319 [hep-ph]]. (Cited on page 61.) 
[190] Kober, M., "Generalized Quantization Principle in Canonical Quantum Gravity and Application to Quantum Cosmology", Int. J. Mod. Phys. A, 27, 1250106 (2012). [DOI], [arXiv:1109.4629 [gr-qc]]. (Cited on page 61.)

[191] Kostelecky, V.Alan and Russell, N., "Data Tables for Lorentz and CPT Violation", Rev. Mod. Phys., 83, 11 (2011). [DOI], [arXiv:0801.0287 [hep-ph]]. (Cited on page 62.)

[192] Kothawala, D., Sriramkumar, L., Shankaranarayanan, S. and Padmanabhan, T., "Path integral duality modified propagators in spacetimes with constant curvature", Phys. Rev. D, 80, 044005 (2009). [DOI], [arXiv:0904.3217 [hep-th]]. (Cited on page 64.)

[193] Kowalski-Glikman, J., "Observer independent quantum of mass", Phys. Lett. A, 286, 391-394 (2001). [DOI], [arXiv:hep-th/0102098 [hep-th]]. (Cited on page 46.)

[194] Kowalski-Glikman, J., "Doubly special quantum and statistical mechanics from quantum $\kappa$ - Poincaré algebra", Phys. Lett. A, 299, 454-460 (2002). [DOI], [arXiv:hep-th/0111110 [hep-th]]. (Cited on page 62.)

[195] Kowalski-Glikman, J., "Introduction to Doubly Special Relativity", in Amelino-Camelia, G. and Kowalski-Glikman, J., eds., Planck Scale Effects in Astrophysics and Cosmology, 40th Karpacz Winter School of Theoretical Physics, Ladek Zdrój, Poland, 4-14 February 2004, Lecture Notes in Physics, 669, pp. 131-159, (Springer, Berlin; New York, 2005). [DOI], [arXiv:hep-th/0405273 [hepth]]. (Cited on page 56.)

[196] Kowalski-Glikman, J., "An introduction to relative locality", unpublished, (2013). (Cited on page 62.)

[197] Kowalski-Glikman, J. and Nowak, S., "Doubly special relativity and de Sitter space", Class. Quantum Grav., 20, 4799-4816 (2003). [DOI], [arXiv:hep-th/0304101 [hep-th]]. (Cited on pages 54, 55, and 56.)

[198] Kowalski-Glikman, J. and Starodubtsev, A., "Effective particle kinematics from Quantum Gravity", Phys. Rev. D, 78, 084039 (2008). [DOI], [arXiv:0808.2613 [gr-qc]]. (Cited on page 66.)

[199] Kragh, H., "Arthur March, Werner Heisenberg, and the search for a smallest length", Rev. Hist. Sci., 48, 401-434 (1995). [DOI]. (Cited on page 8.)

[200] Lévi, R., "Théorie de l'action universelle et discontinue", J. Phys. Radium, 8, 182-198 (1927). [DOI]. (Cited on page 6.)

[201] Li, X., "Black hole entropy without brick walls", Phys. Lett. B, 540, 9-13 (2002). [DOI], [arXiv:grqc/0204029 [gr-qc]]. (Cited on page 64.)

[202] Litim, D.F., "Fixed Points of Quantum Gravity and the Renormalisation Group", in From Quantum to Emergent Gravity: Theory and Phenomenology, June 11-15 2007, Trieste, Italy, Proceedings of Science, PoS(QG-Ph)024, (SISSA, Trieste, 2008). [arXiv:0810.3675 [hep-th]]. URL (accessed 15 November 2012):

http://pos.sissa.it/cgi-bin/reader/conf.cgi?confid=43. (Cited on page 38.)

[203] Loll, R., "Discrete Approaches to Quantum Gravity in Four Dimensions", Living Rev. Relativity, 1, lrr-1998-13 (1998). [arXiv:gr-qc/9805049 [gr-qc]]. URL (accessed 11 October 2011):

http://www.livingreviews.org/lrr-1998-13. (Cited on page 44.)

[204] Lowe, D.A., Polchinski, J., Susskind, L., Thorlacius, L. and Uglum, J., "Black hole complementarity versus locality", Phys. Rev. D, 52, 6997-7010 (1995). [DOI], [arXiv:hep-th/9506138]. (Cited on page 25.)

[205] Lukierski, J., Nowicki, A. and Ruegg, H., "New quantum Poincaré algebra and $\kappa$-deformed field theory", Phys. Lett. B, 293, 344-352 (1992). [DOI]. (Cited on page 54.) 
[206] Lukierski, J., Ruegg, H. and Zakrzewski, W.J., "Classical and Quantum Mechanics of Free $\kappa$ Relativistic Systems", Ann. Phys. (N.Y.), 243, 90-116 (1995). [DOI], [arXiv:hep-th/9312153 [hepth]]. (Cited on page 65.)

[207] Maggiore, M., "The Algebraic structure of the generalized uncertainty principle", Phys. Lett. B, 319, 83-86 (1993). [DOI], [arXiv:hep-th/9309034 [hep-th]]. (Cited on page 48.)

[208] Maggiore, M., "A generalized uncertainty principle in quantum gravity", Phys. Lett. B, 304, 65-69 (1993). [DOI], [arXiv:hep-th/9301067]. (Cited on page 18.)

[209] Maggiore, M., "Quantum groups, gravity and the generalized uncertainty principle", Phys. Rev. D, 49, 5182-5187 (1994). [DOI], [arXiv:hep-th/9305163 [hep-th]]. (Cited on page 48.)

[210] Magueijo, J., "Could quantum gravity be tested with high intensity lasers?", Phys. Rev. D, 73, 124020 (2006). [DOI], [arXiv:gr-qc/0603073 [gr-qc]]. (Cited on page 56.)

[211] Magueijo, J. and Smolin, L., "Lorentz invariance with an invariant energy scale", Phys. Rev. Lett., 88, 190403 (2002). [DOI], [arXiv:hep-th/0112090 [hep-th]]. (Cited on page 61.)

[212] Magueijo, J. and Smolin, L., "Generalized Lorentz invariance with an invariant energy scale", Phys. Rev. D, 67, 044017 (2003). [DOI], [arXiv:gr-qc/0207085 [gr-qc]]. (Cited on pages 46 and 56.)

[213] Majid, S. and Ruegg, H., "Bicrossproduct structure of $\kappa$-Poincare group and non-commutative geometry", Phys. Lett. B, 334, 348-354 (1994). [DOI], [arXiv:hep-th/9405107]. (Cited on pages 9, 54, and 55.)

[214] Majumder, B., "Black Hole Entropy and the Modified Uncertainty Principle: A heuristic analysis", Phys. Lett. B, 703, 402-405 (2011). [DOI], [arXiv:1106.0715 [gr-qc]]. (Cited on page 64.)

[215] Majumder, B., "Effects of GUP in Quantum Cosmological Perfect Fluid Models", Phys. Lett. B, 699, 315-319 (2011). [DOI], [arXiv:1104.3488 [gr-qc]]. (Cited on page 64.)

[216] Majumder, B., "The Generalized Uncertainty Principle and the Friedmann equations", Astrophys. Space Sci., 336, 331-335 (2011). [DOI], [arXiv:1105.2425 [gr-qc]]. (Cited on page 64.)

[217] Majumder, B., "Quantum Black Hole and the Modified Uncertainty Principle", Phys. Lett. B, 701, 384-387 (2011). [DOI], [arXiv:1105.5314 [gr-qc]]. (Cited on page 64.)

[218] Manrique, E., Rechenberger, S. and Saueressig, F., "Asymptotically Safe Lorentzian Gravity", Phys. Rev. Lett., 106, 251302 (2011). [DOI], [arXiv:1102.5012 [hep-th]]. (Cited on page 68.)

[219] March, A., "Die Geometrie kleinster Räume", Z. Phys., 104, 93 (1936). (Cited on pages 6 and 64.)

[220] Martin, J. and Brandenberger, R.H., "Trans-Planckian problem of inflationary cosmology", Phys. Rev. D, 63, 123501 (2001). [DOI], [arXiv:hep-th/0005209]. (Cited on pages 9 and 67.)

[221] Maziashvili, M., "Implications of minimum-length deformed quantum mechanics for QFT/QG", Fortschr. Phys. (2013). [DOI], [arXiv:1110.0649 [gr-qc]]. (Cited on page 61.)

[222] Mead, C.A., "Possible Connection Between Gravitation and Fundamental Length", Phys. Rev., 135, B849-B862 (1964). [DOI]. (Cited on pages 9, 11, 14, 15, 17, and 68.)

[223] Mead, C.A., "Observable Consequences of Fundamental-Length Hypotheses", Phys. Rev., 143, 9901005 (1966). [DOI]. (Cited on page 9.)

[224] Mead, C.A. and Wilczek, F., "Walking the Planck Length through History", Phys. Today, 54, 15 (2001). [DOI]. (Cited on page 9.)

[225] Medved, A.J.M. and Vagenas, E.C., "When conceptual worlds collide: The GUP and the BH entropy", Phys. Rev. D, 70, 124021 (2004). [DOI], [arXiv:hep-th/0411022 [hep-th]]. (Cited on page 64.) 
[226] Meljanac, S. and Samsarov, A., "Scalar field theory on $\kappa$-Minkowski space-time and translation and Lorentz invariance", Int. J. Mod. Phys. A, 26, 1439-1468 (2011). [DOI], [arXiv:1007.3943 [hep-th]]. (Cited on page 60.)

[227] Mena Marugán, G.A., Olmedo, J. and Pawlowski, T., "Prescriptions in Loop Quantum Cosmology: A comparative analysis", Phys. Rev. D, 84, 064012 (2011). [DOI], [arXiv:1108.0829 [gr-qc]]. (Cited on page 34.)

[228] Mende, P.F. and Ooguri, H., "Borel summation of string theory for Planck scale scattering", Nucl. Phys. B, 339, 641-662 (1990). [DOI]. (Cited on page 24.)

[229] Mercuri, S., "Introduction to Loop Quantum Gravity", in Pinheiro, C., de Arruda, A.S., Blas, H. and Pires, G.O., eds., 5th International School on Field Theory and Gravitation, April 20-24, 2009, Cuiabá, Brazil, Proceedings of Science, PoS(ISFTG)016, (SISSA, Trieste, 2009). [arXiv:1001.1330 [gr-qc]]. URL (accessed 15 November 2012):

http://pos.sissa.it/cgi-bin/reader/conf.cgi?confid=81. (Cited on page 32.)

[230] Mignemi, S., "Doubly special relativity and translation invariance", Phys. Lett. B, 672, 186-189 (2009). [DOI], [arXiv:0808.1628 [gr-qc]]. (Cited on page 53.)

[231] Mimasu, K. and Moretti, S., "Top quark phenomenology of the Arkani-Hamed-Dimopoulos-Dvali model and the minimal length scenario", Phys. Rev. D, 85, 074019 (2012). [DOI], [arXiv:1108.3280 [hep-ph]]. (Cited on page 61.)

[232] Moayedi, S.K., Setare, M.R. and Moayeri, H., "Quantum Gravitational Corrections to the Real Klein-Gordon Field in the Presence of a Minimal Length", Int. J. Theor. Phys., 49, 2080-2088 (2010). [DOI], [arXiv:1004.0563 [hep-th]]. (Cited on page 60.)

[233] Moayedi, S.K., Setare, M.R. and Moayeri, H., "Formulation of the Spinor Field in the Presence of a Minimal Length Based on the Quesne-Tkachuk Algebra", Int. J. Mod. Phys. A, 26, 4981-4990 (2011). [DOI], [arXiv:1105.1900 [hep-th]]. (Cited on page 60.)

[234] Möglich, F., "Über das Massenverhältnis Elektron-Neutron", Die Naturwissenschaften, 26, 409-410 (1938). [DOI]. (Cited on page 6.)

[235] Mohaupt, T., "Introduction to string theory", in Giulini, D., Kiefer, C. and Lämmerzahl, C., eds., Quantum Gravity: From Theory to Experimental Search, 271th WE-Heraeus Seminar 'Aspects of Quantum Gravity', Bad Honnef, Germany, 24 February-1 March 2002, Lecture Notes in Physics, 631, pp. 173-251, (Springer, Berlin; New York, 2003). [DOI], [arXiv:hep-th/0207249]. (Cited on page 22.)

[236] Moyal, J.E., "Quantum mechanics as a statistical theory", Proc. Cambridge Philos. Soc., 45, 99-124 (1949). [DOI]. (Cited on page 42.)

[237] Myung, Y.S., Kim, Y.-W. and Park, Y.-J., "Black hole thermodynamics with generalized uncertainty principle", Phys. Lett. B, 645, 393-397 (2007). [DOI], [arXiv:gr-qc/0609031 [gr-qc]]. (Cited on page 64.)

[238] Ng, Y.J. and van Dam, H., "Limitation to quantum measurements of space-time distances", Ann. N.Y. Acad. Sci., 755, 579-584 (1995). [DOI], [arXiv:hep-th/9406110]. (Cited on page 20.)

[239] Nicolini, P., "Noncommutative Black Holes, The Final Appeal To Quantum Gravity: A Review", Int. J. Mod. Phys. A, 24, 1229-1308 (2009). [DOI], [arXiv:0807.1939 [hep-th]]. (Cited on page 66.)

[240] Niedermaier, M. and Reuter, M., "The Asymptotic Safety Scenario in Quantum Gravity", Living Rev. Relativity, 9, lrr-2006-5 (2006). URL (accessed 20 January 2012):

http://www.livingreviews.org/lrr-2006-5. (Cited on page 38.) 
[241] Nouicer, K., "Casimir effect in the presence of minimal lengths", J. Phys. A: Math. Gen., 38, 1002710035 (2005). [DOI], [arXiv:hep-th/0512027 [hep-th]]. (Cited on page 59.)

[242] Nozari, K. and Fazlpour, B., "Generalized uncertainty principle, modified dispersion relations and early universe thermodynamics", Gen. Relativ. Gravit., 38, 1661-1679 (2006). [DOI], [arXiv:grqc/0601092 [gr-qc]]. (Cited on page 62.)

[243] Nozari, K. and Mehdipour, S.H., "Gravitational uncertainty and black hole remnants", Mod. Phys. Lett. A, 20, 2937-2948 (2005). [DOI], [arXiv:0809.3144 [gr-qc]]. (Cited on page 64.)

[244] Nozari, K. and Pedram, P., "Minimal length and bouncing-particle spectrum", Europhys. Lett., 92, 50013 (2010). [DOI], [arXiv:1011.5673 [hep-th]]. (Cited on page 59.)

[245] Nozari, K., Pedram, P. and Molkara, M., "Minimal Length, Maximal Momentum and the Entropic Force Law", Int. J. Theor. Phys., 51, 1268-1275 (2012). [DOI], [arXiv:1111.2204 [gr-qc]]. (Cited on page 64.)

[246] Olmo, G.J., "Palatini Actions and Quantum Gravity Phenomenology", J. Cosmol. Astropart. Phys., 2011(10), 018 (2011). [DOI], [arXiv:1101.2841 [gr-qc]]. (Cited on page 56.)

[247] Padmanabhan, T., "Physical Significance of Planck Length", Ann. Phys. (N.Y.), 165, 38-58 (1985). [DOI]. (Cited on pages 36 and 63.)

[248] Padmanabhan, T., "Planck length as the lower bound to all physical length scales", Gen. Relativ. Gravit., 17, 215-221 (1985). [DOI]. (Cited on page 36.)

[249] Padmanabhan, T., "Limitations on the operational definition of space-time events and quantum gravity", Class. Quantum Grav., 4, L107-L113 (1987). [DOI]. (Cited on page 63.)

[250] Padmanabhan, T., "Duality and zero point length of space-time", Phys. Rev. Lett., 78, 1854-1857 (1997). [DOI], [arXiv:hep-th/9608182 [hep-th]]. (Cited on page 63.)

[251] Padmanabhan, T., "Hypothesis of path integral duality. I. Quantum gravitational corrections to the propagator", Phys. Rev. D, 57, 6206-6215 (1998). [DOI]. (Cited on page 63.)

[252] Panella, O., "Casimir-Polder intermolecular forces in minimal length theories", Phys. Rev. D, 76, 045012 (2007). [DOI], [arXiv:0707.0405 [hep-th]]. (Cited on page 59.)

[253] Panes, B., "Minimum length-maximum velocity", Eur. Phys. J. C, 72, 1930 (2012). [DOI], [arXiv:1112.3753 [hep-ph]]. (Cited on page 49.)

[254] Pauli, W., "[899] Pauli an Heisenberg, 11. Juli 1947", in von Meyenn, K., ed., Wolfgang Pauli. Wissenschaftlicher Briefwechsel mit Bohr, Einstein, Heisenberg u.a, Bd. III: 1940-1949, Sources in the History of Mathematics and Physical Sciences, 11, pp. 461-464, (Springer, Berlin; New York, 1993). Online version (accessed 12 January 2012):

http://cdsweb.cern.ch/record/84589. (Cited on page 8.)

[255] Pedram, P., "A higher order GUP with minimal length uncertainty and maximal momentum", Phys. Lett. B, 714, 317-323 (2011). [DOI], [arXiv:1110.2999 [hep-th]]. (Cited on page 59.)

[256] Pedram, P., "Minimal Length and the Quantum Bouncer: A Nonperturbative Study", Int. J. Theor. Phys., 51, 1901-1910 (2012). [DOI], [arXiv:1201.2802 [hep-th]]. (Cited on page 59.)

[257] Pedram, P., "New Approach to Nonperturbative Quantum Mechanics with Minimal Length Uncertainty", Phys. Rev. D, 85, 024016 (2012). [DOI], [arXiv:1112.2327 [hep-th]]. (Cited on pages 59 and 62.)

[258] Pedram, P., "A note on the one-dimensional hydrogen atom with minimal length uncertainty", arXiv, e-print, (2012). [arXiv:1203.5478 [quant-ph]]. (Cited on page 59.) 
[259] Pedram, P., Nozari, K. and Taheri, S.H., "The effects of minimal length and maximal momentum on the transition rate of ultra cold neutrons in gravitational field", J. High Energy Phys., 2011(03), 093 (2011). [DOI], [arXiv:1103.1015 [hep-th]]. (Cited on page 59.)

[260] Percacci, R., "Asymptotic Safety", in Oriti, D., ed., Approaches to Quantum Gravity: Towards a New Understanding of Space, Time and Matter, pp. 111-128, (Cambridge University Press, Cambridge; New York, 2009). [arXiv:0709.3851 [hep-th]]. (Cited on page 38.)

[261] Percacci, R. and Vacca, G.P., "Asymptotic Safety, Emergence and Minimal Length", Class. Quantum Grav., 27, 245026 (2010). [DOI], [arXiv:1008.3621 [hep-th]]. (Cited on pages 38, 39, 40, and 57.)

[262] Peres, A. and Rosen, N., "Quantum Limitations on the Measurement of Gravitational Fields", Phys. Rev., 118, 335-336 (1960). [DOI]. (Cited on page 8.)

[263] Pérez-Payán, S., Sabido, M. and Yee, C., "Effects of deformed phase space on scalar field cosmology", arXiv, e-print, (2011). [arXiv:1111.6136 [hep-th]]. (Cited on page 65.)

[264] Pikovski, I., Vanner, M.R., Aspelmeyer, M., Kim, M.S. and Brukner, Č., "Probing Planck-scale physics with quantum optics", Nature Phys., 8, 393-397 (2012). [DOI], [ADS], [arXiv:1111.1979 [quant-ph]]. (Cited on pages 62 and 67.)

[265] Planck, M., "Über irreversible Strahlungsvorgänge", Ann. Phys. (Berlin), 1, 69 (1900). [DOI]. (Cited on page 6.)

[266] Pokrowski, G.I., "Zur Frage nach der Struktur der Zeit", Z. Phys., 51, 737-739 (1928). [DOI]. (Cited on page 6.)

[267] Proca, A. and Goudsmit, S., "Sur la masse des particules élémentaires", J. Phys. Radium, 10, 209-214 (1939). [DOI]. (Cited on page 6.)

[268] Quesne, C. and Tkachuk, V.M., "Composite system in deformed space with minimal length", Phys. Rev. A, 81, 012106 (2010). [DOI], [arXiv:0906.0050 [hep-th]]. (Cited on page 62.)

[269] Raghavan, R.S., "Time-Energy Uncertainty in Neutrino Resonance: Quest for the Limit of Validity of Quantum Mechanics", arXiv, e-print, (2009). [arXiv:0907.0878 [hep-ph]]. (Cited on page 65.)

[270] Reuter, M. and Schwindt, J.-M., "A minimal length from the cutoff modes in asymptotically safe quantum gravity", J. High Energy Phys., 2006(01), 070 (2006). [DOI], [arXiv:hep-th/0511021 [hepth]]. (Cited on page 39.)

[271] Rovelli, C. and Smolin, L., "Discreteness of area and volume in quantum gravity", Nucl. Phys. B, 442, 593-619 (1995). [DOI], [arXiv:gr-qc/9411005]. (Cited on page 34.)

[272] Rychkov, V.S., "Observers and measurements in noncommutative space-times", J. Cosmol. Astropart. Phys., 2003(07), 006 (2003). [DOI], [arXiv:hep-th/0305187 [hep-th]]. (Cited on page 57.)

[273] Said, J.L. and Adami, K.Z., "The generalized uncertainty principle in $f(R)$ gravity for a charged black hole", Phys. Rev. D, 83, 043008 (2011). [DOI], [arXiv:1102.3553 [gr-qc]]. (Cited on page 64.)

[274] Salecker, H. and Wigner, E.P., "Quantum limitations of the measurement of space-time distances", Phys. Rev., 109, 571-577 (1958). [DOI]. (Cited on page 16.)

[275] Scardigli, F., "Generalized uncertainty principle in quantum gravity from micro-black hole gedanken experiment", Phys. Lett., B452, 39-44 (1999). [DOI], [arXiv:hep-th/9904025]. (Cited on page 13.)

[276] Scardigli, F. and Casadio, R., "Generalized uncertainty principle, extra dimensions and holography", Class. Quantum Grav., 20, 3915-3926 (2003). [DOI], [arXiv:hep-th/0307174 [hep-th]]. (Cited on page 64.) 
[277] Schutzhold, R. and Unruh, W.G., "Large-scale nonlocality in 'doubly special relativity' with an energy-dependent speed of light", JETP Lett., 78, 431-435 (2003). [DOI], [arXiv:gr-qc/0308049 [grqc]]. (Cited on page 58.)

[278] Schwarz, J.H., "Introduction to superstring theory", arXiv, e-print, (2000). [arXiv:hep-ex/0008017]. (Cited on page 22.)

[279] Sefiedgar, A.S., Nozari, K. and Sepangi, H.R., "Modified dispersion relations in extra dimensions", Phys. Lett. B, 696, 119-123 (2011). [DOI], [arXiv:1012.1406 [gr-qc]]. (Cited on page 64.)

[280] Setare, M.R., "Corrections to the Cardy-Verlinde formula from the generalized uncertainty principle", Phys. Rev. D, 70, 087501 (2004). [DOI], [arXiv:hep-th/0410044 [hep-th]]. (Cited on page 64.)

[281] Setare, M.R., "The generalized uncertainty principle and corrections to the Cardy-Verlinde formula in SAdS 5 black holes", Int. J. Mod. Phys. A, 21, 1325-1332 (2006). [DOI], [arXiv:hep-th/0504179 [hep-th]]. (Cited on page 64.)

[282] Shankaranarayanan, S. and Padmanabhan, T., "Hypothesis of path integral duality: Applications to QED", Int. J. Mod. Phys. D, 10, 351-366 (2001). [DOI], [arXiv:gr-qc/0003058 [gr-qc]]. (Cited on page 64 .)

[283] Shenker, S.H., "Another Length Scale in String Theory?", arXiv, e-print, (1995). [arXiv:hepth/9509132]. (Cited on page 27.)

[284] Sindoni, L., "Emergent Models for Gravity: an Overview of Microscopic Models", SIGMA, 8, 027 (2012). [DOI], [arXiv:1110.0686 [gr-qc]]. URL (accessed 20 November 2012): http://sigma-journal.com/2012/027/. (Cited on page 44.)

[285] Smailagic, A., Spallucci, E. and Padmanabhan, T., "String theory T-duality and the zero point length of spacetime", arXiv, e-print, (2003). [arXiv:hep-th/0308122]. (Cited on pages 31 and 32.)

[286] Smolin, L., "On limitations of the extent of inertial frames in non-commutative relativistic spacetimes", arXiv, e-print, (2010). [arXiv:1007.0718 [gr-qc]]. (Cited on pages 48, 57, 58, and 62.)

[287] Smolin, L., "Classical paradoxes of locality and their possible quantum resolutions in deformed special relativity", Gen. Relativ. Gravit., 43, 3671-3691 (2011). [DOI], [arXiv:1004.0664 [gr-qc]]. (Cited on pages $48,54,57,58$, and 62.$)$

[288] Snyder, H.S., "Quantized Space-Time", Phys. Rev., 71, 38-41 (1947). [DOI]. (Cited on pages 8 and 52.)

[289] Snyder, H.S., "[817] Snyder an Pauli, 1946", in von Meyenn, K., ed., Wissenschaftlicher Briefwechsel mit Bohr, Einstein, Heisenberg u.a., Bd. III: 1940-1949, Sources in the History of Mathematics and Physical Sciences, 11, pp. 358-360, (Springer, Berlin; New York, 1993). (Cited on page 8.)

[290] Sorkin, R.D., "Causal sets: Discrete gravity", in Gomberoff, A. and Marolf, D., eds., Lectures on Quantum Gravity, Pan-American Advanced Studies Institute School on Quantum Gravity, held at the CECS, Valdivia, Chile, January 4-14, 2002, Series of the Centro de Estudios Científicos, pp. 305-327, (Springer, New York, 2005). [arXiv:gr-qc/0309009 [gr-qc]]. (Cited on page 44.)

[291] Spallucci, E. and Fontanini, M., "Zero-point Length, Extra-Dimensions and String T-duality", in Grece, S.A., ed., New Developments in String Theory Research, pp. 245-270, (Nova Science Publishers, Hauppauge, NY, 2005). [arXiv:gr-qc/0508076]. (Cited on page 31.)

[292] Sprenger, M., Nicolini, P. and Bleicher, M., "Neutrino oscillations as a novel probe for a minimal length", Class. Quantum Grav., 28, 235019 (2011). [DOI], [arXiv:1011.5225 [hep-ph]]. (Cited on page 59.) 
[293] Sprenger, M., Nicolini, P. and Bleicher, M., "Physics on the smallest scales: an introduction to minimal length phenomenology", Eur. J. Phys., 33, 853-862 (2012). [DOI], [arXiv:1202.1500 [physics.edph]]. (Cited on page 66.)

[294] Srinivasan, K., Sriramkumar, L. and Padmanabhan, T., "Hypothesis of path integral duality. II. Corrections to quantum field theoretic results", Phys. Rev. D, 58, 044009 (1998). [DOI], [arXiv:grqc/9710104 [gr-qc]]. (Cited on page 64.)

[295] Sriramkumar, L. and Shankaranarayanan, S., "Path integral duality and Planck scale corrections to the primordial spectrum in exponential inflation", J. High Energy Phys., 2006(12), 050 (2006). [DOI], [arXiv:hep-th/0608224 [hep-th]]. (Cited on page 64.)

[296] Stetsko, M.M., "Harmonic oscillator with minimal length uncertainty relations and ladder operators", Phys. Rev. A, 74, 062105 (2006). [DOI], [arXiv:quant-ph/0703269 [quant-ph]]. (Cited on page 59.)

[297] Susskind, L., "String theory and the principles of black hole complementarity", Phys. Rev. Lett., 71, 2367-2368 (1993). [DOI], [arXiv:hep-th/9307168]. (Cited on page 23.)

[298] Susskind, L., "Strings, black holes and Lorentz contraction", Phys. Rev. D, 49, 6606-6611 (1994). [DOI], [arXiv:hep-th/9308139]. (Cited on page 23.)

[299] Szabo, R.J., "BUSSTEPP lectures on string theory: An introduction to string theory and D-brane dynamics", arXiv, e-print, (2002). [arXiv:hep-th/0207142]. (Cited on page 22.)

[300] 't Hooft, G. and Veltman, M., "One-loop divergencies in the theory of gravitation", Ann. Inst. Henri Poincare A, 20, 69-94 (1974). Online version (accessed 20 November 2012):

http://www .numdam.org/item?id=AIHPA_1974_20_1_69_0. (Cited on page 37.)

[301] Tamaki, T., Harada, T., Miyamoto, U. and Torii, T., "Have we already detected astrophysical symptoms of space-time noncommutativity?", Phys. Rev. D, 65, 083003 (2002). [DOI], [arXiv:grqc/0111056 [gr-qc]]. (Cited on pages 58 and 62.)

[302] Tezuka, K.-I., "Uncertainty of Velocity in kappa-Minkowski Spacetime", arXiv, e-print, (2003). [arXiv:hep-th/0302126 [hep-th]]. (Cited on page 57.)

[303] Thiemann, T., "Closed formula for the matrix elements of the volume operator in canonical quantum gravity", J. Math. Phys., 39, 3347-3371 (1998). [DOI], [arXiv:gr-qc/9606091]. (Cited on page 34.)

[304] Thiemann, T., "Loop quantum gravity: An inside view", in Stamatescu, I.-O. and Seiler, E., eds., Approaches to Fundamental Physics: An Assessment of Current Theoretical Ideas, Lecture Notes in Physics, 721, pp. 185-263, (Springer, Berlin; New York, 2007). [DOI], [arXiv:hep-th/0608210]. (Cited on page 32.)

[305] Thiemann, T., Modern Canonical Quantum General Relativity, Cambridge Monographs on Mathematical Physics, (Cambridge University Press, Cambridge; New York, 2007). [arXiv:gr-qc/0110034]. (Cited on page 32.)

[306] Thorne, K.S., "Nonspherical gravitational collapse: A short review", in Klauder, J.R., ed., Magic Without Magic: John Archibald Wheeler. A Collection of Essays in Honor of his Sixtieth Birthday, pp. 231-258, (W.H. Freeman, San Francisco, 1972). [ADS]. (Cited on page 13.)

[307] Tomassini, L. and Viaggiu, S., "Physically motivated uncertainty relations at the Planck length for an emergent non-commutative spacetime", Class. Quantum Grav., 28, 075001 (2011). [DOI], [arXiv:1102.0894 [gr-qc]]. (Cited on page 21.)

[308] Unruh, W.G., "Sonic analog of black holes and the effects of high frequencies on black hole evaporation", Phys. Rev. D, 51, 2827-2838 (1995). [DOI], [arXiv:gr-qc/9409008]. (Cited on page 9.)

[309] Vakili, B., "Cosmology with minimal length uncertainty relations", Int. J. Mod. Phys. D, 18, 10591071 (2009). [DOI], [arXiv:0811.3481 [gr-qc]]. (Cited on page 64.) 
[310] Veneziano, G., "An enlarged uncertainty principle from gedanken string collisions?", in Arnowitt, R.L., Bryan, R. and Duff, M.J., eds., Strings '89, 3rd International Superstring Workshop, Texas A\&M University, College Station, TX, March 13-8, 1989, pp. 86-103, (World Scientific, Singapore, 1990). Online version (accessed 29 March 2012): http://cdsweb.cern.ch/record/197729/. (Cited on pages 10 and 24.)

[311] Vilela Mendes, R., "Some consequences of a non-commutative space-time structure", Eur. Phys. J. C, 42, 445-452 (2005). [DOI], [arXiv:hep-th/0406013 [hep-th]]. (Cited on page 61.)

[312] Wang, P., Yang, H. and Zhang, X., "Quantum gravity effects on compact star cores", arXiv, e-print, (2011). [arXiv:1110.5550 [gr-qc]]. (Cited on page 63.)

[313] Wess, J., "Nonabelian gauge theories on noncommutative spaces", in Nath, P., Zerwas, P.M. and Grosche, C., eds., The 10th International Conference On Supersymmetry And Unification Of Fundamental Interactions (SUSY02), June 17-23, 2002, DESY Hamburg, pp. 586-599, (DESY, Hamburg, 2002). Online version (accessed 29 March 2012): http://www-library.desy.de/preparch/desy/proc/proc02-02.html. (Cited on page 8.)

[314] Wohlgenannt, M., "Non-commutative Geometry \& Physics", Ukr. J. Phys., 55, 5-14 (2010). [arXiv:hep-th/0602105 [hep-th]]. URL (accessed 15 November 2012): http://ujp.bitp.kiev.ua/index.php?item=j\&id=122. (Cited on page 42.)

[315] Xiang, L. and Wen, X.Q., "Black hole thermodynamics with generalized uncertainty principle", $J$. High Energy Phys., 2009(10), 046 (2009). [DOI], [arXiv:0901.0603 [gr-qc]]. (Cited on page 64.)

[316] Yang, C.N., "On quantized space-time", Phys. Rev., 72, 874 (1947). [DOI]. (Cited on page 8.)

[317] Yoneya, T., "On the interpretation of minimal length in string theories", Mod. Phys. Lett. A, 4, 1587 (1989). [DOI]. (Cited on page 26.)

[318] Yoneya, T., "String theory and space-time uncertainty principle", Prog. Theor. Phys., 103, 10811125 (2000). [DOI], [arXiv:hep-th/0004074]. (Cited on pages 24, 25, 26, 27, 28, and 29.)

[319] Yoon, M., Ha, J. and Kim, W., "Entropy of Reissner-Nordstrom black holes with minimal length revisited", Phys. Rev. D, 76, 047501 (2007). [DOI], [arXiv:0706.0364 [gr-qc]]. (Cited on page 64.)

[320] Zhang, X., Shao, L. and Ma, B.-Q., "Photon Gas Thermodynamics in Doubly Special Relativity", Astropart. Phys., 34, 840-845 (2011). [DOI], [arXiv:1102.2613 [hep-th]]. (Cited on page 63.)

[321] Zhao, H.-X., Li, H.-F., Hu, S.-Q. and Zhao, R., "Generalized Uncertainty Principle and Black Hole Entropy of Higher-Dimensional de Sitter Spacetime", Commun. Theor. Phys., 48, 465-468 (2007). [DOI], [arXiv:gr-qc/0608023 [gr-qc]]. (Cited on page 64.) 